\title{
Photo-Induced Rapid Multi-Component Cascade Reaction of Aryldiazonium Salts with Unactivated Alkenes and TMSN3
}

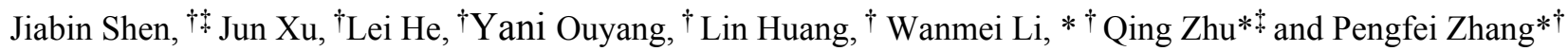

${ }^{\dagger}$ College of Material Chemistry and Chemical Engineering, Hangzhou Normal University, Hangzhou 310036, China.

$\$$ Collaborative Innovation Center of Yangtze River Delta Region Green Pharmaceuticals, Zhejiang

University of Technology, Hangzhou 310014, China.

Email: wanmeili@hznu.edu.cn (Li,W.); zhuq@zjut.edu.cn (Zhu, Q.) ; chxyzpf@hotmail.com (Zhang, P.)

\section{Supporting Information}

\section{Table of contents}

1. General Information 2

2. Experimental Section 2

3. Characterization of Products 6

4. NMR Spectra for the Products 17

5. High Resolution Mass Spectrometry 53 


\section{General Information}

All reagents and deuterated solvents were commercially available and used without further purification. All products were separated by silica gel (200-300 mesh) column chromatography with petroleum ether $(\mathrm{PE})\left(60-90{ }^{\circ} \mathrm{C}\right)$ and ethyl acetate (EA). ${ }^{1} \mathrm{H}$ and ${ }^{13} \mathrm{C}$ NMR spectra were recorded on a Bruker Advance 500 spectrometer at ambient temperature with $\mathrm{CDCl}_{3}$ as solvent and tetramethylsilane (TMS) as the internal standard. Melting points were determined on an X-5 Data microscopic melting point apparatus. Analytical thin layer chromatography (TLC) was performed on Merk precoated TLC (silica gel 60 F254) plates. Compounds for HRMS were analyzed by positive mode electrospray ionization (ESI) using Agilent 6530 QTOF mass spectrometer.

\section{Experimental Section}

\subsection{General procedure for the synthesis of diazonium tetrafluoroborates 1}

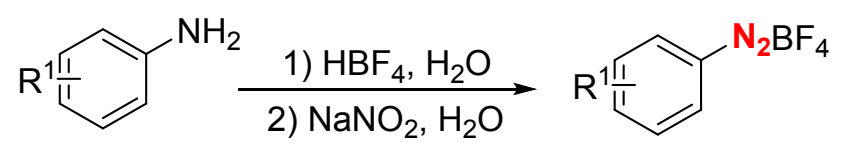

The appropriate aniline $(10.0 \mathrm{mmol})$ was dissolved in a mixture of $4.0 \mathrm{~mL}$ of distilled water and $3.4 \mathrm{~mL}$ of $50 \%$ hydrofluoroboric acid. After the reaction mixture was cooling to $0{ }^{\circ} \mathrm{C}$ using ice bath and the sodium nitrite $\left(690 \mathrm{mg}\right.$ in $\left.1.5 \mathrm{~mL} \mathrm{H}_{2} \mathrm{O}\right)$ was added dropwise in 5 min interval of time. The resulting mixture was stirred for $40 \mathrm{~min}$ and the precipitate was collected by filtration and re-dissolved in minimum amount of acetone. Diethyl ether was added until precipitation of diazonium tetrafluoroborate, it was filtered and washed with diethyl ether and dried under vacuum to provide the diazonium tetrafluoroborates 1 .

\subsection{General procedure for the synthesis of alkenes 2}

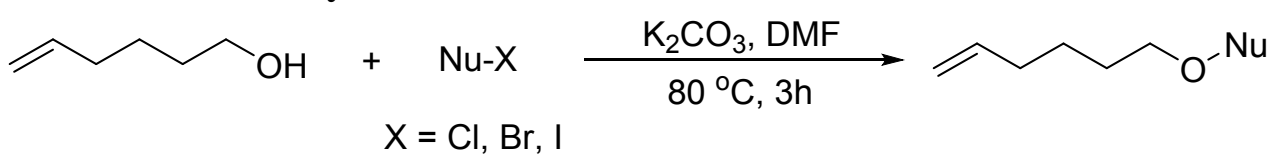

A mixture of 5-Hexen-ol (10.0 mmol), $\mathrm{K}_{2} \mathrm{CO}_{3}(11.0 \mathrm{mmol})$ and $\mathrm{DMF}(25 \mathrm{~mL})$ in a $100 \mathrm{~mL}$ tube. After the reaction mixture was cooling to $0{ }^{\circ} \mathrm{C}$ using ice bath and the appropriate acyl chloride was added dropwise in 5 min interval of time. The resulting mixture was stirred for $3 \mathrm{~h}$ at $80{ }^{\circ} \mathrm{C}$. After the completion (as indicated by TLC), the reaction mixture was quenched with saturated $\mathrm{NaHCO}_{3}$ aqueous solution, and extracted with EtOAc $(5 \mathrm{~mL} \times 3)$. The collected organic layer was washed with brine, and dried with $\mathrm{MgSO}_{4}$. Finally, the organic solvent was removed under reduced pressure, and the obtained residue was further purified by silica gel column chromatography (200-300 mesh silica gel, PE/EA = 20:1) to provide the alkenes 2.

\subsection{General procedure for the synthesis of products 4}

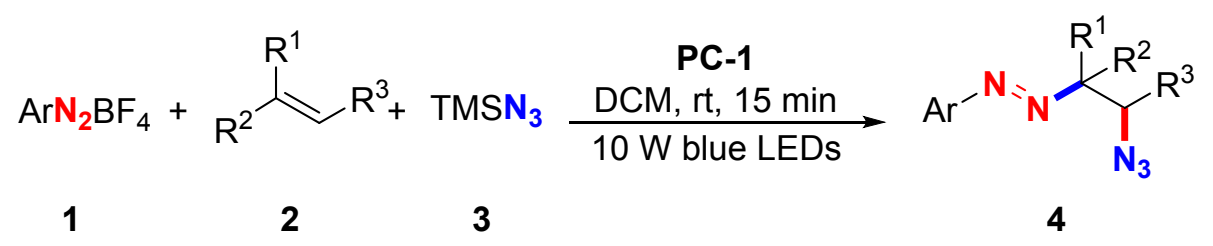

A mixture of aryl diazonium (1) (0.2 mmol), alkenes (2) (0.26 mmol), TMSN 3 (0.26 mmol), PC-1 $(2 \mathrm{~mol} \%)$ and DCM $(1.0 \mathrm{~mL})$ in a $10 \mathrm{~mL}$ tube was stirred under a $10 \mathrm{~W}$ blue LEDs at room temperature for $15 \mathrm{~min}$. After the completion (as indicated by TLC), the reaction mixture was 
quenched with saturated $\mathrm{NaHCO}_{3}$ aqueous solution, and extracted with EtOAc $(5 \mathrm{~mL} \times 3)$. The collected organic layer was washed with brine, and dried with $\mathrm{MgSO}_{4}$. Finally, the organic solvent was removed under reduced pressure, and the obtained residue was further purified by silica gel column chromatography $(200-300$ mesh silica gel, PE/EA $=11: 1)$ to provide the products 4.

\subsection{General procedure for gram-scale synthesis of product $4 d$}

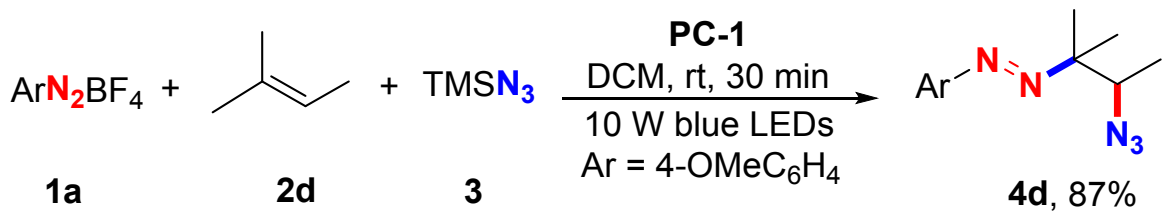

A mixture of 4-methoxybenzenediazonium tetrafluoroborate (1a) (4.0 mmol), 2-methylbut-2-ene (2d) $(8.0 \mathrm{mmol}), \mathrm{TMSN}_{3}(8.0 \mathrm{mmol}), \mathrm{PC}-1(2 \mathrm{~mol} \%)$ and DCM $(10 \mathrm{~mL})$ in a $50 \mathrm{~mL}$ round-bottom flask was stirred under a $10 \mathrm{~W}$ blue LEDs at room temperature for $30 \mathrm{~min}$. After the completion (as indicated by TLC), the reaction mixture was quenched with saturated $\mathrm{NaHCO}_{3}$ aqueous solution, and extracted with EtOAc $(20 \mathrm{~mL} \times 3)$. The collected organic layer was washed with brine, and dried with $\mathrm{MgSO}_{4}$. Finally, the organic solvent was removed under reduced pressure, and the obtained residue was further purified by silica gel column chromatography (200-300 mesh silica gel, $\mathrm{PE} / \mathrm{EA}=10: 1)$ to provide product $\mathbf{4 d}$ in $87 \%$ yield $(0.86 \mathrm{~g})$.

\section{F1. Picture of the reaction setup}

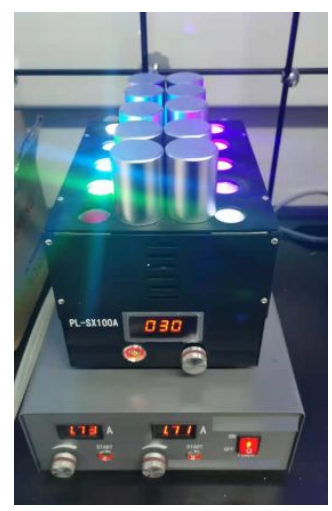

All photochemical reactions were carried out using a PL-SX100A (http://www.bjplss.com/pl/) with wavelength of peak intensity of $405-470 \mathrm{~nm}$.

\section{F2. Picture of the emission quenching experiments under different atmosphere.}

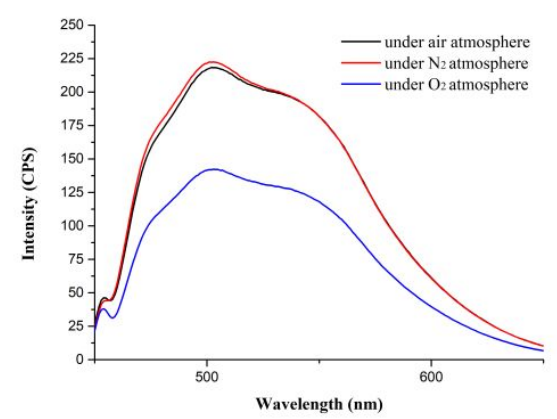

The quenching of the excited state $\mathbf{A c r}^{+}-\mathbf{M e s C l O}_{4}{ }^{-*}(0.5 \mathrm{mmol} / \mathrm{L})$ by $\mathbf{T M S N}_{3}(25 \mathrm{mmol} / \mathrm{L})$ separately was carried out in $\mathrm{CH}_{3} \mathrm{CN}\left(\lambda_{\mathrm{ex}}=445 \mathrm{~nm} ; \lambda_{\mathrm{em}}=511 \mathrm{~nm}\right)$. 


\subsection{Further chemistry}

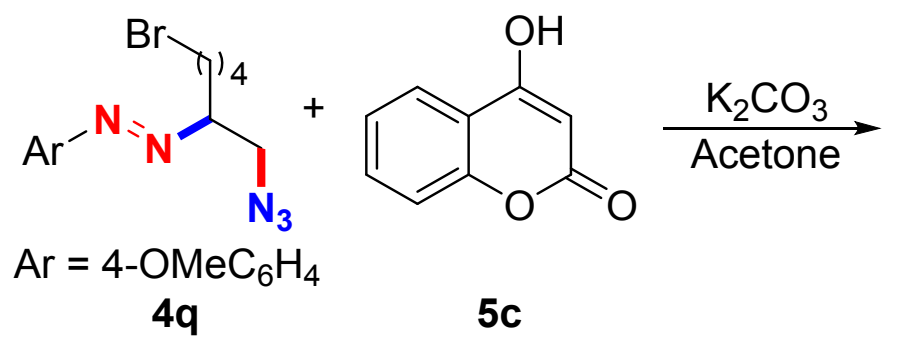<smiles>CC(C)(C)O[Mg]</smiles>

A mixture of $\mathbf{4 q}(0.2 \mathrm{mmol})$, 4-hydroxycoumarin $(\mathbf{5 c})(0.24 \mathrm{mmol}), \mathrm{K}_{2} \mathrm{CO}_{3}(0.24 \mathrm{mmol})$ and acetone $(2.0 \mathrm{~mL}$ ) in a $10 \mathrm{~mL}$ tube was stirred at room temperature for $12 \mathrm{~h}$. After the completion (as indicated by TLC), the reaction mixture was quenched with saturated $\mathrm{NaHCO}_{3}$ aqueous solution, and extracted with EtOAc $(5 \mathrm{~mL} \times 3)$. The collected organic layer was washed with brine, and dried with $\mathrm{MgSO}_{4}$. Finally, the organic solvent was removed under reduced pressure, and the obtained residue was further purified by silica gel column chromatography (200-300 mesh silica gel, PE/EA $=5: 1$ ) to provide product 4 ae in $84 \%$ yield.<smiles>C#Cc1ccccc1</smiles>

A mixture of $\mathbf{4 q}(0.2 \mathrm{mmol})$, phenylacetylene $\mathbf{5 d}(0.30 \mathrm{mmol}), \mathrm{CuI}(0.30 \mathrm{mmol})$, DIPEA $(0.60 \mathrm{mmol})$ and $\mathrm{MeCN}(2.5 \mathrm{~mL}$ ) in a $10 \mathrm{~mL}$ tube was stirred at room temperature for $3 \mathrm{~h}$. After the completion (as indicated by TLC), the reaction mixture was quenched with saturated $\mathrm{NaHCO}_{3}$ aqueous solution, and extracted with EtOAc $(5 \mathrm{~mL} \times 3)$. The collected organic layer was washed with brine, and dried with $\mathrm{MgSO}_{4}$. Finally, the organic solvent was removed under reduced pressure, and the obtained residue was further purified by silica gel column chromatography (200-300 mesh silica gel, PE/EA $=7: 1$ ) to provide product 4 af in $64 \%$ yield.<smiles>COc1ccc(N=NC2CCCCC2N=Nc2ccccc2)cc1</smiles>

A mixture of $4 \mathbf{e}(0.2 \mathrm{mmol}), \mathrm{Pd} / \mathrm{C}(10 \mathrm{wt} \%, 11.0 \mathrm{mg})$ and $\mathrm{EtOH}(2.5 \mathrm{~mL})$ in a $10 \mathrm{~mL}$ tube was degassed through three freeze-pump-thaw cycles under $\mathrm{H}_{2}$. After stirred for $9 \mathrm{~h}$ at room temperature. The reaction mixture was filtered through silica gel and condensed in vacuo to obtain the product 4ag in $81 \%$ yield.

\subsection{Modification of natural products}



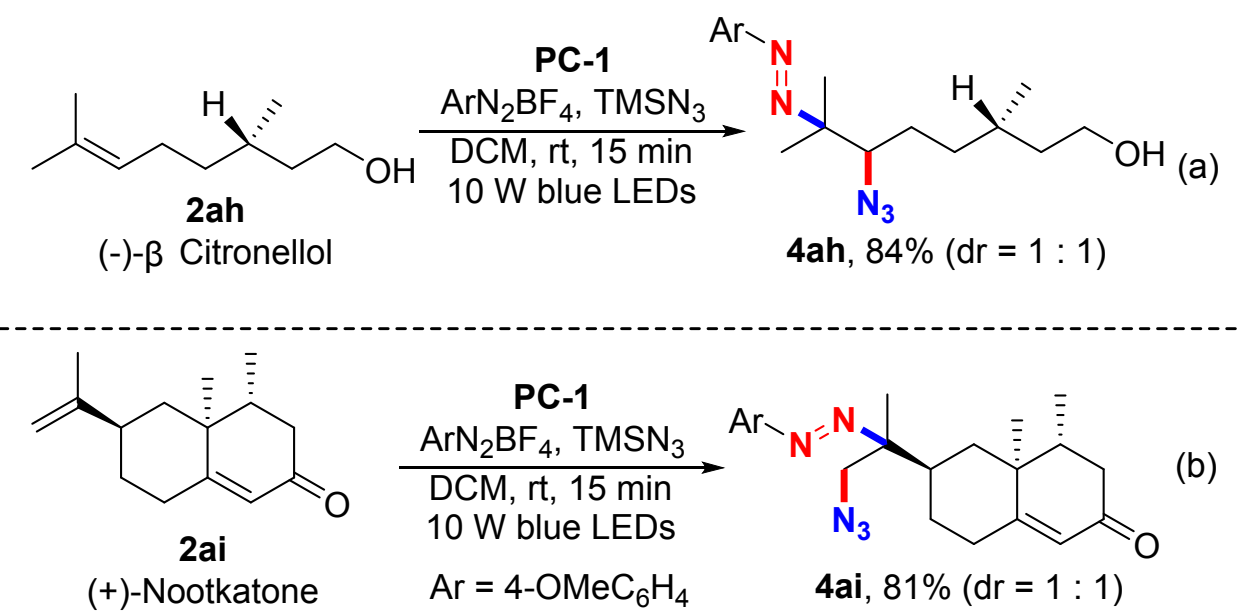

A mixture of aryl diazonium (1a) $(0.2 \mathrm{mmol}), \mathbf{2} \mathbf{a h}-\mathbf{i}(0.26 \mathrm{mmol}), \mathrm{TMSN}_{3}(0.26 \mathrm{mmol}), \mathbf{P C}-\mathbf{1}$ (2 mol \%) and DCM (1.0 mL) in a $10 \mathrm{~mL}$ tube was stirred under a $10 \mathrm{~W}$ blue LEDs at room temperature for $15 \mathrm{~min}$. After the completion (as indicated by TLC), the reaction mixture was quenched with saturated $\mathrm{NaHCO}_{3}$ aqueous solution, and extracted with EtOAc $(5 \mathrm{~mL} \times 3)$. The collected organic layer was washed with brine, and dried with $\mathrm{MgSO}_{4}$. Finally, the organic solvent was removed under reduced pressure, and the obtained residue was further purified by silica gel column chromatography $(200-300$ mesh silica gel, $\mathrm{PE} / \mathrm{EA}=7: 1)$ to provide the products $\mathbf{4 a h}-\mathbf{i}$.

\subsection{Modification of nitrogenous alkenes}
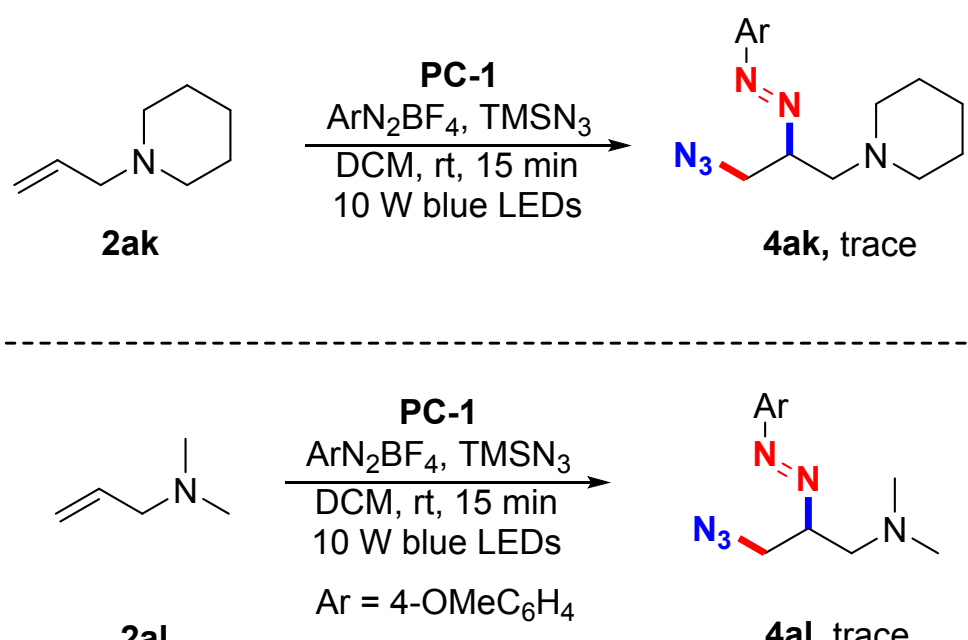<smiles></smiles>

2al

4al, trace

A mixture of aryl diazonium (1a) $(0.2 \mathrm{mmol}), \mathbf{2 a k}-\mathbf{l}(0.26 \mathrm{mmol}), \mathrm{TMSN}_{3}(0.26 \mathrm{mmol}), \mathbf{P C}-\mathbf{1}$ (2 mol \%) and DCM $(1.0 \mathrm{~mL})$ in a $10 \mathrm{~mL}$ tube was stirred under a $10 \mathrm{~W}$ blue LEDs at room temperature for $15 \mathrm{~min}$. After the completion (as indicated by TLC), no desired product was detected.

\subsection{Emission quenching experiments}

The quenching of the excited state $\mathbf{A c r}^{+}-\mathbf{M e s C l O}_{4}{ }^{-*}$ by $\mathbf{T} \mathbf{M S N}{ }_{3}$ separately was carried out in $\mathrm{CH}_{3} \mathrm{CN}$. The results revealed that $\mathbf{T M S N}_{3}$ could significantly quench $\mathbf{A c r}^{+}-\mathbf{M e s C l O}_{4}^{-*}\left(\lambda_{\mathrm{ex}}=445 \mathrm{~nm} ; \lambda_{\mathrm{em}}=511 \mathrm{~nm}\right)$. 


\section{Characterization of the products}

\section{1-(1-Azidopentan-2-yl)-2-(4-methoxyphenyl)diazene (4a)}<smiles>CCCC(CN)N=Nc1ccc(OC)cc1</smiles>

The product was obtained after purification by column chromatography (gradient hexane/EtOAc $50: 1$ to $10: 1)$ as a yellow oil (47 mg, $95 \%$ yield); ${ }^{1} \mathrm{H}$ NMR $\left(500 \mathrm{MHz}, \mathrm{CDCl}_{3}\right) \delta 7.71(\mathrm{~d}, J=8.9 \mathrm{~Hz}$, $2 \mathrm{H}), 6.96(\mathrm{~d}, J=9.0 \mathrm{~Hz}, 2 \mathrm{H}), 3.86(\mathrm{~s}, 3 \mathrm{H}), 3.82(\mathrm{~s}, 1 \mathrm{H}), 3.80-3.75(\mathrm{~m}, 1 \mathrm{H}), 3.56(\mathrm{dd}, J=12.3,3.7$ $\mathrm{Hz}, 1 \mathrm{H}), 1.94-1.87(\mathrm{~m}, 1 \mathrm{H}), 1.78-1.71(\mathrm{~m}, 1 \mathrm{H}), 1.35-1.30(\mathrm{~m}, 2 \mathrm{H}), 0.92(\mathrm{t}, J=7.3 \mathrm{~Hz}, 3 \mathrm{H}) .{ }^{13} \mathrm{C}$ NMR $\left(126 \mathrm{MHz}, \mathrm{CDCl}_{3}\right) \delta 161.8,146.1,124.26,114.1,76.4,55.6,53.7,33.1,19.1,14.0$; HRMS (ESI) $\mathrm{m} / \mathrm{z}:[\mathrm{M}+\mathrm{H}]^{+}$Calcd for $\mathrm{C}_{12} \mathrm{H}_{17} \mathrm{~N}_{5} \mathrm{OH}: 248.1506$, Found 248.1506.

\section{1-(1-Azidohexan-2-yl)-2-(4-methoxyphenyl)diazene (4b)}<smiles>CCC(CN)N=Nc1ccc(OC)cc1</smiles>

The product was obtained after purification by column chromatography (gradient hexane/EtOAc $50: 1$ to $10: 1)$ as a yellow oil (48 $\mathrm{mg}, 92 \%$ yield); ${ }^{1} \mathrm{H} \mathrm{NMR}\left(500 \mathrm{MHz}, \mathrm{CDCl}_{3}\right) \delta 7.71(\mathrm{~d}, J=8.9 \mathrm{~Hz}$, $2 \mathrm{H}), 6.96(\mathrm{~d}, J=9.0 \mathrm{~Hz}, 2 \mathrm{H}), 3.86(\mathrm{~s}, 3 \mathrm{H}), 3.82-3.75(\mathrm{~m}, 2 \mathrm{H}), 3.60-3.54(\mathrm{~m}, 1 \mathrm{H}), 1.95-1.85(\mathrm{~m}$, $1 \mathrm{H}), 1.80-1.73(\mathrm{~m}, 1 \mathrm{H}), 1.32(\mathrm{~d}, J=6.9 \mathrm{~Hz}, 2 \mathrm{H}), 1.27(\mathrm{~s}, 2 \mathrm{H}), 0.88(\mathrm{t}, J=7.1 \mathrm{~Hz}, 3 \mathrm{H}) ;{ }^{13} \mathrm{C} \mathrm{NMR}$ $\left(126 \mathrm{MHz}, \mathrm{CDCl}_{3}\right) \delta 161.8,146.1,124.3,114.1,76.7,55.6,53.7,30.7,28.0,22.6,13.9$; HRMS (ESI) $\mathrm{m} / \mathrm{z}:[\mathrm{M}+\mathrm{H}]^{+}$Calcd for $\mathrm{C}_{13} \mathrm{H}_{19} \mathrm{~N}_{5} \mathrm{OH}: 262.1663$, Found 262.1662.

\section{1-(1-Azidooctan-2-yl)-2-(4-methoxyphenyl)diazene (4c)}<smiles>CCC(CN)N=Nc1ccc(OC)cc1</smiles>

The product was obtained after purification by column chromatography (gradient hexane/EtOAc $50: 1$ to $10: 1)$ as a yellow oil $\left(55 \mathrm{mg}, 95 \%\right.$ yield); ${ }^{1} \mathrm{H}$ NMR $\left(500 \mathrm{MHz}, \mathrm{CDCl}_{3}\right) \delta 7.64(\mathrm{~d}, J=8.5 \mathrm{~Hz}$, $2 \mathrm{H}), 6.88(\mathrm{~d}, J=8.6 \mathrm{~Hz}, 2 \mathrm{H}), 3.78(\mathrm{~s}, 3 \mathrm{H}), 3.74-3.67(\mathrm{~m}, 2 \mathrm{H}), 3.52-3.46(\mathrm{~m}, 1 \mathrm{H}), 1.86-1.78(\mathrm{~m}$, $1 \mathrm{H}), 1.68(\mathrm{dd}, J=10.1,4.6 \mathrm{~Hz}, 1 \mathrm{H}), 1.24-1.17(\mathrm{~m}, 8 \mathrm{H}), 0.79(\mathrm{t}, J=6.7 \mathrm{~Hz}, 3 \mathrm{H}) ;{ }^{13} \mathrm{C}$ NMR $(126$ $\left.\mathrm{MHz}, \mathrm{CDCl}_{3}\right) \delta 161.8,146.1,124.3,114.1,76.7,55.5,53.7,31.6,31.0,29.2,25.8,22.6,14.0$; HRMS (ESI) m/z: [M+Na] $]^{+}$Calcd for $\mathrm{C}_{15} \mathrm{H}_{23} \mathrm{~N}_{5} \mathrm{ONa}$ : 312.1795, Found 312.1790.

\section{1-(3-Azido-2-methylbutan-2-yl)-2-(4-methoxyphenyl)diazene (4d)}<smiles>COc1ccc(/N=N/C(C)(C)C(C)N)cc1</smiles>

The product was obtained after purification by column chromatography (gradient hexane/EtOAc 50:1 to $10: 1)$ as a yellow oil (46 mg, 93\% yield); ${ }^{1} \mathrm{H}$ NMR $\left(500 \mathrm{MHz}, \mathrm{CDCl}_{3}\right) \delta 7.70(\mathrm{~d}, J=8.8 \mathrm{~Hz}$, 2H), 6.95 (d, $J=8.8 \mathrm{~Hz}, 2 \mathrm{H}), 3.95$ (q, $J=6.7 \mathrm{~Hz}, 1 \mathrm{H}), 3.86(\mathrm{~s}, 3 \mathrm{H}), 1.33$ (d, $J=6.7 \mathrm{~Hz}, 3 \mathrm{H}), 1.26$ (s, 3H), $1.23(\mathrm{~s}, 3 \mathrm{H}) ;{ }^{13} \mathrm{C}$ NMR $\left(126 \mathrm{MHz}, \mathrm{CDCl}_{3}\right) \delta 161.6,146.2,124.1,114.0,72.8,64.8,55.6$, 21.8, 21.3, 14.6; HRMS (ESI) m/z: [M+Na] ${ }^{+}$Calcd for $\mathrm{C}_{12} \mathrm{H}_{17} \mathrm{~N}_{5} \mathrm{ONa}$ : 270.1325, Found 270.1325. 
<smiles>COc1ccc(/N=N/C2CCCCC2N)cc1</smiles>

The product was obtained after purification by column chromatography (gradient hexane/EtOAc $50: 1$ to $10: 1)$ as a yellow oil, $1: 1 \mathrm{dr}(38 \mathrm{mg}, 73 \%$ yield $) ;{ }^{1} \mathrm{H} \mathrm{NMR}\left(500 \mathrm{MHz}, \mathrm{CDCl}_{3}\right) \delta 7.72(\mathrm{t}, J=$ $9.1 \mathrm{~Hz}, 2 \mathrm{H}), 6.98-6.93(\mathrm{~m}, 2 \mathrm{H}), 4.01(\mathrm{dd}, J=10.9,4.3 \mathrm{~Hz}, 1 \mathrm{H}), 3.86(\mathrm{~s}, 3 \mathrm{H}), 3.80-3.77(\mathrm{~m}$, $0.5 \mathrm{H}), 3.56-3.50(\mathrm{~m}, 0.65 \mathrm{H}), 2.23-2.14(\mathrm{~m}, 1 \mathrm{H}), 1.89-1.83(\mathrm{~m}, 2 \mathrm{H}), 1.81-1.75(\mathrm{~m}, 2 \mathrm{H}), 1.74$ $-1.55(\mathrm{~m}, 1 \mathrm{H}), 1.50-1.39(\mathrm{~m}, 2 \mathrm{H}) ;{ }^{13} \mathrm{C} \mathrm{NMR}\left(126 \mathrm{MHz}, \mathrm{CDCl}_{3}\right) \delta 161.8,146.2,124.3,114.1$, 80.0, 63.1, 55.6, 30.4, 29.8, 24.4, 23.6; HRMS (ESI) m/z: $[\mathrm{M}+\mathrm{Na}]^{+}$Calcd for $\mathrm{C}_{13} \mathrm{H}_{17} \mathrm{~N}_{5} \mathrm{ONa}$ : 282.1325 , Found 282.1328 .

1-(1-Azido-3-phenylpropan-2-yl)-2-(4-methoxyphenyl)diazene (4f)<smiles>COc1ccc(N=NC(CN)Cc2ccccc2)cc1</smiles>

The product was obtained after purification by column chromatography (gradient hexane/EtOAc 50:1 to $10: 1)$ as a yellow oil (46 mg, 78\% yield); ${ }^{1} \mathrm{H} \mathrm{NMR}\left(500 \mathrm{MHz}, \mathrm{CDCl}_{3}\right) \delta 7.63(\mathrm{~d}, J=8.9 \mathrm{~Hz}$, $2 \mathrm{H}), 7.23-7.19(\mathrm{~m}, 2 \mathrm{H}), 7.16-7.11(\mathrm{~m}, 3 \mathrm{H}), 6.88(\mathrm{~d}, J=8.9 \mathrm{~Hz}, 2 \mathrm{H}), 4.05-4.03(\mathrm{~m}, 1 \mathrm{H}), 3.79(\mathrm{~s}$, $3 \mathrm{H}), 3.64-3.62(\mathrm{~m}, 1 \mathrm{H}), 3.52-3.39(\mathrm{~m}, 1 \mathrm{H}), 3.08-3.04(\mathrm{~m}, 2 \mathrm{H}) ;{ }^{13} \mathrm{C} \mathrm{NMR}\left(126 \mathrm{MHz}, \mathrm{CDCl}_{3}\right) \delta$ 161.9, 146.1, 137.3, 129.5, 128.6, 126.6, 124.3, 114.1, 77.3, 55.6, 52.8, 37.3; HRMS (ESI) m/z: $[\mathrm{M}+\mathrm{Na}]^{+}$Calcd for $\mathrm{C}_{16} \mathrm{H}_{17} \mathrm{~N}_{5} \mathrm{ONa}$ : 318.1325, Found 318.1328.

\section{5-Azido-4-((4-methoxyphenyl)diazenyl)pentan-1-ol (4g)}<smiles>COc1ccc(N=NC(CN)CO)cc1</smiles>

The product was obtained after purification by column chromatography (gradient hexane/EtOAc 40:1 to 6:1) as a yellow oil (40 mg, 76\% yield); ${ }^{1} \mathrm{H} \mathrm{NMR}\left(500 \mathrm{MHz}, \mathrm{CDCl}_{3}\right) \delta 7.71(\mathrm{~d}, J=9.0 \mathrm{~Hz}$, $2 \mathrm{H}), 6.96(\mathrm{~d}, J=9.0 \mathrm{~Hz}, 2 \mathrm{H}), 3.87(\mathrm{~s}, 4 \mathrm{H}), 3.82-3.76(\mathrm{~m}, 1 \mathrm{H}), 3.66(\mathrm{td}, J=6.4,1.6 \mathrm{~Hz}, 2 \mathrm{H}), 3.60$ $(\mathrm{dd}, J=12.5,4.0 \mathrm{~Hz}, 1 \mathrm{H}), 2.05-1.97(\mathrm{~m}, 1 \mathrm{H}), 1.89(\mathrm{~d}, J=6.6 \mathrm{~Hz}, 1 \mathrm{H}), 1.62-1.55(\mathrm{~m}, 2 \mathrm{H}) ;{ }^{13} \mathrm{C}$ NMR $\left(126 \mathrm{MHz}, \mathrm{CDCl}_{3}\right) \delta 162.0,146.0,124.3,114.1,76.1,62.5,55.6,53.7,28.9,27.4$; HRMS (ESI) $\mathrm{m} / \mathrm{z}:[\mathrm{M}+\mathrm{Na}]^{+}$Calcd for $\mathrm{C}_{12} \mathrm{H}_{17} \mathrm{~N}_{5} \mathrm{O}_{2} \mathrm{Na}$ : 286.1274, Found 286.1285.

\section{6-Azido-5-((4-methoxyphenyl)diazenyl)hexan-1-ol (4h)}<smiles>COc1ccc(/N=N/C(CN)CO)cc1</smiles>

The product was obtained after purification by column chromatography (gradient hexane/EtOAc 40:1 to 6:1) as a yellow oil (43 $\mathrm{mg}, 78 \%$ yield); ${ }^{1} \mathrm{H} \mathrm{NMR}\left(500 \mathrm{MHz}, \mathrm{CDCl}_{3}\right) \delta 7.71(\mathrm{~d}, J=8.9 \mathrm{~Hz}$, $2 \mathrm{H}), 6.96(\mathrm{~d}, J=8.8 \mathrm{~Hz}, 2 \mathrm{H}), 3.86(\mathrm{~s}, 3 \mathrm{H}), 3.83(\mathrm{~s}, 1 \mathrm{H}), 3.78(\mathrm{~d}, J=12.3 \mathrm{~Hz}, 1 \mathrm{H}), 3.63(\mathrm{t}, J=6.5$ $\mathrm{Hz}, 2 \mathrm{H}), 3.59$ (d, $J=3.8 \mathrm{~Hz}, 1 \mathrm{H}), 2.02-1.91(\mathrm{~m}, 1 \mathrm{H}), 1.84-1.76(\mathrm{~m}, 1 \mathrm{H}), 1.64-1.54(\mathrm{~m}, 3 \mathrm{H})$, $1.41-1.34(\mathrm{~m}, 2 \mathrm{H}) ;{ }^{13} \mathrm{C} \mathrm{NMR}\left(126 \mathrm{MHz}, \mathrm{CDCl}_{3}\right) \delta 161.9,146.0,124.3,114.1,76.5,62.6,55.6$, 
53.7, 32.5, 30.8, 22.1; HRMS (ESI) $\mathrm{m} / \mathrm{z}$ : $[\mathrm{M}+\mathrm{Na}]^{+}$Calcd for $\mathrm{C}_{13} \mathrm{H}_{19} \mathrm{~N}_{5} \mathrm{O}_{2} \mathrm{Na}$ : 300.1431, Found 300.1429 .

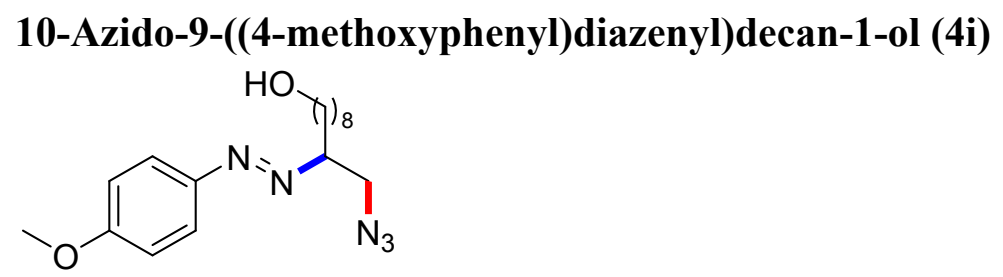

The product was obtained after purification by column chromatography (gradient hexane/EtOAc $40: 1$ to $6: 1)$ as a yellow oil $\left(57 \mathrm{mg}, 86 \%\right.$ yield); ${ }^{1} \mathrm{H} \mathrm{NMR}\left(500 \mathrm{MHz}, \mathrm{CDCl}_{3}\right) \delta 7.71(\mathrm{~d}, J=9.0 \mathrm{~Hz}$, $2 \mathrm{H}), 6.96(\mathrm{~d}, J=8.9 \mathrm{~Hz}, 2 \mathrm{H}), 3.86(\mathrm{~s}, 3 \mathrm{H}), 3.81(\mathrm{dd}, J=4.9,3.3 \mathrm{~Hz}, 1 \mathrm{H}), 3.77(\mathrm{~d}, J=12.0 \mathrm{~Hz}, 1 \mathrm{H})$, $3.62(\mathrm{t}, J=6.6 \mathrm{~Hz}, 2 \mathrm{H}), 3.57(\mathrm{~s}, 1 \mathrm{H}), 1.92-1.86(\mathrm{~m}, 1 \mathrm{H}), 1.78-1.73(\mathrm{~m}, 1 \mathrm{H}), 1.55-1.52(\mathrm{~m}, 3 \mathrm{H})$, $1.33-1.25(\mathrm{~m}, 9 \mathrm{H}) ;{ }^{13} \mathrm{C}$ NMR $\left(126 \mathrm{MHz}, \mathrm{CDCl}_{3}\right) \delta 161.8,146.1,124.3,114.1,76.6,63.0,55.6$, 53.7, 32.7, 31.0, 29.4, 29.3, 29.3, 25.8, 25.7; HRMS (ESI) m/z: [M+Na $]^{+}$Calcd for $\mathrm{C}_{17} \mathrm{H}_{27} \mathrm{~N}_{5} \mathrm{O}_{2} \mathrm{Na}$ : 356.2057, Found 356.2056.

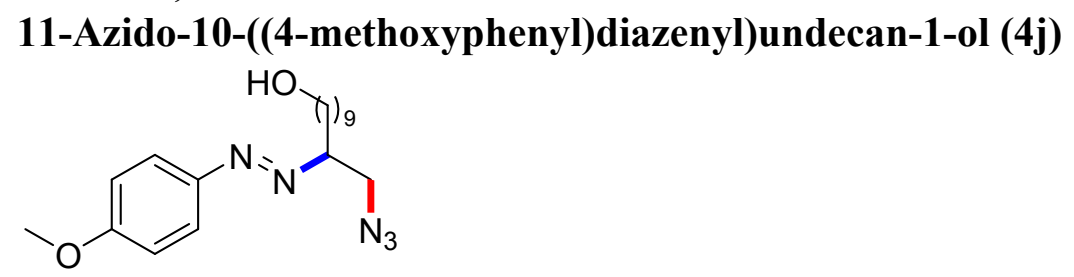

The product was obtained after purification by column chromatography (gradient hexane/EtOAc $40: 1$ to $6: 1)$ as a yellow oil $(65 \mathrm{mg}, 94 \%$ yield $) ;{ }^{1} \mathrm{H} \mathrm{NMR}\left(500 \mathrm{MHz}, \mathrm{CDCl}_{3}\right) \delta 7.70(\mathrm{~d}, J=9.0 \mathrm{~Hz}$, $2 \mathrm{H}), 6.96(\mathrm{~d}, J=9.0 \mathrm{~Hz}, 2 \mathrm{H}), 3.86(\mathrm{~s}, 3 \mathrm{H}), 3.83-3.79(\mathrm{~m}, 1 \mathrm{H}), 3.78-3.74(\mathrm{~m}, 1 \mathrm{H}), 3.62(\mathrm{t}, J=6.7$ $\mathrm{Hz}, 2 \mathrm{H}), 3.56(\mathrm{dd}, J=12.0,3.3 \mathrm{~Hz}, 1 \mathrm{H}), 1.93-1.85(\mathrm{~m}, 1 \mathrm{H}), 1.77-1.73(\mathrm{~m}, 1 \mathrm{H}), 1.56-1.50(\mathrm{~m}$, $4 \mathrm{H}), 1.32-1.26(\mathrm{~m}, 10 \mathrm{H}) ;{ }^{13} \mathrm{C} \mathrm{NMR}\left(126 \mathrm{MHz}, \mathrm{CDCl}_{3}\right) \delta 161.8,146.1,124.3,114.1,76.6,63.1$, 55.6, 53.7, 32.8, 31.0, 29.4, 29.4, 29.3, 29.3, 25.8, 25.7; HRMS (ESI) m/z: $[\mathrm{M}+\mathrm{Na}]^{+}$Calcd for $\mathrm{C}_{18} \mathrm{H}_{29} \mathrm{~N}_{5} \mathrm{O}_{2} \mathrm{Na}: 370.2213$, Found 370.2220.

\section{6-Azido-5-((4-methoxyphenyl)diazenyl)hexyl benzoate (4k)}<smiles>COc1ccc(/N=N/C(CN)COC(=O)c2ccccc2)cc1</smiles>

The product was obtained after purification by column chromatography (gradient hexane/EtOAc $20: 1$ to $3: 1)$ as a yellow oil $(67 \mathrm{mg}, 88 \%$ yield $) ;{ }^{1} \mathrm{H}$ NMR $\left(500 \mathrm{MHz}, \mathrm{CDCl}_{3}\right) \delta 8.03-7.97(\mathrm{~m}, 2 \mathrm{H})$, $7.76-7.66(\mathrm{~m}, 2 \mathrm{H}), 7.56-7.52(\mathrm{~m}, 1 \mathrm{H}), 7.40(\mathrm{t}, J=7.8 \mathrm{~Hz}, 2 \mathrm{H}), 6.99-6.91(\mathrm{~m}, 2 \mathrm{H}), 4.30(\mathrm{dt}, J=$ $6.5,3.2 \mathrm{~Hz}, 2 \mathrm{H}), 3.86-3.84(\mathrm{~m}, 4 \mathrm{H}), 3.80-3.75(\mathrm{~m}, 1 \mathrm{H}), 3.58(\mathrm{dd}, J=12.5,3.9 \mathrm{~Hz}, 1 \mathrm{H}), 2.06-$ $1.97(\mathrm{~m}, 1 \mathrm{H}), 1.87-1.75(\mathrm{~m}, 3 \mathrm{H}), 1.49-1.43(\mathrm{~m}, 2 \mathrm{H}) ;{ }^{13} \mathrm{C}$ NMR $\left(126 \mathrm{MHz}, \mathrm{CDCl}_{3}\right) \delta 166.6$, 161.9, 146.0, 132.8, 130.3, 129.5, 128.3, 124.3, 114.1, 76.3, 64.6, 55.6, 53.7, 30.6, 28.5, 22.4; HRMS (ESI) m/z: [M+Na] ${ }^{+}$Calcd for $\mathrm{C}_{20} \mathrm{H}_{23} \mathrm{~N}_{5} \mathrm{O}_{3} \mathrm{Na}$ : 404.1693, Found 404.1703.

\section{6-Azido-5-((4-methoxyphenyl)diazenyl)hexyl-adamantane-1-carboxylate (4I)}


<smiles>COc1ccc(N=NC(CN)COC(=O)C23CC4CC(CC(C4)C2)C3)cc1</smiles>

The product was obtained after purification by column chromatography (gradient hexane/EtOAc $20: 1$ to $3: 1)$ as a yellow oil $\left(71 \mathrm{mg}, 81 \%\right.$ yield); ${ }^{1} \mathrm{H}$ NMR $\left(500 \mathrm{MHz}, \mathrm{CDCl}_{3}\right) \delta 7.74-7.67(\mathrm{~m}, 2 \mathrm{H})$, $6.97-6.94(\mathrm{~m}, 2 \mathrm{H}), 4.02(\mathrm{td}, J=6.4,1.2 \mathrm{~Hz}, 2 \mathrm{H}), 3.86(\mathrm{~s}, 3 \mathrm{H}), 3.81(\mathrm{~s}, 1 \mathrm{H}), 3.78(\mathrm{~d}, J=12.2 \mathrm{~Hz}$, $1 \mathrm{H}), 3.60-3.55(\mathrm{~m}, 1 \mathrm{H}), 1.98-1.94(\mathrm{~m}, 3 \mathrm{H}), 1.82(\mathrm{~d}, J=2.9 \mathrm{~Hz}, 6 \mathrm{H}), 1.72-1.62(\mathrm{~m}, 10 \mathrm{H}), 1.36$ $(\mathrm{d}, J=7.9 \mathrm{~Hz}, 2 \mathrm{H}) ;{ }^{13} \mathrm{C} \mathrm{NMR}\left(126 \mathrm{MHz}, \mathrm{CDCl}_{3}\right) \delta 177.7,161.9,146.0,124.3,114.1,76.3,63.6$, 55.6, 53.7, 40.7, 38.9, 38.8, 36.5, 30.6, 28.5, 27.9, 22.3; HRMS (ESI) m/z: $[\mathrm{M}+\mathrm{Na}]^{+}$Calcd for $\mathrm{C}_{24} \mathrm{H}_{33} \mathrm{~N}_{5} \mathrm{O}_{3} \mathrm{Na}: 462.2476$, Found 462.2469 .

\section{6-Azido-5-((4-methoxyphenyl)diazenyl)hexyl cyclobutanecarboxylate $(4 \mathrm{~m})$}<smiles>COc1ccc(N=NC(CN)COC(=O)C2CCC2)cc1</smiles>

The product was obtained after purification by column chromatography (gradient hexane/EtOAc $20: 1$ to $3: 1)$ as a yellow oil $\left(61 \mathrm{mg}, 85 \%\right.$ yield); ${ }^{1} \mathrm{H}$ NMR $\left(500 \mathrm{MHz}, \mathrm{CDCl}_{3}\right) \delta 7.74-7.68(\mathrm{~m}, 2 \mathrm{H})$, $6.98-6.94(\mathrm{~m}, 2 \mathrm{H}), 4.04(\mathrm{td}, J=6.6,2.0 \mathrm{~Hz}, 2 \mathrm{H}), 3.87(\mathrm{~s}, 3 \mathrm{H}), 3.84-3.80(\mathrm{~m}, 1 \mathrm{H}), 3.76(\mathrm{dd}, J=$ $12.2,7.7 \mathrm{~Hz}, 1 \mathrm{H}), 3.57(\mathrm{dd}, J=12.3,3.7 \mathrm{~Hz}, 1 \mathrm{H}), 3.08(\mathrm{td}, J=8.5,1.1 \mathrm{~Hz}, 1 \mathrm{H}), 2.28-2.20$ (m, $2 \mathrm{H}), 2.18-2.11(\mathrm{~m}, 2 \mathrm{H}), 2.00-1.91(\mathrm{~m}, 2 \mathrm{H}), 1.88-1.75(\mathrm{~m}, 2 \mathrm{H}), 1.68-1.63(\mathrm{~m}, 2 \mathrm{H}), 1.36(\mathrm{t}, J=$ $7.7 \mathrm{~Hz}, 2 \mathrm{H}) ;{ }^{13} \mathrm{C} \mathrm{NMR}\left(126 \mathrm{MHz}, \mathrm{CDCl}_{3}\right) \delta 175.5,161.9,146.0,124.3,114.1,76.3,63 ., 55.6,53.7$, 38.1, 30.6, 28.5, 25.3, 22.3, 18.4; HRMS (ESI) m/z: $[\mathrm{M}+\mathrm{Na}]^{+}$Calcd for $\mathrm{C}_{18} \mathrm{H}_{25} \mathrm{~N}_{5} \mathrm{O}_{3} \mathrm{Na}: 382.1850$, Found 382.1845 .

\section{1-Azido-10-((4-methoxyphenyl)diazenyl)undecyl benzoate (4n)}<smiles>COc1ccc(/N=N/C(CN)COC(=O)c2ccccc2)cc1</smiles>

The product was obtained after purification by column chromatography (gradient hexane/EtOAc $20: 1$ to $3: 1)$ as a yellow oil (84 $\mathrm{mg}, 93 \%$ yield); ${ }^{1} \mathrm{H} \mathrm{NMR}\left(500 \mathrm{MHz}, \mathrm{CDCl}_{3}\right) \delta 7.97(\mathrm{~d}, J=7.2 \mathrm{~Hz}$, 2H), $7.64(\mathrm{~d}, J=8.9 \mathrm{~Hz}, 2 \mathrm{H}), 7.48$ (t, $J=7.4 \mathrm{~Hz}, 1 \mathrm{H}), 7.37(\mathrm{~d}, J=7.6 \mathrm{~Hz}, 2 \mathrm{H}), 6.89$ (d, $J=8.9 \mathrm{~Hz}$, 2H), $4.23(\mathrm{t}, J=6.7 \mathrm{~Hz}, 2 \mathrm{H}), 3.79(\mathrm{~s}, 3 \mathrm{H}), 3.74(\mathrm{t}, J=4.1 \mathrm{~Hz}, 1 \mathrm{H}), 3.70(\mathrm{~d}, J=12.0 \mathrm{~Hz}, 1 \mathrm{H}), 3.49$ $(\mathrm{dd}, J=12.0,3.3 \mathrm{~Hz}, 1 \mathrm{H}), 1.85-1.79(\mathrm{~m}, 1 \mathrm{H}), 1.70-1.65(\mathrm{~m}, 3 \mathrm{H}), 1.37-1.31(\mathrm{~m}, 3 \mathrm{H}), 1.26-$ $1.20(\mathrm{~m}, 9 \mathrm{H}) ;{ }^{13} \mathrm{C} \mathrm{NMR}\left(126 \mathrm{MHz}, \mathrm{CDCl}_{3}\right) \delta 166.7,161.8,146.1,132.8,130.5,129.5,128.3,124.3$, 114.1, 76.6, 65.1, 55.6, 53.7, 31.0, 29.4, 29.4, 29.3, 29.2, 28.7, 26.0, 25.8; HRMS (ESI) m/z: $[\mathrm{M}+\mathrm{Na}]^{+}$Calcd for $\mathrm{C}_{25} \mathrm{H}_{33} \mathrm{~N}_{5} \mathrm{O}_{3} \mathrm{Na}: 474.2476$, Found 474.2477. 


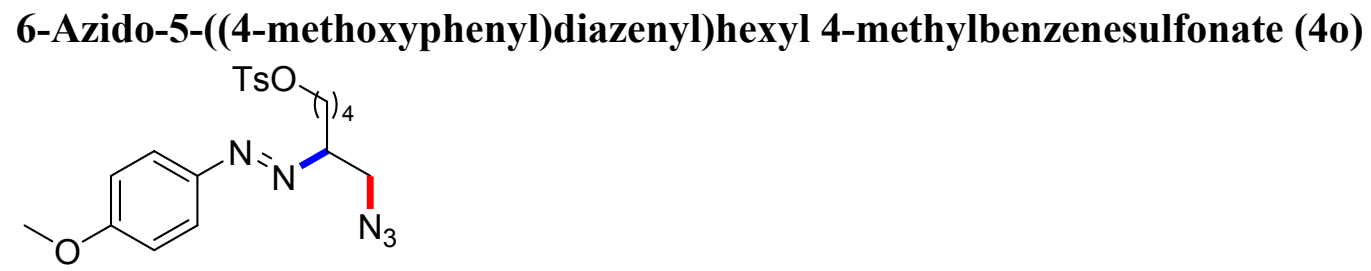

The product was obtained after purification by column chromatography (gradient hexane/EtOAc $20: 1$ to $3: 1)$ as a yellow oil (65 mg, $75 \%$ yield); ${ }^{1} \mathrm{H}$ NMR $\left(500 \mathrm{MHz}, \mathrm{CDCl}_{3}\right) \delta 7.68(\mathrm{~d}, J=8.3 \mathrm{~Hz}$, 2H), $7.62(\mathrm{~d}, J=8.9 \mathrm{~Hz}, 2 \mathrm{H}), 7.23(\mathrm{~d}, J=8.0 \mathrm{~Hz}, 2 \mathrm{H}), 6.89$ (d, $J=9.0 \mathrm{~Hz}, 2 \mathrm{H}), 3.93(\mathrm{t}, J=6.4 \mathrm{~Hz}$, $2 \mathrm{H}), 3.79(\mathrm{~s}, 3 \mathrm{H}), 3.65(\mathrm{~s}, 2 \mathrm{H}), 3.49-3.42(\mathrm{~m}, 1 \mathrm{H}), 2.36(\mathrm{~s}, 3 \mathrm{H}), 1.83-1.76(\mathrm{~m}, 1 \mathrm{H}), 1.66-1.55$ $(\mathrm{m}, 3 \mathrm{H}), 1.27-1.21(\mathrm{~m}, 2 \mathrm{H}) ;{ }^{13} \mathrm{C}$ NMR $\left(126 \mathrm{MHz}, \mathrm{CDCl}_{3}\right) \delta 162.0,146.0,144.7,133.1,129.8$, 127.9, 124.3, 114.1, 76.1, 70.1, 55.6, 53.6, 30.3, 28.7, 21.8, 21.6; HRMS (ESI) m/z: $[\mathrm{M}+\mathrm{Na}]^{+} \mathrm{Calcd}$ for $\mathrm{C}_{20} \mathrm{H}_{25} \mathrm{~N}_{5} \mathrm{O}_{4} \mathrm{SNa}$ : 454.1519, Found 454.1518 .

1-(1-Azido-6-methoxyhexan-2-yl)-2-(4-methoxyphenyl)diazene (4p)

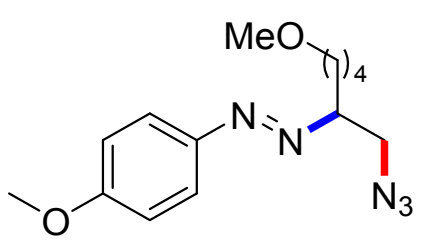

The product was obtained after purification by column chromatography (gradient hexane/EtOAc $50: 1$ to $10: 1)$ as a yellow oil $\left(50 \mathrm{mg}, 86 \%\right.$ yield); ${ }^{1} \mathrm{H}$ NMR $\left(500 \mathrm{MHz}, \mathrm{CDCl}_{3}\right) \delta 7.71(\mathrm{~d}, J=8.9 \mathrm{~Hz}$, $2 \mathrm{H}), 6.96(\mathrm{~d}, J=9.0 \mathrm{~Hz}, 2 \mathrm{H}), 3.86(\mathrm{~s}, 3 \mathrm{H}), 3.81(\mathrm{~s}, 1 \mathrm{H}), 3.78-3.74(\mathrm{~m}, 1 \mathrm{H}), 3.59-3.54(\mathrm{~m}, 1 \mathrm{H})$, $3.34(\mathrm{t}, J=6.5 \mathrm{~Hz}, 2 \mathrm{H}), 3.30(\mathrm{~s}, 3 \mathrm{H}), 1.97-1.89(\mathrm{~m}, 1 \mathrm{H}), 1.80-1.76(\mathrm{~m}, 1 \mathrm{H}), 1.61-1.56(\mathrm{~m}, 2 \mathrm{H})$, $1.36(\mathrm{dd}, J=8.9,6.9 \mathrm{~Hz}, 2 \mathrm{H}) ;{ }^{13} \mathrm{C} \mathrm{NMR}\left(126 \mathrm{MHz}, \mathrm{CDCl}_{3}\right) \delta 161.8,146.1,124.3,114.1,76.5,72.5$, 58.5, 55.6, 53.7, 30.9, 29.5, 22.6; HRMS (ESI) m/z: [M+Na $]^{+}$Calcd for $\mathrm{C}_{14} \mathrm{H}_{21} \mathrm{~N}_{5} \mathrm{O}_{2} \mathrm{Na}: 314.1587$, Found 314.1582 .

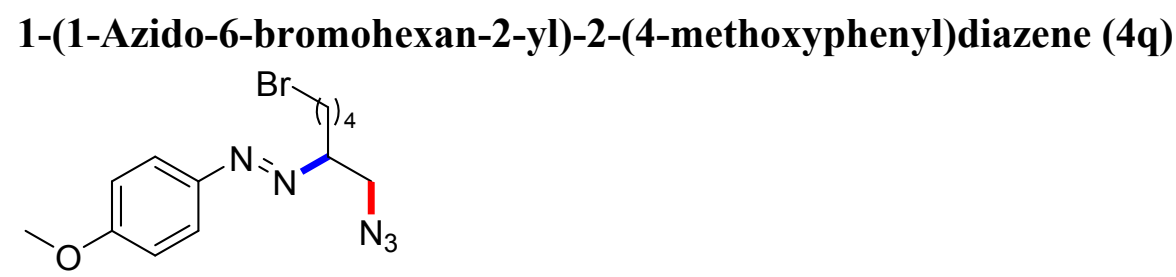

The product was obtained after purification by column chromatography (gradient hexane/EtOAc $50: 1$ to $10: 1)$ as a yellow oil $\left(59 \mathrm{mg}, 87 \%\right.$ yield); ${ }^{1} \mathrm{H}$ NMR $\left(500 \mathrm{MHz}, \mathrm{CDCl}_{3}\right) \delta 7.71(\mathrm{~d}, J=8.9 \mathrm{~Hz}$, $2 \mathrm{H}), 6.96(\mathrm{~d}, J=8.9 \mathrm{~Hz}, 2 \mathrm{H}), 3.86(\mathrm{~s}, 3 \mathrm{H}), 3.83-3.80(\mathrm{~m}, 1 \mathrm{H}), 3.78-3.75(\mathrm{~m}, 1 \mathrm{H}), 3.58(\mathrm{dd}, J=$ $12.4,3.8 \mathrm{~Hz}, 1 \mathrm{H}), 3.38(\mathrm{t}, J=6.8 \mathrm{~Hz}, 2 \mathrm{H}), 1.94(\mathrm{~d}, J=6.8 \mathrm{~Hz}, 1 \mathrm{H}), 1.91-1.85(\mathrm{~m}, 2 \mathrm{H}), 1.82-$ $1.77(\mathrm{~m}, 1 \mathrm{H}), 1.47-1.42(\mathrm{~m}, 2 \mathrm{H}) ;{ }^{13} \mathrm{C} \mathrm{NMR}\left(126 \mathrm{MHz}, \mathrm{CDCl}_{3}\right) \delta 161.9,146.0,124.3,114.1,76.2$, 55.6, 53.6, 33.3, 32.5, 30.1, 24.5; HRMS (ESI) m/z: $[\mathrm{M}+\mathrm{Na}]^{+}$Calcd for $\mathrm{C}_{13} \mathrm{H}_{18} \mathrm{BrN}_{5} \mathrm{ONa}$ : 362.0587, Found 362.0592 .

\section{1-(1-Azido-7-bromoheptan-2-yl)-2-(4-methoxyphenyl)diazene (4r)}

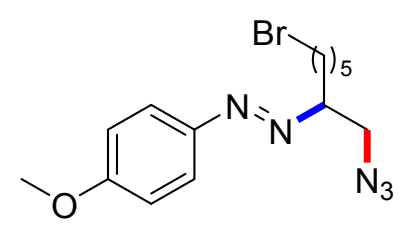

The product was obtained after purification by column chromatography (gradient hexane/EtOAc $50: 1$ to $10: 1)$ as a yellow oil $(59 \mathrm{mg}, 84 \%$ yield $) ;{ }^{1} \mathrm{H}$ NMR $\left(500 \mathrm{MHz}, \mathrm{CDCl}_{3}\right) \delta 7.71(\mathrm{~d}, J=8.9 \mathrm{~Hz}$, $2 \mathrm{H}), 6.96(\mathrm{~d}, J=8.9 \mathrm{~Hz}, 2 \mathrm{H}), 3.86(\mathrm{~s}, 3 \mathrm{H}), 3.81(\mathrm{~s}, 1 \mathrm{H}), 3.77(\mathrm{~d}, J=12.2 \mathrm{~Hz}, 1 \mathrm{H}), 3.59-3.54(\mathrm{~m}$, $1 \mathrm{H}), 3.37(\mathrm{t}, J=6.8 \mathrm{~Hz}, 2 \mathrm{H}), 1.97-1.93(\mathrm{~m}, 1 \mathrm{H}), 1.86-1.82(\mathrm{~m}, 2 \mathrm{H}), 1.79-1.77(\mathrm{~m}, 1 \mathrm{H}), 1.46$ 
- $1.42(\mathrm{~m}, 2 \mathrm{H}), 1.34-1.30(\mathrm{~m}, 2 \mathrm{H}) ;{ }^{13} \mathrm{C} \mathrm{NMR}\left(126 \mathrm{MHz}, \mathrm{CDCl}_{3}\right) \delta 161.9,146.0,124.3,114.1$, 76.4, 55.6, 53.7, 33.7, 32.5, 30.8, 28.0, 25.0; HRMS (ESI) m/z: $[\mathrm{M}+\mathrm{H}]^{+}$Calcd for $\mathrm{C}_{14} \mathrm{H}_{20} \mathrm{BrN}_{5} \mathrm{OH}$ : 354.0924, Found 354.0932.

\section{1-(1-Azidopentan-2-yl)-2-phenyldiazene (4s)}<smiles>CC(C)C(CN)N=Nc1ccccc1</smiles>

The product was obtained after purification by column chromatography (gradient hexane/EtOAc $50: 1$ to $10: 1)$ as a yellow oil $\left(36 \mathrm{mg}, 83 \%\right.$ yield); ${ }^{1} \mathrm{H}$ NMR $\left(500 \mathrm{MHz}, \mathrm{CDCl}_{3}\right) \delta 7.70(\mathrm{dd}, J=8.0$, $1.8 \mathrm{~Hz}, 2 \mathrm{H}), 7.50-7.43(\mathrm{~m}, 3 \mathrm{H}), 3.88(\mathrm{~d}, J=3.6 \mathrm{~Hz}, 1 \mathrm{H}), 3.84-3.78(\mathrm{~m}, 1 \mathrm{H}), 3.60$ (dd, $J=12.6$, $3.8 \mathrm{~Hz}, 1 \mathrm{H}), 1.97-1.90(\mathrm{~m}, 1 \mathrm{H}), 1.80-1.72(\mathrm{~m}, 1 \mathrm{H}), 1.36-1.30(\mathrm{~m}, 2 \mathrm{H}), 0.95-0.92(\mathrm{~m}, 3 \mathrm{H})$; ${ }^{13} \mathrm{C} \mathrm{NMR}\left(126 \mathrm{MHz}, \mathrm{CDCl}_{3}\right) \delta 151.9,130.8,129.0,122.4,76.8,53.6,33.0,19.1,13.9$; HRMS (ESI) $\mathrm{m} / \mathrm{z}:[\mathrm{M}+\mathrm{Na}]^{+}$Calcd for $\mathrm{C}_{11} \mathrm{H}_{15} \mathrm{~N}_{5} \mathrm{Na}: 240.1220$ Found 240.1221 .

\section{1-(1-Azidopentan-2-yl)-2-(p-tolyl)diazene (4t)}<smiles>CCCC(CN)N=Nc1ccc(C)cc1</smiles>

The product was obtained after purification by column chromatography (gradient hexane/EtOAc 50:1 to $10: 1)$ as a yellow oil $\left(41 \mathrm{mg}, 89 \%\right.$ yield); ${ }^{1} \mathrm{H}$ NMR $\left(500 \mathrm{MHz}, \mathrm{CDCl}_{3}\right) \delta 7.62(\mathrm{~d}, J=8.4 \mathrm{~Hz}$, $2 \mathrm{H}), 7.29-7.24(\mathrm{~m}, 2 \mathrm{H}), 3.86(\mathrm{dd}, J=4.9,3.6 \mathrm{~Hz}, 1 \mathrm{H}), 3.82-3.76(\mathrm{~m}, 1 \mathrm{H}), 3.58(\mathrm{dd}, J=12.5,3.8$ $\mathrm{Hz}, 1 \mathrm{H}), 2.40(\mathrm{~s}, 3 \mathrm{H}), 1.96-1.87(\mathrm{~m}, 1 \mathrm{H}), 1.75(\mathrm{~s}, 1 \mathrm{H}), 1.35-1.30(\mathrm{~m}, 2 \mathrm{H}), 0.93(\mathrm{t}, J=7.4 \mathrm{~Hz}$, $3 \mathrm{H}) ;{ }^{13} \mathrm{C}$ NMR $\left(126 \mathrm{MHz}, \mathrm{CDCl}_{3}\right) \delta 149.9,141.3,129.6,122.4,76.7,53.7,33.1,21.4,19.1,14.0$; HRMS (ESI) m/z: $[\mathrm{M}+\mathrm{Na}]^{+}$Calcd for $\mathrm{C}_{12} \mathrm{H}_{17} \mathrm{~N}_{5} \mathrm{Na}$ : 254.1376, Found 254.1378.

\section{4-((1-Azidopentan-2-yl)diazenyl)benzonitrile (4u)}<smiles>CCCC(CN)N=Nc1ccc(C#N)cc1</smiles>

The product was obtained after purification by column chromatography (gradient hexane/EtOAc 50:1 to $10: 1)$ as a yellow oil (38 mg, 79\% yield); ${ }^{1} \mathrm{H}$ NMR $\left(500 \mathrm{MHz}, \mathrm{CDCl}_{3}\right) \delta 7.78(\mathrm{~s}, 4 \mathrm{H}), 3.98-$ $3.92(\mathrm{~m}, 1 \mathrm{H}), 3.84(\mathrm{dd}, J=12.7,8.2 \mathrm{~Hz}, 1 \mathrm{H}), 3.63(\mathrm{dd}, J=12.7,3.7 \mathrm{~Hz}, 1 \mathrm{H}), 1.97-1.89(\mathrm{~m}, 1 \mathrm{H})$, $1.82-1.74(\mathrm{~m}, 1 \mathrm{H}), 1.35-1.29(\mathrm{~m}, 2 \mathrm{H}), 0.94(\mathrm{t}, J=7.4 \mathrm{~Hz}, 3 \mathrm{H}) ;{ }^{13} \mathrm{C} \mathrm{NMR}\left(126 \mathrm{MHz}, \mathrm{CDCl}_{3}\right) \delta$ 153.6, 133.2, 123.0, 118.3, 114.2, 77.5, 53.4, 32.9, 19.1, 13.9; HRMS (ESI) m/z: $[\mathrm{M}+\mathrm{Na}]^{+}$Calcd for $\mathrm{C}_{12} \mathrm{H}_{14} \mathrm{~N}_{6} \mathrm{Na}$ : 265.1172, Found 265.1174.

\section{1-(1-Azidopentan-2-yl)-2-(4-iodophenyl)diazene (4v)}<smiles>CC(C)C(CN)N=Nc1ccc(Cl)cc1</smiles>

The product was obtained after purification by column chromatography (gradient hexane/EtOAc $50: 1$ to $10: 1)$ as a yellow oil (42 mg, $83 \%$ yield); ${ }^{1} \mathrm{H}$ NMR $\left(500 \mathrm{MHz}, \mathrm{CDCl}_{3}\right) \delta 7.66(\mathrm{~d}, J=8.7 \mathrm{~Hz}$, $2 \mathrm{H}), 7.44(\mathrm{~d}, J=8.7 \mathrm{~Hz}, 2 \mathrm{H}), 3.89(\mathrm{dd}, J=4.9,3.5 \mathrm{~Hz}, 1 \mathrm{H}), 3.81(\mathrm{~d}, J=12.6 \mathrm{~Hz}, 1 \mathrm{H}), 3.61(\mathrm{~d}, J=$ $3.7 \mathrm{~Hz}, 1 \mathrm{H}), 1.95-1.87(\mathrm{~m}, 1 \mathrm{H}), 1.78-1.71(\mathrm{~m}, 1 \mathrm{H}), 1.32(\mathrm{q}, J=7.6 \mathrm{~Hz}, 2 \mathrm{H}), 0.93(\mathrm{t}, J=7.4 \mathrm{~Hz}$, 
$3 \mathrm{H}) ;{ }^{13} \mathrm{C} \mathrm{NMR}\left(126 \mathrm{MHz}, \mathrm{CDCl}_{3}\right) \delta 150.1,136.8,129.3,123.8,77.2,53.5,33.0,19.1,13.9$; HRMS (ESI) $\mathrm{m} / \mathrm{z}$ : $[\mathrm{M}+\mathrm{Na}]^{+}$Calcd for $\mathrm{C}_{11} \mathrm{H}_{14} \mathrm{ClN}_{5} \mathrm{Na}$ : 274.0830, Found 274.0840.

\section{1-(1-Azidopentan-2-yl)-2-(4-iodophenyl)diazene (4w)}<smiles>CC(C)C(CN)N=Nc1ccc(I)cc1</smiles>

The product was obtained after purification by column chromatography (gradient hexane/EtOAc $50: 1$ to $10: 1)$ as a yellow oil $\left(60 \mathrm{mg}, 87 \%\right.$ yield); ${ }^{1} \mathrm{H}$ NMR $\left(500 \mathrm{MHz}, \mathrm{CDCl}_{3}\right) \delta 7.81(\mathrm{~d}, J=8.5 \mathrm{~Hz}$, 2H), 7.44 (d, $J=8.6 \mathrm{~Hz}, 2 \mathrm{H}), 3.87$ (dd, $J=4.9,3.5 \mathrm{~Hz}, 1 \mathrm{H}), 3.79$ (dd, $J=12.6,8.1 \mathrm{~Hz}, 1 \mathrm{H}), 3.58$ $(\mathrm{dd}, J=12.6,3.7 \mathrm{~Hz}, 1 \mathrm{H}), 1.89(\mathrm{~d}, J=8.0 \mathrm{~Hz}, 1 \mathrm{H}), 1.78-1.70(\mathrm{~m}, 1 \mathrm{H}), 1.31(\mathrm{q}, J=7.6 \mathrm{~Hz}, 2 \mathrm{H})$, $0.92(\mathrm{t}, J=7.4 \mathrm{~Hz}, 3 \mathrm{H}) ;{ }^{13} \mathrm{C} \mathrm{NMR}\left(126 \mathrm{MHz}, \mathrm{CDCl}_{3}\right) \delta 151.1,138.3,124.1,97.5,77.0,53.5,32.9$, 19.1, 13.9; HRMS (ESI) m/z: [M+Na] ${ }^{+}$Calcd for $\mathrm{C}_{11} \mathrm{H}_{14} \mathrm{IN}_{5} \mathrm{Na}$ : 366.0186, Found 366.0195.

\section{1-(1-Azidopentan-2-yl)-2-(m-tolyl)diazene (4x)}<smiles>Cc1cccc(/N=N/C(CN)C(C)C)c1</smiles>

The product was obtained after purification by column chromatography (gradient hexane/EtOAc $50: 1$ to $10: 1)$ as a yellow oil (36 mg, $78 \%$ yield); ${ }^{1} \mathrm{H}$ NMR $\left(500 \mathrm{MHz}, \mathrm{CDCl}_{3}\right) \delta 7.51(\mathrm{~d}, J=8.4 \mathrm{~Hz}$, 2H), $7.35(\mathrm{t}, J=7.6 \mathrm{~Hz}, 1 \mathrm{H}), 7.28-7.24(\mathrm{~m}, 1 \mathrm{H}), 3.92-3.85(\mathrm{~m}, 1 \mathrm{H}), 3.84-3.77(\mathrm{~m}, 1 \mathrm{H}), 3.60$ $(\mathrm{dd}, J=12.5,3.8 \mathrm{~Hz}, 1 \mathrm{H}), 2.42(\mathrm{~s}, 3 \mathrm{H}), 1.93(\mathrm{dd}, J=13.9,7.4 \mathrm{~Hz}, 1 \mathrm{H}), 1.78-1.72(\mathrm{~m}, 1 \mathrm{H}), 1.33(\mathrm{q}$, $J=7.6 \mathrm{~Hz}, 2 \mathrm{H}), 0.93(\mathrm{t}, J=7.4 \mathrm{~Hz}, 3 \mathrm{H}) ;{ }^{13} \mathrm{C} \mathrm{NMR}\left(126 \mathrm{MHz}, \mathrm{CDCl}_{3}\right) \delta 139.0,131.5,128.8,122.6$, 119.9, 100.0, 77.2, 53.6, 33.0, 21.3, 19.1, 13.9; HRMS (ESI) m/z: $[\mathrm{M}+\mathrm{Na}]^{+}$Calcd for $\mathrm{C}_{12} \mathrm{H}_{17} \mathrm{~N}_{5} \mathrm{Na}$ : 254.1376, Found 254.1376.

\section{1-(1-Azidopentan-2-yl)-2-(3-chlorophenyl)diazene (4y)}<smiles>CCCC(CN)N=Nc1cccc(F)c1</smiles>

The product was obtained after purification by column chromatography (gradient hexane/EtOAc $50: 1$ to $10: 1)$ as a yellow oil $\left(39 \mathrm{mg}, 76 \%\right.$ yield); ${ }^{1} \mathrm{H}$ NMR $\left(500 \mathrm{MHz}, \mathrm{CDCl}_{3}\right) \delta 7.56(\mathrm{dt}, J=7.9$, $1.3 \mathrm{~Hz}, 1 \mathrm{H}), 7.45(\mathrm{td}, J=8.1,5.8 \mathrm{~Hz}, 1 \mathrm{H}), 7.38(\mathrm{dt}, J=9.6,2.2 \mathrm{~Hz}, 1 \mathrm{H}), 7.18-7.13(\mathrm{~m}, 1 \mathrm{H}), 3.91$ $(\mathrm{dd}, J=4.9,3.6 \mathrm{~Hz}, 1 \mathrm{H}), 3.81(\mathrm{dd}, J=12.6,8.1 \mathrm{~Hz}, 1 \mathrm{H}), 3.61(\mathrm{dd}, J=12.6,3.8 \mathrm{~Hz}, 1 \mathrm{H}), 1.95-$ $1.88(\mathrm{~m}, 1 \mathrm{H}), 1.80-1.72(\mathrm{~m}, 1 \mathrm{H}), 1.33(\mathrm{dt}, J=22.9,7.5 \mathrm{~Hz}, 1 \mathrm{H}), 0.93(\mathrm{t}, J=7.4 \mathrm{~Hz}, 3 \mathrm{H}) ;{ }^{13} \mathrm{C}$ $\operatorname{NMR}\left(126 \mathrm{MHz}, \mathrm{CDCl}_{3}\right) \delta 163.2\left(\mathrm{C}-\mathrm{F}, 1 \mathrm{~J}_{\mathrm{C}-\mathrm{F}}=247.9 \mathrm{~Hz}\right), 153.3\left(\mathrm{C}-\mathrm{F}, 3_{\mathrm{C}-\mathrm{F}}=12.3 \mathrm{~Hz}\right), 130.3(\mathrm{C}-\mathrm{F}$, $\left.3_{\mathrm{C}-\mathrm{F}}=8.3 \mathrm{~Hz}\right), 119.9\left(\mathrm{C}-\mathrm{F}, 4_{\mathrm{C}-\mathrm{F}}=3.0 \mathrm{~Hz}\right), 117.7\left(\mathrm{C}-\mathrm{F}, 2 \mathrm{~J}_{\mathrm{C}-\mathrm{F}}=22.1 \mathrm{~Hz}\right), 108.0\left(\mathrm{C}-\mathrm{F}, 2 \mathrm{~J}_{\mathrm{C}-\mathrm{F}}=23.1\right.$ $\mathrm{Hz}$ ), 76.9, 53.5, 33.0, 19.0, 13.9; ${ }^{19} \mathrm{~F}$ NMR (471 $\left.\mathrm{MHz}, \mathrm{CDCl}_{3}\right) \delta-111.94 ; \mathrm{HRMS}(\mathrm{ESI}) \mathrm{m} / \mathrm{z}$ : $[\mathrm{M}+\mathrm{Na}]^{+}$Calcd for $\mathrm{C}_{11} \mathrm{H}_{14} \mathrm{FN}_{5} \mathrm{Na}$ : 258.1125, Found 258.1126.

\section{1-(1-Azidopentan-2-yl)-2-(3-chlorophenyl)diazene (4z)}<smiles>CC(C)C(CN)N=Nc1cccc(Cl)c1</smiles>

The product was obtained after purification by column chromatography (gradient hexane/EtOAc 50:1 to $10: 1)$ as a yellow oil (38 mg, $76 \%$ yield); ${ }^{1} \mathrm{H} \mathrm{NMR}\left(500 \mathrm{MHz}, \mathrm{CDCl}_{3}\right) \delta 7.70-7.66(\mathrm{~m}, 1 \mathrm{H})$, 
$7.63(\mathrm{ddd}, J=5.6,3.0,1.8 \mathrm{~Hz}, 1 \mathrm{H}), 7.42(\mathrm{dd}, J=4.9,1.6 \mathrm{~Hz}, 2 \mathrm{H}), 3.94-3.88(\mathrm{~m}, 1 \mathrm{H}), 3.81(\mathrm{dd}, J$ $=12.6,8.1 \mathrm{~Hz}, 1 \mathrm{H}), 3.61(\mathrm{dd}, J=12.6,3.7 \mathrm{~Hz}, 1 \mathrm{H}), 1.97-1.87(\mathrm{~m}, 1 \mathrm{H}), 1.80-1.72(\mathrm{~m}, 1 \mathrm{H}), 1.34$ $-1.29(\mathrm{~m}, 2 \mathrm{H}), 0.93(\mathrm{t}, J=7.3 \mathrm{~Hz}, 3 \mathrm{H}) ;{ }^{13} \mathrm{C} \mathrm{NMR}\left(126 \mathrm{MHz}, \mathrm{CDCl}_{3}\right) \delta 152.6,135.1,130.7,130.1$, 121.9, 121.6, 76.9, 53.5, 32.9, 19.1, 13.9; HRMS (ESI) m/z: $[\mathrm{M}+\mathrm{Na}]^{+}$Calcd for $\mathrm{C}_{11} \mathrm{H}_{14} \mathrm{ClN}_{5} \mathrm{Na}$ : 274.0830 , Found 274.0821.

\section{1-(1-Azidopentan-2-yl)-2-(2-fluorophenyl)diazene (4aa)}<smiles>CCCC(CN)N=Nc1ccccc1F</smiles>

The product was obtained after purification by column chromatography (gradient hexane/EtOAc 50:1 to $10: 1)$ as a yellow oil (35 mg, $71 \%$ yield); ${ }^{1} \mathrm{H} \mathrm{NMR}\left(500 \mathrm{MHz}, \mathrm{CDCl}_{3}\right) \delta 7.51-7.39(\mathrm{~m}, 2 \mathrm{H})$, 7.23 (ddd, $J=10.6,8.3,1.2 \mathrm{~Hz}, 1 \mathrm{H}), 7.19-7.15(\mathrm{~m}, 1 \mathrm{H}), 3.99-3.92(\mathrm{~m}, 1 \mathrm{H}), 3.83$ (dd, $J=12.7$, $7.8 \mathrm{~Hz}, 1 \mathrm{H}), 3.62(\mathrm{dd}, J=12.7,4.0 \mathrm{~Hz}, 1 \mathrm{H}), 1.92(\mathrm{~d}, J=7.2 \mathrm{~Hz}, 1 \mathrm{H}), 1.79(\mathrm{~s}, 1 \mathrm{H}), 1.34(\mathrm{td}, J=7.5$, $6.0 \mathrm{~Hz}, 2 \mathrm{H}), 0.94(\mathrm{t}, J=7.3 \mathrm{~Hz}, 3 \mathrm{H}) ;{ }^{13} \mathrm{C} \mathrm{NMR}\left(126 \mathrm{MHz}, \mathrm{CDCl}_{3}\right) \delta 159.3\left(\mathrm{C}-\mathrm{F}, 1 \mathrm{~J}_{\mathrm{C}-\mathrm{F}}=256.6 \mathrm{~Hz}\right)$, $139.8\left(\mathrm{C}-\mathrm{F}, 3 \mathrm{~J}_{\mathrm{C}-\mathrm{F}}=7.3 \mathrm{~Hz}\right), 132.3\left(\mathrm{C}-\mathrm{F}, 3 \mathrm{~J}_{\mathrm{C}-\mathrm{F}}=8.2 \mathrm{~Hz}\right), 124.3\left(\mathrm{C}-\mathrm{F}, 4 \mathrm{~J}_{\mathrm{C}-\mathrm{F}}=4.0 \mathrm{~Hz}\right), 118.2,117.0$ $\left(\mathrm{C}-\mathrm{F}, 2 \mathrm{~J}_{\mathrm{C}-\mathrm{F}}=19.8 \mathrm{~Hz}\right), 77.4,53.6,33.0,19.0,13.9 ;{ }^{19} \mathrm{~F} \mathrm{NMR}\left(471 \mathrm{MHz}, \mathrm{CDCl}_{3}\right) \delta-125.60$; HRMS (ESI) m/z: [M+Na] $]^{+}$Calcd for $\mathrm{C}_{11} \mathrm{H}_{14} \mathrm{FN}_{5} \mathrm{Na}$ : 258.1125, Found 258.1131.

1-(1-Azidopentan-2-yl)-2-(2-iodophenyl)diazene (4ab)<smiles>CCCC(CN)N=Nc1ccccc1I</smiles>

The product was obtained after purification by column chromatography (gradient hexane/EtOAc $50: 1$ to $10: 1)$ as a yellow oil (45 $\mathrm{mg}, 66 \%$ yield); ${ }^{1} \mathrm{H} \mathrm{NMR}\left(500 \mathrm{MHz}, \mathrm{CDCl}_{3}\right) \delta 7.98(\mathrm{dd}, J=7.9$, $1.2 \mathrm{~Hz}, 1 \mathrm{H}), 7.37(\mathrm{~d}, J=7.9 \mathrm{~Hz}, 1 \mathrm{H}), 7.27(\mathrm{~d}, J=1.5 \mathrm{~Hz}, 1 \mathrm{H}), 7.14$ (d, $J=1.5 \mathrm{~Hz}, 1 \mathrm{H}), 4.05$ (s, $1 \mathrm{H}), 3.87-3.81(\mathrm{~m}, 1 \mathrm{H}), 3.64(\mathrm{dd}, J=12.7,4.1 \mathrm{~Hz}, 1 \mathrm{H}), 1.99-1.93(\mathrm{~m}, 1 \mathrm{H}), 1.77(\mathrm{~d}, J=7.0 \mathrm{~Hz}$, $1 \mathrm{H}), 1.40-1.33(\mathrm{~m}, 2 \mathrm{H}), 0.97-0.94(\mathrm{~m}, 3 \mathrm{H}) ;{ }^{13} \mathrm{C} \mathrm{NMR}\left(126 \mathrm{MHz}, \mathrm{CDCl}_{3}\right) \delta 151.4,139.6,131.8$, 129.0, 117.8, 99.5, 76.5, 53.7, 32.9, 19.1, 13.9; HRMS (ESI) m/z: $[\mathrm{M}+\mathrm{Na}]^{+}$Calcd for $\mathrm{C}_{11} \mathrm{H}_{14} \mathrm{IN}_{5} \mathrm{Na}$ : 366.0186 , Found 366.0195.

1-(1-Azidopentan-2-yl)-2-(2-nitrophenyl)diazene (4ac)<smiles>CCCC(CN)N=Nc1ccccc1[N+](=O)[O-]</smiles>

The product was obtained after purification by column chromatography (gradient hexane/EtOAc $50: 1$ to $10: 1)$ as a yellow oil (39 $\mathrm{mg}, 75 \%$ yield); ${ }^{1} \mathrm{H}$ NMR (500 $\left.\mathrm{MHz}, \mathrm{CDCl}_{3}\right) \delta 7.88(\mathrm{dd}, J=8.1$, $1.3 \mathrm{~Hz}, 1 \mathrm{H}), 7.59(\mathrm{td}, J=7.7,1.3 \mathrm{~Hz}, 1 \mathrm{H}), 7.48(\mathrm{td}, J=7.8,1.4 \mathrm{~Hz}, 1 \mathrm{H}), 7.23(\mathrm{dd}, J=7.9,1.4 \mathrm{~Hz}$, $1 \mathrm{H}), 3.97-3.90(\mathrm{~m}, 1 \mathrm{H}), 3.78(\mathrm{dd}, J=12.7,8.0 \mathrm{~Hz}, 1 \mathrm{H}), 3.59(\mathrm{dd}, J=12.7,3.9 \mathrm{~Hz}, 1 \mathrm{H}), 1.96-$ $1.87(\mathrm{~m}, 1 \mathrm{H}), 1.76-1.66(\mathrm{~m}, 1 \mathrm{H}), 1.36-1.27(\mathrm{~m}, 2 \mathrm{H}), 0.89(\mathrm{t}, J=7.3 \mathrm{~Hz}, 3 \mathrm{H}) ;{ }^{13} \mathrm{C}$ NMR $(126$ $\left.\mathrm{MHz}, \mathrm{CDCl}_{3}\right) \delta 145.7,133.6,130.1,124.2,119.3,77.5,53.4,32.8,19.0,13.9 ; \mathrm{HRMS}(\mathrm{ESI}) \mathrm{m} / \mathrm{z}$ : $[\mathrm{M}+\mathrm{Na}]^{+}$Calcd for $\mathrm{C}_{11} \mathrm{H}_{14} \mathrm{~N}_{6} \mathrm{O}_{2} \mathrm{Na}$ : 285.1070, Found 285.1045.

1-(1-Azidopentan-2-yl)-2-(naphthalen-1-yl)diazene (4ad) 
<smiles>CC(C)C(CN)N=Nc1cccc2ccccc12</smiles>

The product was obtained after purification by column chromatography (gradient hexane/EtOAc $50: 1$ to $10: 1)$ as a yellow oil (38 mg, $71 \%$ yield); ${ }^{1} \mathrm{H}$ NMR $\left(500 \mathrm{MHz}, \mathrm{CDCl}_{3}\right) \delta 8.72(\mathrm{~d}, J=8.9 \mathrm{~Hz}$, 1H), $7.95(\mathrm{~d}, J=7.7 \mathrm{~Hz}, 1 \mathrm{H}), 7.91(\mathrm{~d}, J=8.1 \mathrm{~Hz}, 1 \mathrm{H}), 7.63(\mathrm{t}, J=6.9 \mathrm{~Hz}, 1 \mathrm{H}), 7.59-7.56(\mathrm{~m}, 1 \mathrm{H})$, $7.53-7.46(\mathrm{~m}, 2 \mathrm{H}), 4.12-4.06(\mathrm{~m}, 1 \mathrm{H}), 3.91(\mathrm{dd}, J=12.7,8.2 \mathrm{~Hz}, 1 \mathrm{H}), 3.69(\mathrm{dd}, J=12.7,4.0 \mathrm{~Hz}$, $1 \mathrm{H}), 2.02(\mathrm{~d}, J=6.4 \mathrm{~Hz}, 1 \mathrm{H}), 1.81(\mathrm{~d}, J=7.7 \mathrm{~Hz}, 1 \mathrm{H}), 1.44-1.36(\mathrm{~m}, 2 \mathrm{H}), 0.97(\mathrm{t}, J=7.4 \mathrm{~Hz}, 3 \mathrm{H})$; ${ }^{13} \mathrm{C}$ NMR $\left(126 \mathrm{MHz}, \mathrm{CDCl}_{3}\right) \delta 147.4,134.1,130.9,130.3,127.9,126.9,126.5,125.5,123.2,112.3$, 77.2, 53.8, 33.1, 19.1, 13.9; HRMS (ESI) m/z: [M+Na $]^{+}$Calcd for $\mathrm{C}_{15} \mathrm{H}_{17} \mathrm{~N}_{5} \mathrm{Na}$ : 290.1376, Found 290.1374 .

((6-Azido-5-((4-methoxyphenyl)diazenyl)hexyl)oxy)-2H-chromen-2-one (4ae)<smiles>COc1ccc(/N=N/C(CN)CCCCOc2cc(=O)oc3ccccc23)cc1</smiles>

The product was obtained after purification by column chromatography (gradient hexane/EtOAc $20: 1$ to $3: 1)$ as a yellow oil (69 $\mathrm{mg}, 84 \%$ yield); ${ }^{1} \mathrm{H} \mathrm{NMR}\left(500 \mathrm{MHz}, \mathrm{CDCl}_{3}\right) \delta 7.69(\mathrm{~d}, J=8.9 \mathrm{~Hz}$, 3H), 7.52 (d, $J=1.4 \mathrm{~Hz}, 1 \mathrm{H}), 7.29(\mathrm{~d}, J=8.8 \mathrm{~Hz}, 1 \mathrm{H}), 7.19(\mathrm{~s}, 1 \mathrm{H}), 6.97-6.91(\mathrm{~m}, 2 \mathrm{H}), 5.62(\mathrm{~s}$, 1H), 4.09 (s, 2H), $3.85(\mathrm{~s}, 4 \mathrm{H}), 3.80(\mathrm{~s}, 1 \mathrm{H}), 3.61(\mathrm{~d}, J=4.2 \mathrm{~Hz}, 1 \mathrm{H}), 2.06(\mathrm{~d}, J=7.4 \mathrm{~Hz}, 1 \mathrm{H}), 1.97$ $-1.93(\mathrm{~m}, 1 \mathrm{H}), 1.88(\mathrm{~d}, J=7.1 \mathrm{~Hz}, 2 \mathrm{H}), 1.56-1.49(\mathrm{~m}, 2 \mathrm{H}) ;{ }^{13} \mathrm{C} \mathrm{NMR}\left(126 \mathrm{MHz}, \mathrm{CDCl}_{3}\right) \delta 165.6$, 163.0, 162.0, 153.3, 145.9, 132.3, 124.3, 123.8, 122.9, 116.7, 115.7, 114.1, 90.4, 76.1, 68.9, 55.6, 53.7, 30.5, 28.3, 22.3; HRMS (ESI) $\mathrm{m} / \mathrm{z}$ : $[\mathrm{M}+\mathrm{Na}]^{+}$Calcd for $\mathrm{C}_{22} \mathrm{H}_{23} \mathrm{~N}_{5} \mathrm{O}_{4} \mathrm{Na}$ : 444.1642, Found 444.1642 .

1-(6-Bromo-2-((4-methoxyphenyl)diazenyl)hexyl)-4-phenyl-1H-1,2,3-triazole (4af)<smiles>COc1ccc(N=NC(CCCCBr)Cn2cc(-c3ccccc3)nn2)cc1</smiles>

The product was obtained after purification by column chromatography (gradient hexane/EtOAc 20:1 to 5:1) as a yellow oil (56 mg, 64\% yield); $\left.{ }^{1} \mathrm{H} \mathrm{NMR} \mathrm{(500} \mathrm{MHz,} \mathrm{CDCl}_{3}\right) \delta 7.67(\mathrm{~d}, J=7.6 \mathrm{~Hz}$, 2H), 7.59 (d, $J=7.5 \mathrm{~Hz}, 3 \mathrm{H}), 7.31(\mathrm{t}, J=7.6 \mathrm{~Hz}, 2 \mathrm{H}), 7.23(\mathrm{~d}, J=7.4 \mathrm{~Hz}, 1 \mathrm{H}), 6.87(\mathrm{~d}, J=8.5 \mathrm{~Hz}$, 2H), $4.86(\mathrm{dd}, J=14.1,7.7 \mathrm{~Hz}, 1 \mathrm{H}), 4.72(\mathrm{dd}, J=14.1,4.2 \mathrm{~Hz}, 1 \mathrm{H}), 4.04(\mathrm{~d}, J=7.1 \mathrm{~Hz}, 2 \mathrm{H}), 3.78$ $(\mathrm{s}, 3 \mathrm{H}), 3.30(\mathrm{t}, J=6.7 \mathrm{~Hz}, 1 \mathrm{H}), 1.88-1.73(\mathrm{~m}, 4 \mathrm{H}), 1.45(\mathrm{~d}, J=9.7 \mathrm{~Hz}, 2 \mathrm{H}) ;{ }^{13} \mathrm{C} \mathrm{NMR}(126 \mathrm{MHz}$, $\left.\mathrm{CDCl}_{3}\right) \delta 162.1,147.7,145.8,130.6,128.8,128.1,125.7,124.3,120.5,114.2,75.7,55.6,52.7,33.2$, 32.3, 30.1, 24.3, 21.1; HRMS (ESI) m/z: $[\mathrm{M}+\mathrm{Na}]^{+}$Calcd for $\mathrm{C}_{21} \mathrm{H}_{24} \mathrm{BrN}_{5} \mathrm{ONa}$ : 464.1056, Found 464.1051.

\section{Cyclohexane-1,2-diamine (4ag)}


$\overbrace{}^{\mathrm{NH}_{2}}$

The product was obtained after purification by column chromatography (gradient hexane/EtOAc 100:1) as a yellow oil (69 mg, 84\% yield); ${ }^{1} \mathrm{H}$ NMR (500 MHz, $\left.\mathrm{CDCl}_{3}\right) \delta 2.88-2.82(\mathrm{~m}, 1 \mathrm{H}), 2.24$ $(\mathrm{d}, J=9.3 \mathrm{~Hz}, 2 \mathrm{H}), 1.84(\mathrm{dt}, J=13.0,2.6 \mathrm{~Hz}, 2 \mathrm{H}), 1.74-1.64(\mathrm{~m}, 2 \mathrm{H}), 1.58-1.49(\mathrm{~m}, 3 \mathrm{H}), 1.30-$ $1.24(\mathrm{~m}, 2 \mathrm{H}), 1.13(\mathrm{~d}, J=7.7 \mathrm{~Hz}, 2 \mathrm{H})$;

6-Azido-7-((4-methoxyphenyl)diazenyl)-3,7-dimethyloctan-1-ol (4ah)<smiles>COc1ccc(N=NC(C)(C)C(N)CC[C@H](C)CCO)cc1</smiles>

The product was obtained after purification by column chromatography (gradient hexane/EtOAc $20: 1$ to $6: 1)$ as a yellow oil $1: 1 \mathrm{dr}(56 \mathrm{mg}, 84 \%$ yield $) ;{ }^{1} \mathrm{H}$ NMR $\left(500 \mathrm{MHz}, \mathrm{CDCl}_{3}\right) \delta 7.73-7.65(\mathrm{~m}$, $2 \mathrm{H}), 6.99-6.92(\mathrm{~m}, 2 \mathrm{H}), 3.84(\mathrm{~s}, 3 \mathrm{H}), 3.72-3.65(\mathrm{~m}, 3 \mathrm{H}), 1.66-1.56(\mathrm{~m}, 5 \mathrm{H}), 1.39(\mathrm{~s}, 2 \mathrm{H}), 1.27$ $(\mathrm{s}, 3 \mathrm{H}), 1.24(\mathrm{~s}, 3 \mathrm{H}), 0.92(\mathrm{t}, J=6.0 \mathrm{~Hz}, 3 \mathrm{H}) ;{ }^{13} \mathrm{C} \mathrm{NMR}\left(126 \mathrm{MHz}, \mathrm{CDCl}_{3}\right)$ Mixture of diastereomers is observed. Ratio: 5/5. Major diastereomer: $\delta 161.6,146.2,124.1,114.0,73.5,71.3$, 60.9, 55.5, 40.0, 34.9, 29.6, 27.1, 22.1, 21.6, 19.8; Minor diastereomer: $\delta$ 161.6, 146.2, 124.1, 114.0, 73.4 , 70.9, 60.9, 55.5, 39.4, 34.5, 29.2, 26.9, 22.1, 21.6, 19.4; HRMS (ESI) m/z: $[\mathrm{M}+\mathrm{Na}]^{+}$Calcd for $\mathrm{C}_{17} \mathrm{H}_{27} \mathrm{~N}_{5} \mathrm{O}_{2} \mathrm{Na}: 356.2057$, Found 356.2060.

1-Azido-2-((4-methoxyphenyl)diazenyl)propan-2-yl)-4,4a-dimethyl-4,4a,5,6,7,8-hexahydronap hthalen-2(3H)-one (4ai)<smiles>COc1ccc(/N=N/C(C)(CN)C2CCC3=CC(=O)C[C@H](C)[C@]3(C)C2)cc1</smiles>

The product was obtained after purification by column chromatography (gradient hexane/EtOAc $20: 1$ to $6: 1)$ as a yellow oil $1: 1 \mathrm{dr}(64 \mathrm{mg}, 81 \%$ yield $) ;{ }^{1} \mathrm{H}$ NMR $\left(500 \mathrm{MHz}, \mathrm{CDCl}_{3}\right) \delta 7.64(\mathrm{~d}, J=$ $8.6 \mathrm{~Hz}, 2 \mathrm{H}), 6.90(\mathrm{~d}, J=8.9 \mathrm{~Hz}, 2 \mathrm{H}), 5.68-5.66(\mathrm{~m}, 0.8 \mathrm{H}), 5.22(\mathrm{~d}, J=2.0 \mathrm{~Hz}, 0.3 \mathrm{H}), 3.79(\mathrm{~s}, 3 \mathrm{H})$, $3.54(\mathrm{~d}, J=13.0 \mathrm{~Hz}, 1 \mathrm{H}), 3.49(\mathrm{~s}, 1 \mathrm{H}), 2.46-2.11(\mathrm{~m}, 6 \mathrm{H}), 1.98-1.84(\mathrm{~m}, 3 \mathrm{H}), 1.21-1.17(\mathrm{~m}$, $1 \mathrm{H}), 1.14(\mathrm{~d}, J=11.7 \mathrm{~Hz}, 3 \mathrm{H}), 1.04(\mathrm{~d}, J=23.6 \mathrm{~Hz}, 3 \mathrm{H}), 0.90(\mathrm{~d}, J=4.7 \mathrm{~Hz}, 2 \mathrm{H}), 0.84(\mathrm{~d}, J=6.8$ $\mathrm{Hz}, 1 \mathrm{H}) ;{ }^{13} \mathrm{C} \mathrm{NMR}\left(126 \mathrm{MHz}, \mathrm{CDCl}_{3}\right)$ Mixture of diastereomers is observed. Ratio: 5/5. Major diastereomer: $\delta 199.5,170.2,161.8,145.9,124.6,124.2,114.1,74.3,56.7,55.6,42.0,40.5,39.4$, 39.3, 38.3, 32.9, 27.0, 17.3, 16.8, 15.0; Minor diastereomer: $\delta 199.5,170.2,161.8,145.9,124.6$, 124.1, 114.1, 74.1, 56.6, 46.0, 42.1, 40.5, 39.3, 39.0, 38.5, 32.9, 27.5, 17.7, 16.8, 15.0; HRMS (ESI) $\mathrm{m} / \mathrm{z}:[\mathrm{M}+\mathrm{Na}]^{+}$Calcd for $\mathrm{C}_{22} \mathrm{H}_{29} \mathrm{~N}_{5} \mathrm{O}_{2} \mathrm{Na}$ : 418.2213, Found 418.2214.

1-(6-Bromo-2-((4-methoxyphenyl)diazenyl)hexyl)-4-phenyl-1H-1,2,3-triazole (4aj)<smiles>CCOC(=O)C1(C(=O)OCC)CC(CN)C(CN=Nc2ccc(OC)cc2)C1</smiles> 
The product was obtained after purification by column chromatography (gradient hexane/EtOAc $20: 1$ to $5: 1)$ as a yellow oil (51 mg, $62 \%$ yield); ${ }^{1} \mathrm{H}$ NMR $\left(500 \mathrm{MHz}, \mathrm{DMSO}-d_{6}\right) \delta 7.64(\mathrm{~d}, J=8.9$ $\mathrm{Hz}, 2 \mathrm{H}), 7.06(\mathrm{~d}, J=8.8 \mathrm{~Hz}, 2 \mathrm{H}), 4.12(\mathrm{dq}, J=10.5,7.0 \mathrm{~Hz}, 5 \mathrm{H}), 3.89-3.82(\mathrm{~m}, 4 \mathrm{H}), 3.56(\mathrm{dd}, J=$ 12.3, $6.3 \mathrm{~Hz}, 1 \mathrm{H}), 3.41(\mathrm{dd}, J=12.4,8.0 \mathrm{~Hz}, 1 \mathrm{H}), 2.70(\mathrm{~d}, J=7.3 \mathrm{~Hz}, 1 \mathrm{H}), 2.45-2.33(\mathrm{~m}, 3 \mathrm{H})$, $2.19-2.09(\mathrm{~m}, 2 \mathrm{H}), 1.16(\mathrm{t}, J=6.8 \mathrm{~Hz}, 6 \mathrm{H}) ;{ }^{13} \mathrm{C}$ NMR $\left(126 \mathrm{MHz}, \mathrm{DMSO}-d_{6}\right) \delta 172.1,171.8,161.8$, 146.0, 124.2, 114.8, 68.7, 61.7, 61.7, 58.7, 56.0, 51.6, 41.0, 37.5, 37.2, 14.3; HRMS (ESI) m/z: $[\mathrm{M}+\mathrm{Na}]^{+}$Calcd for $\mathrm{C}_{20} \mathrm{H}_{27} \mathrm{~N}_{5} \mathrm{O}_{5} \mathrm{Na}: 440.1904$, Found 440.1901 . 


\section{Copies of ${ }^{1} \mathrm{H},{ }^{13} \mathrm{C}$ and ${ }^{19} \mathrm{~F}$ NMR Spectra}

\section{4a ${ }^{1}$ H NMR (500 MHz, Chloroform-d)}

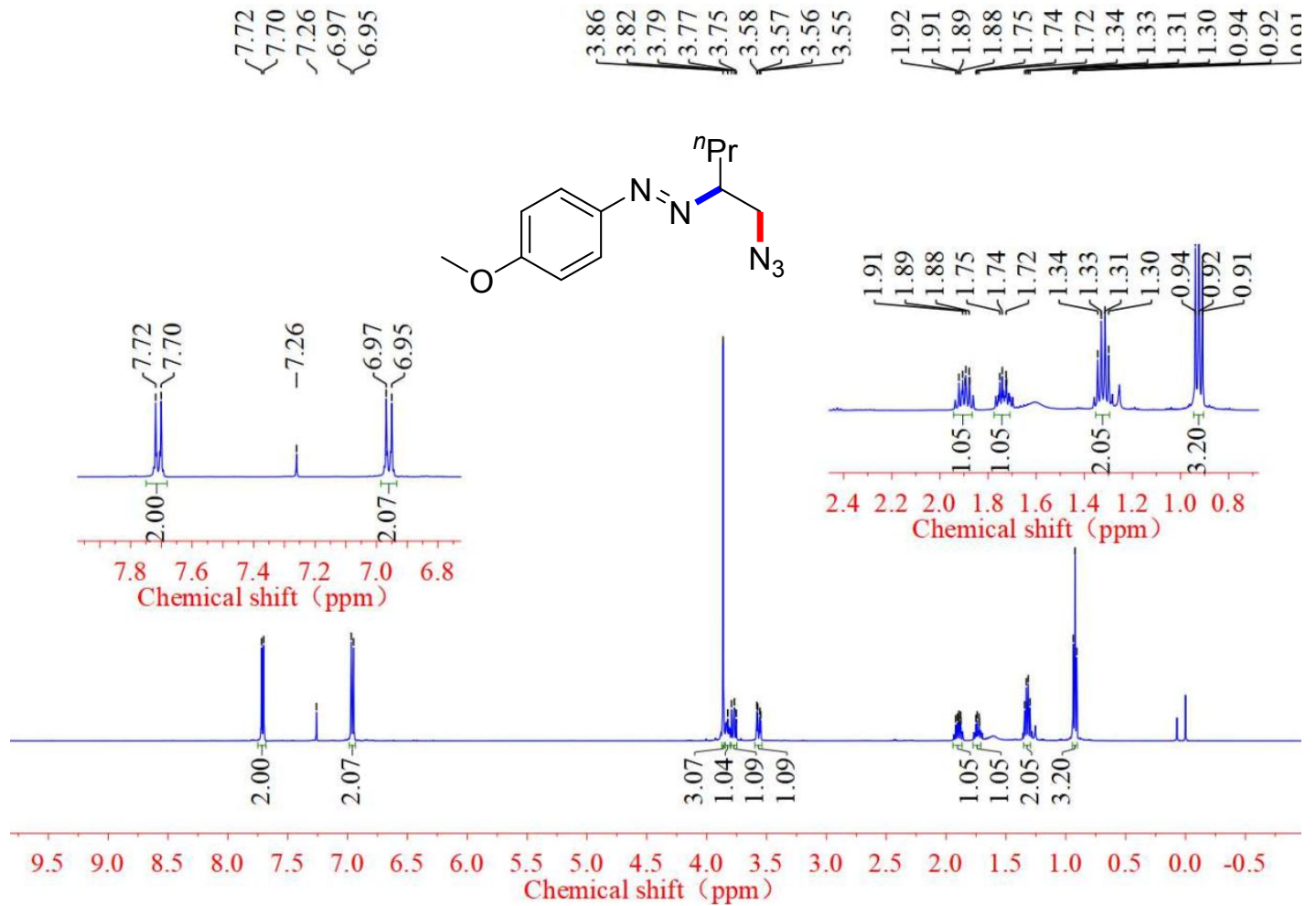

\section{4a ${ }^{13}$ C NMR (126 MHz, Chloroform-d)}

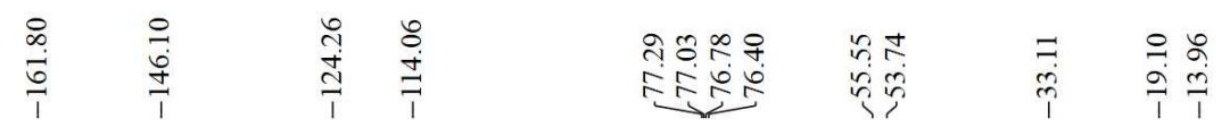

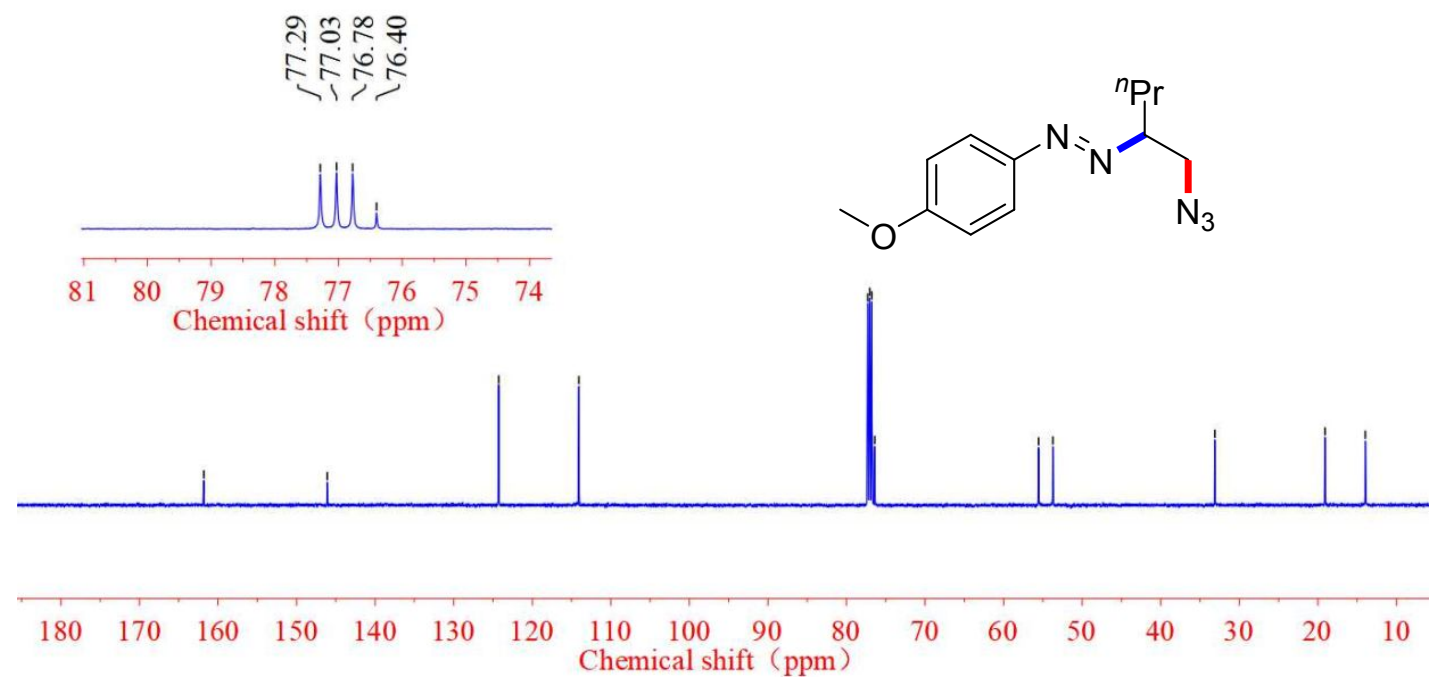




\section{4b ${ }^{1} \mathrm{H}$ NMR (500 MHz, Chloroform-d)}

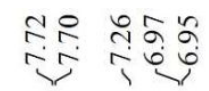

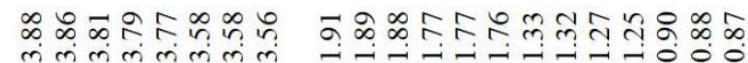

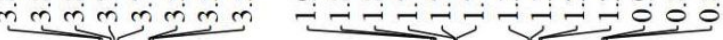

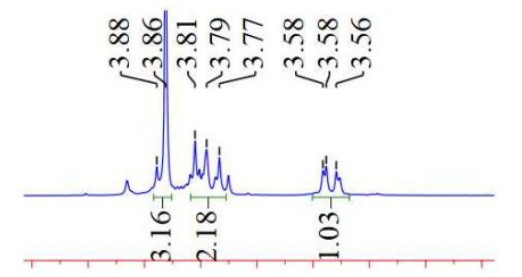

$\begin{array}{llllllllll}4.1 & 4.0 & 3.9 & 3.8 & 3.7 & 3.6 & 3.5 & 3.4 & 3.3\end{array}$

Chemical shift (ppm)<smiles>CCC(CN)N=Nc1ccc(OC)cc1</smiles>

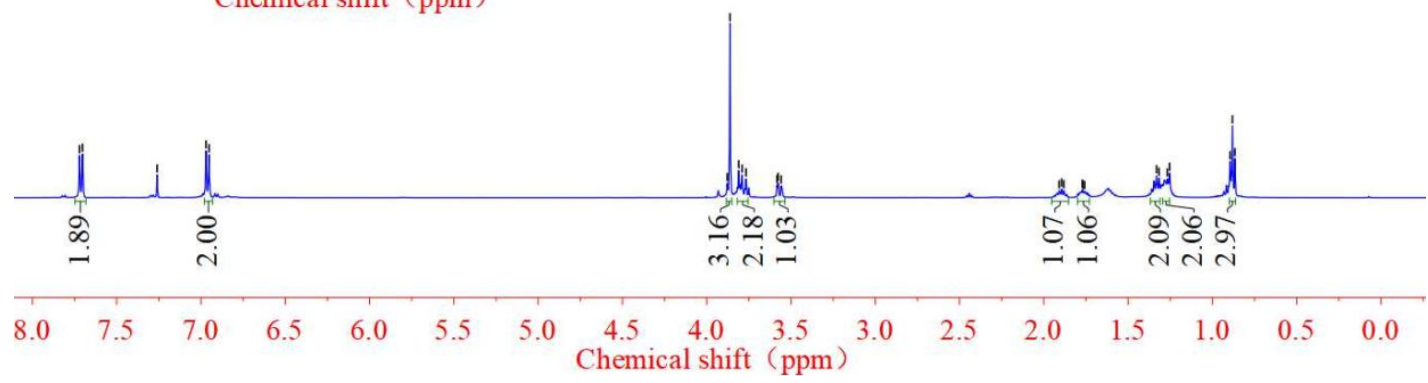

\section{4b ${ }^{13}$ C NMR (126 MHz, Chloroform-d)}

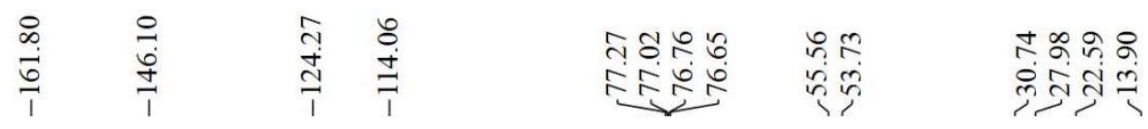
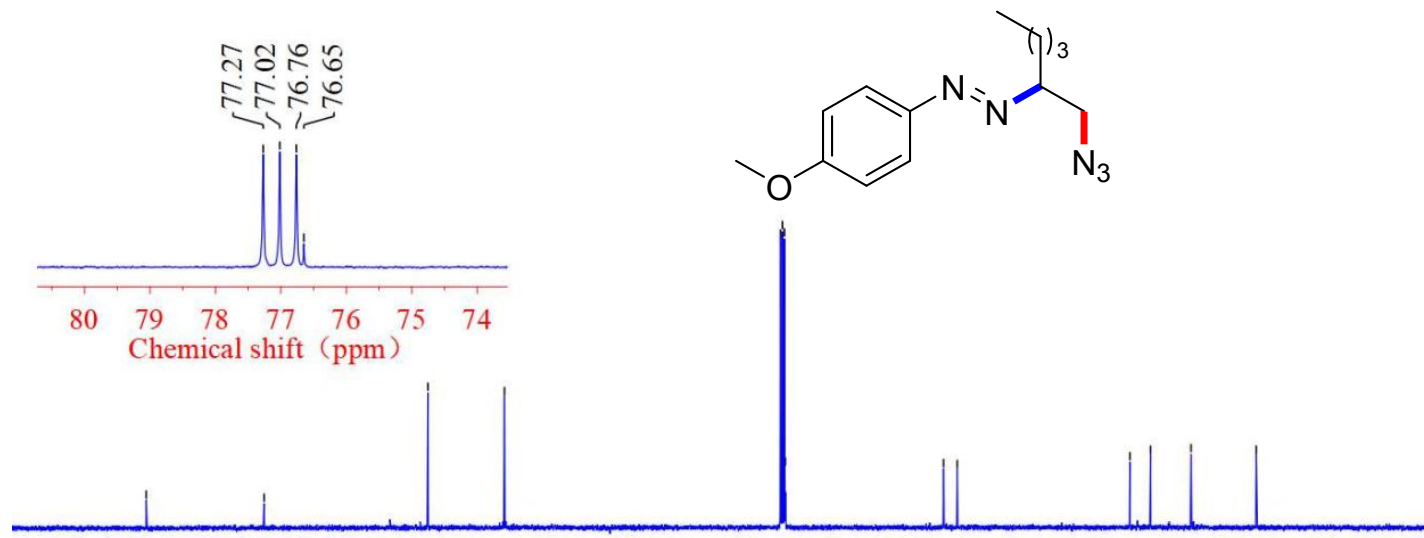

$\begin{array}{llllllllllllllll}170 & 160 & 150 & 140 & 130 & 120 & 110 & \begin{array}{c}100 \\ \text { Chemical shift }(\mathrm{ppm})\end{array}\end{array}$ 


\section{4c ${ }^{1}$ H NMR (500 MHz, Chloroform-d)}

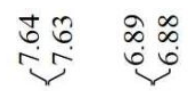

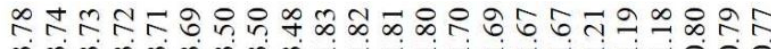

लंखिलालmलm -<smiles>CCC(CN)N=Nc1ccc(OC)cc1</smiles>

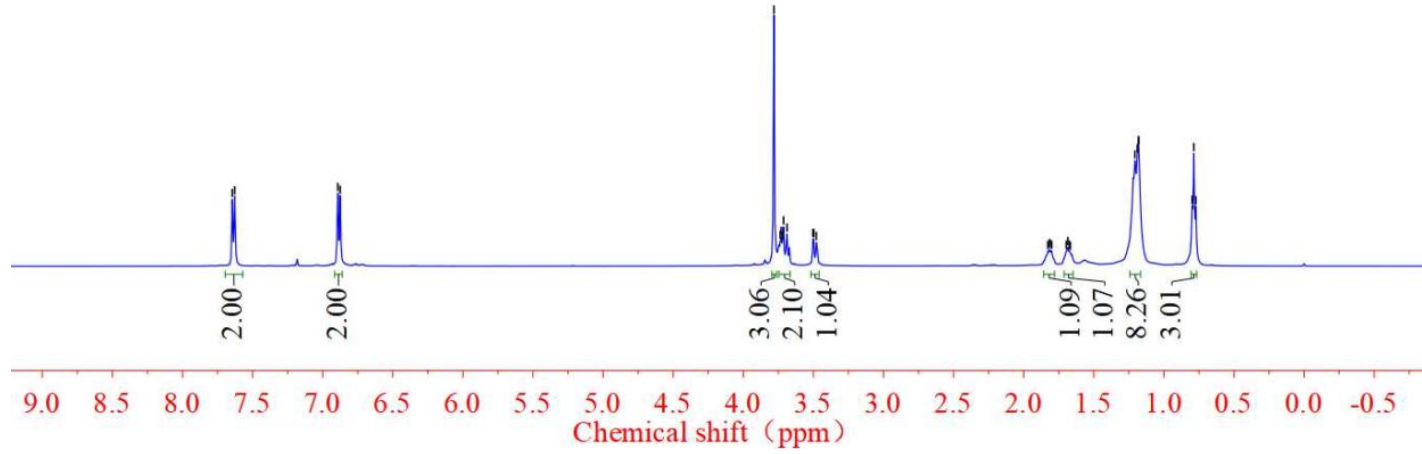

4c ${ }^{13}$ C NMR (126 MHz, Chloroform-d)

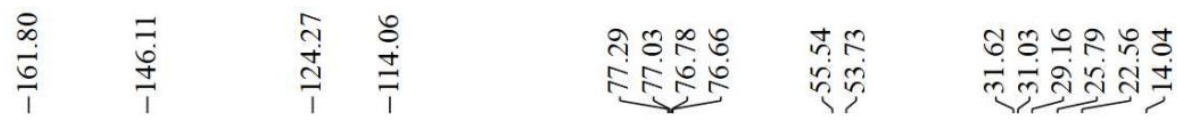
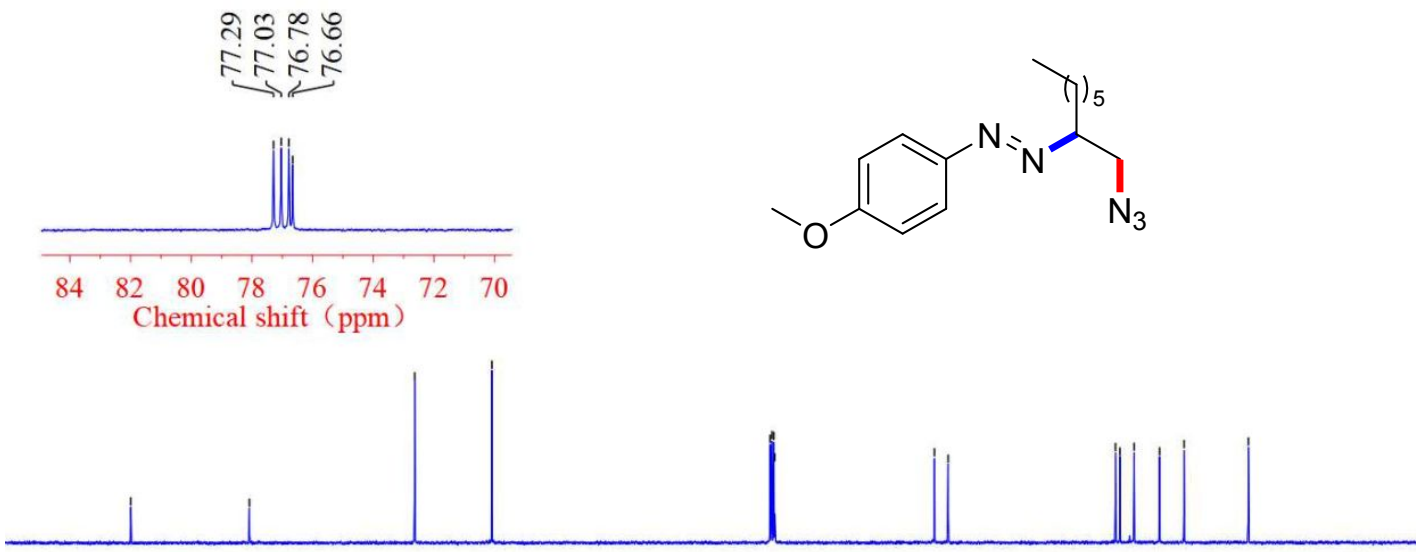

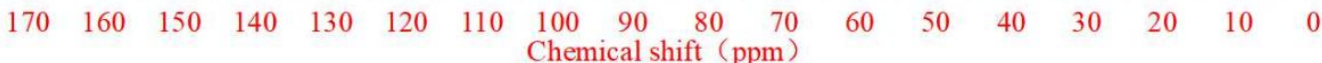




\section{4d ${ }^{1}$ H NMR (500 MHz, Chloroform-d)}

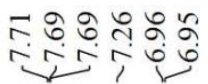

ลุํำळ

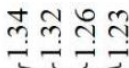
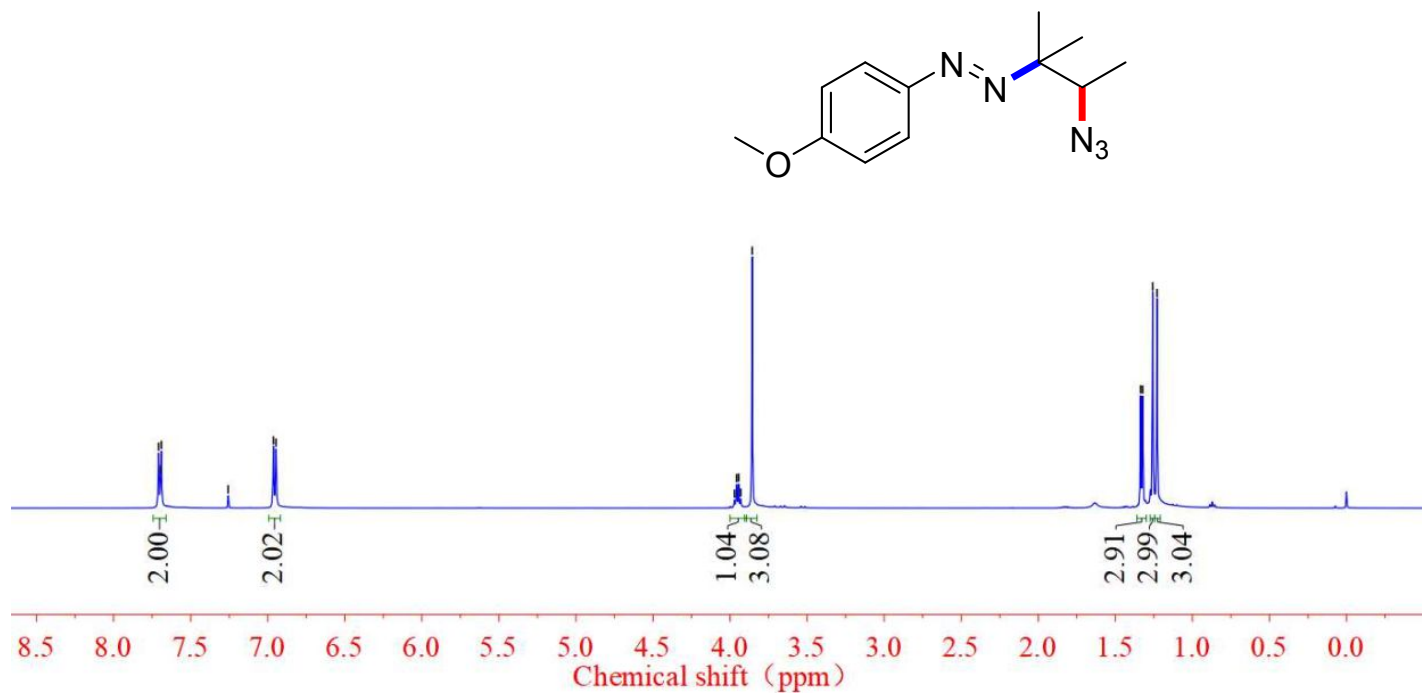

4d ${ }^{13}$ C NMR (126 MHz, Chloroform-d)

\begin{tabular}{|c|c|c|c|}
\hline $\begin{array}{l}\stackrel{+}{\sim} \\
\underset{0}{ \pm} \\
\stackrel{1}{+}\end{array}$ & 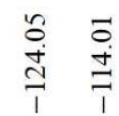 & 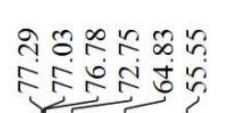 & 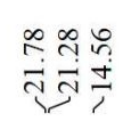 \\
\hline
\end{tabular}

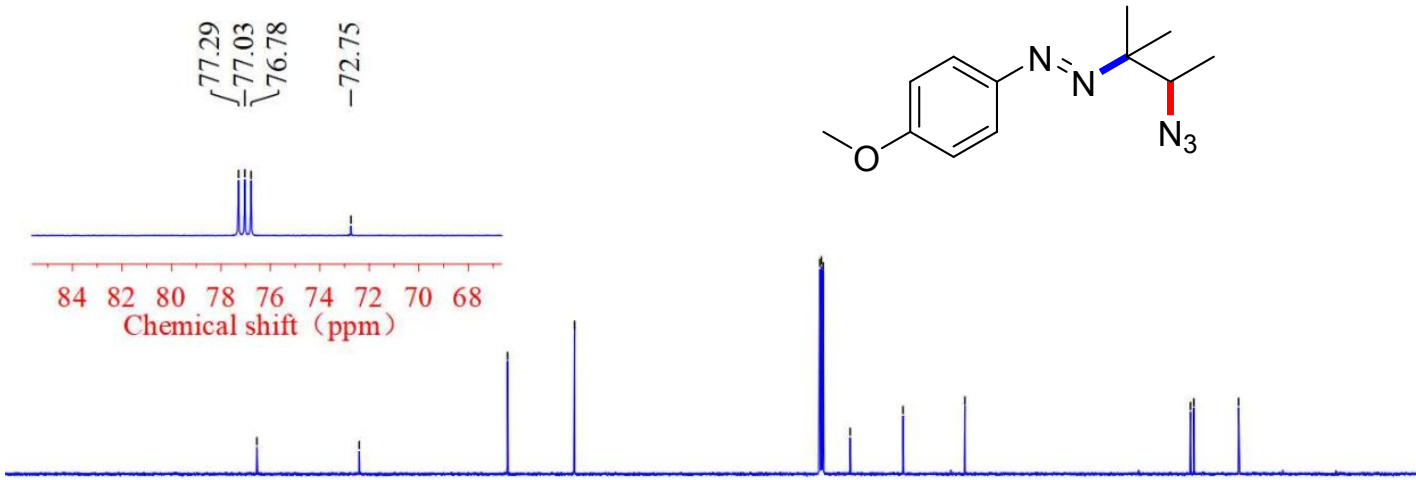

$\begin{array}{lllllllllllllllllllll}190 & 180 & 170 & 160 & 150 & 140 & 130 & 120 & 110 & 100 & 90 & 80 & 70 & 60 & 50 & 40 & 30 & 20 & 10 & 0 & -1(\end{array}$ Chemical shift (ppm) 


\section{4e ${ }^{1} \mathrm{H}$ NMR (500 MHz, Chloroform-d)}

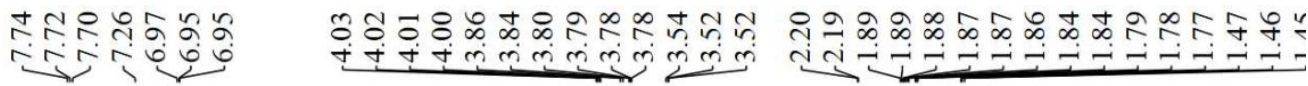

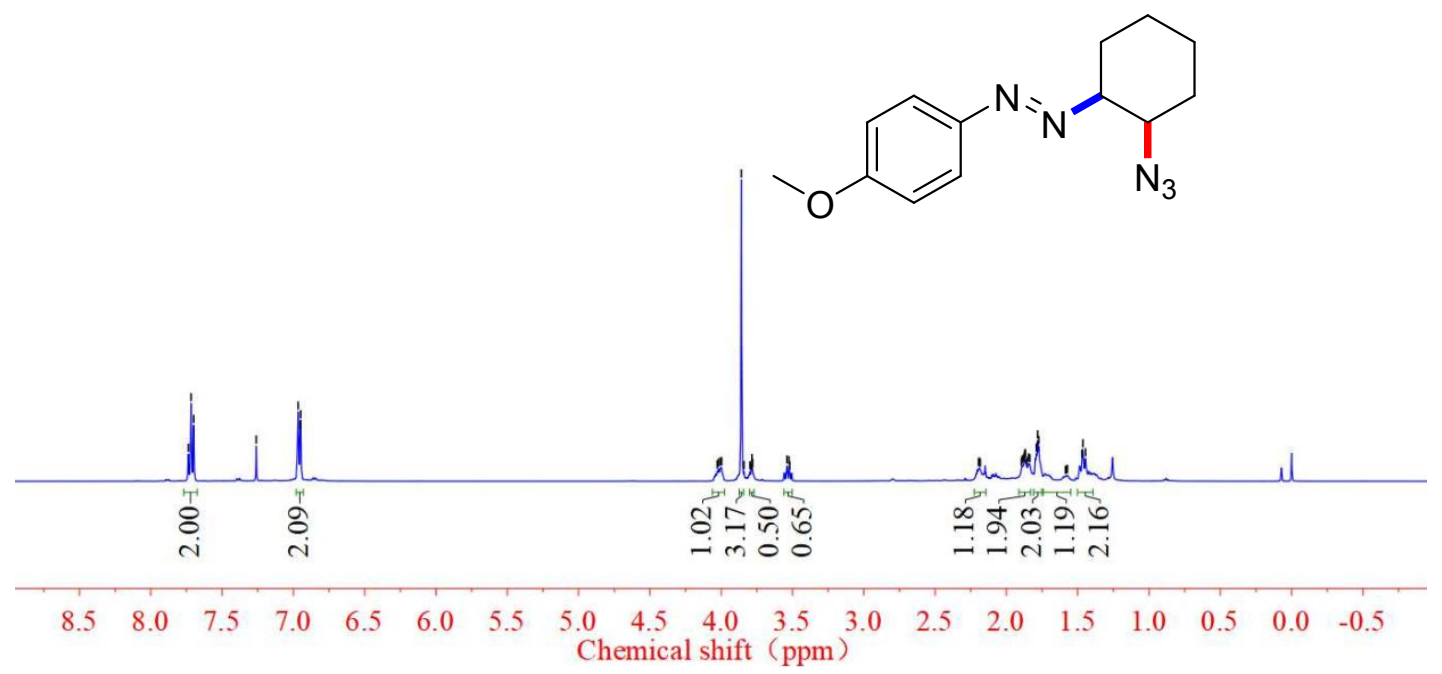

4e ${ }^{13}$ C NMR (126 MHz, Chloroform-d)

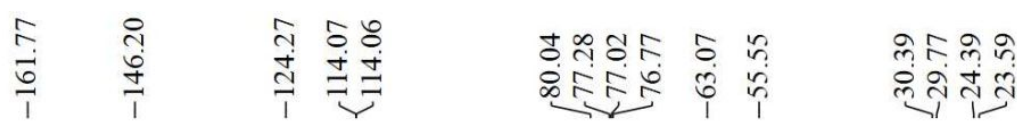

¿.

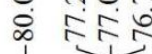<smiles>COc1ccc(N=NC2CCCCC2N)cc1</smiles>
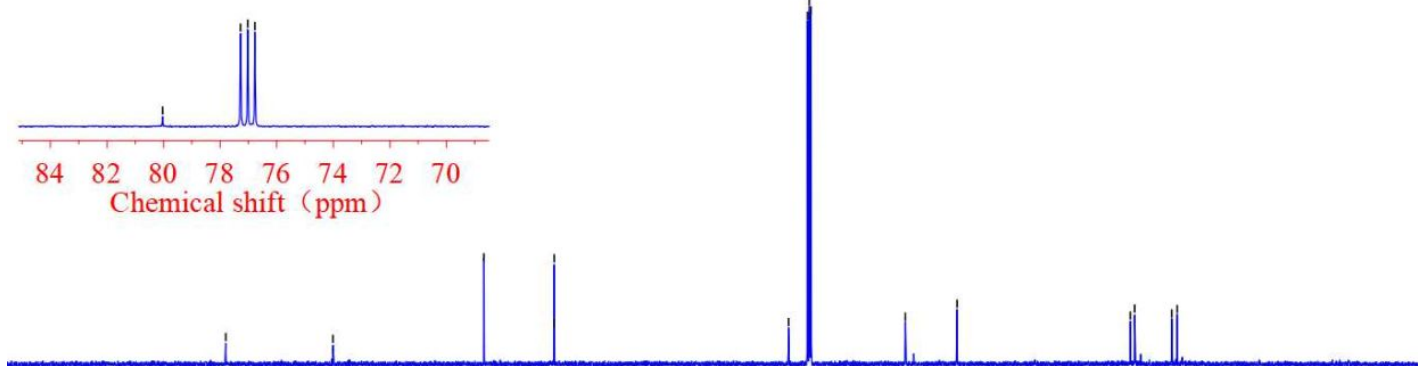

$\left.\begin{array}{llllllllllllllllllll}190 & 180 & 170 & 160 & 150 & 140 & 130 & 120 & 110 & 100 & 90 & 80 \\ \text { Chemical shift } & (\mathrm{ppm})\end{array}\right]$ 


\section{4f ${ }^{1} \mathrm{H}$ NMR (500 MHz, Chloroform-d)}

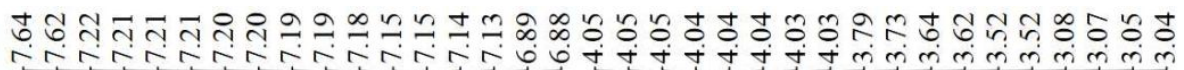

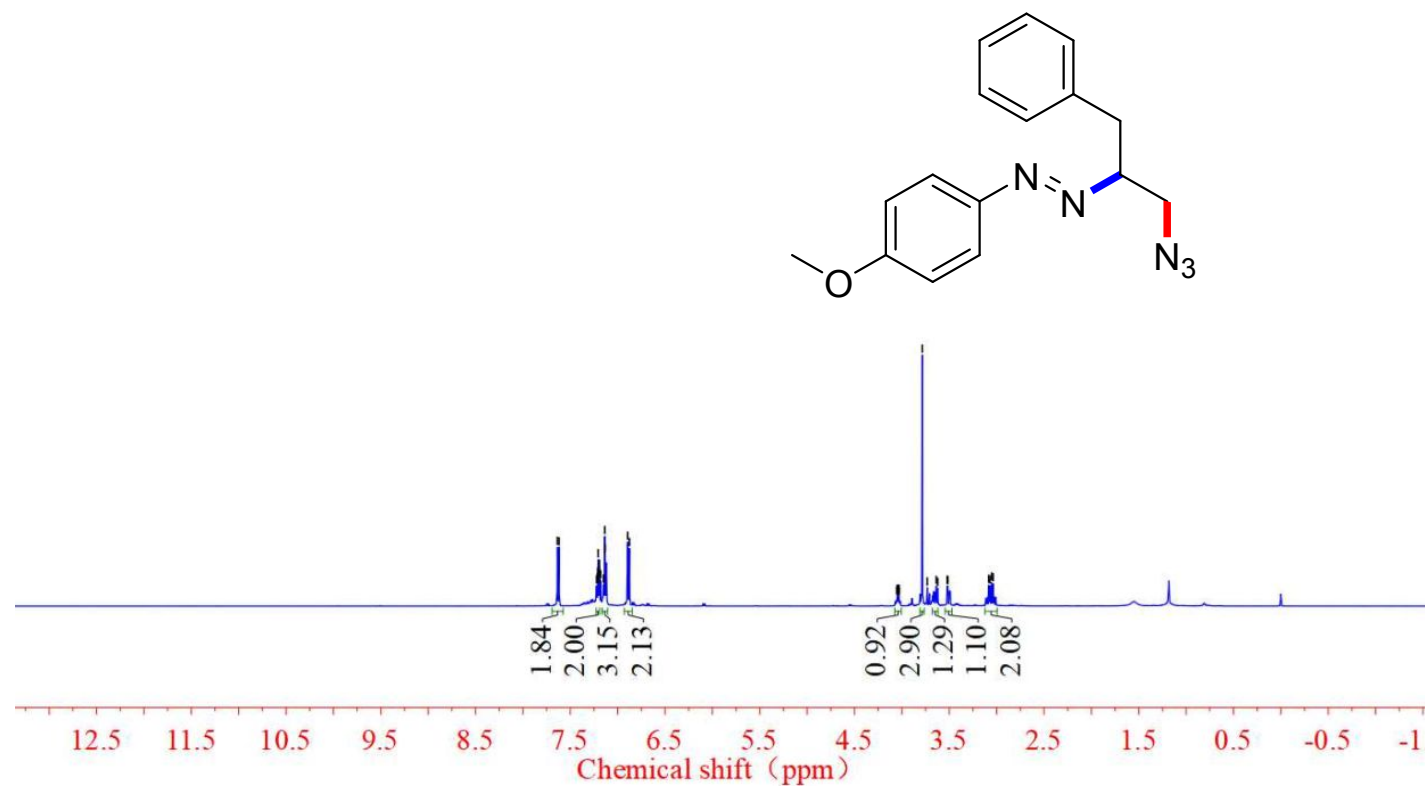

4f ${ }^{13}$ C NMR (126 MHz, Chloroform-d)

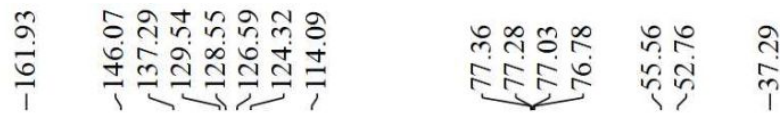
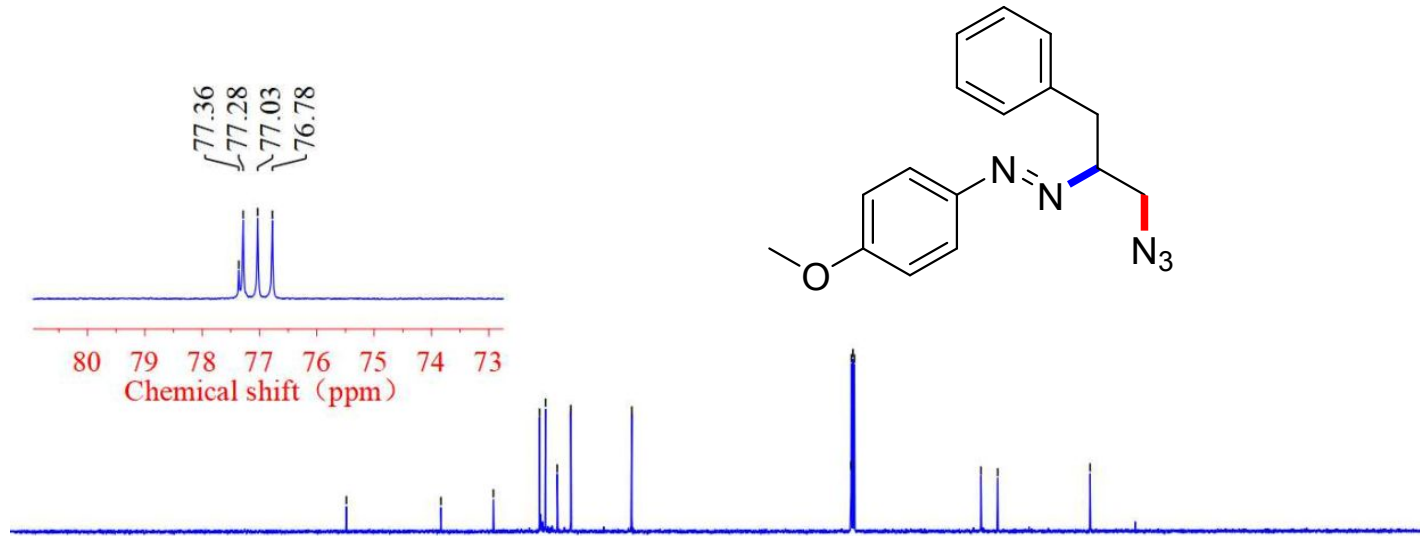

$\begin{array}{rlllllllllllllllllllllll}210 & 200 & 190 & 180 & 170 & 160 & 150 & 140 & 130 & 120 & 110 & 100 & 90 & 80 & 70 & 60 & 50 & 40 & 30 & 20 & 10 & 0 & -10 \\ \text { Chemical shift } & (\mathrm{ppm})\end{array}$ 


\section{4g ${ }^{1}$ H NMR (500 MHz, Chloroform-d)}

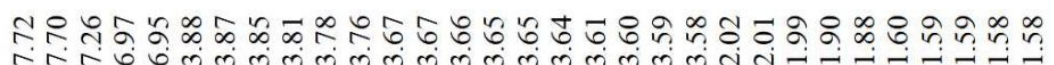
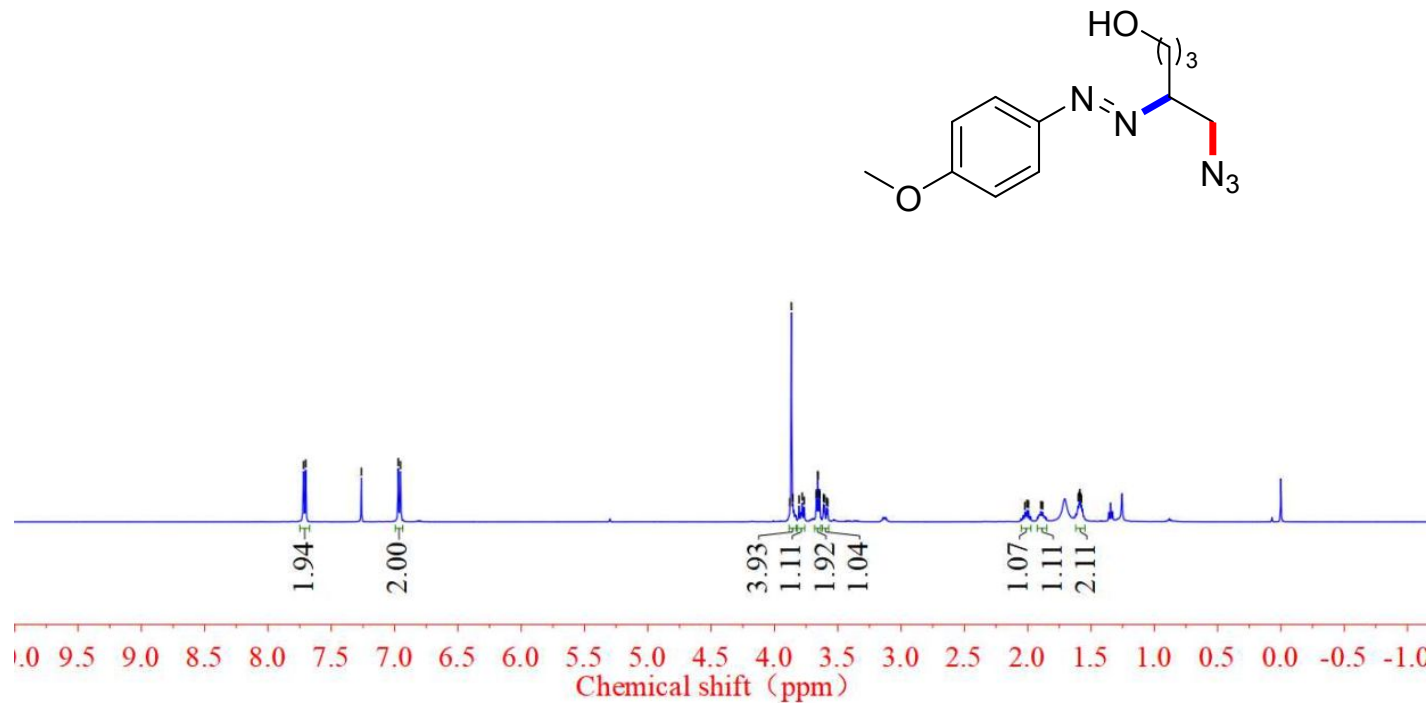

4g ${ }^{13} \mathrm{C}$ NMR (126 MHz, Chloroform-d)

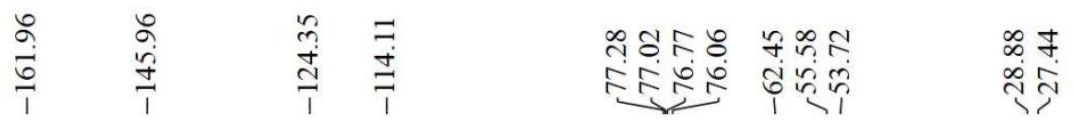

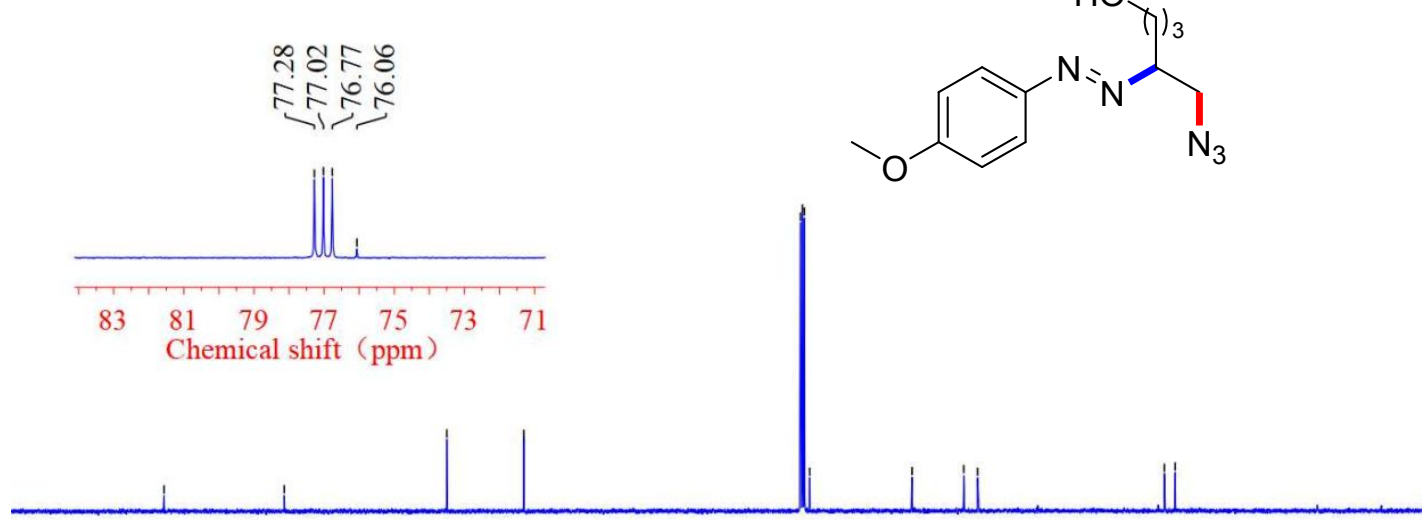

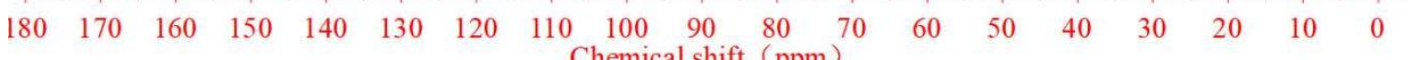




\section{4h ${ }^{1} \mathrm{H}$ NMR (500 MHz, Chloroform-d)}

吾

œ

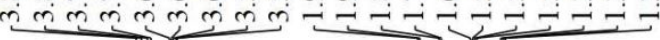
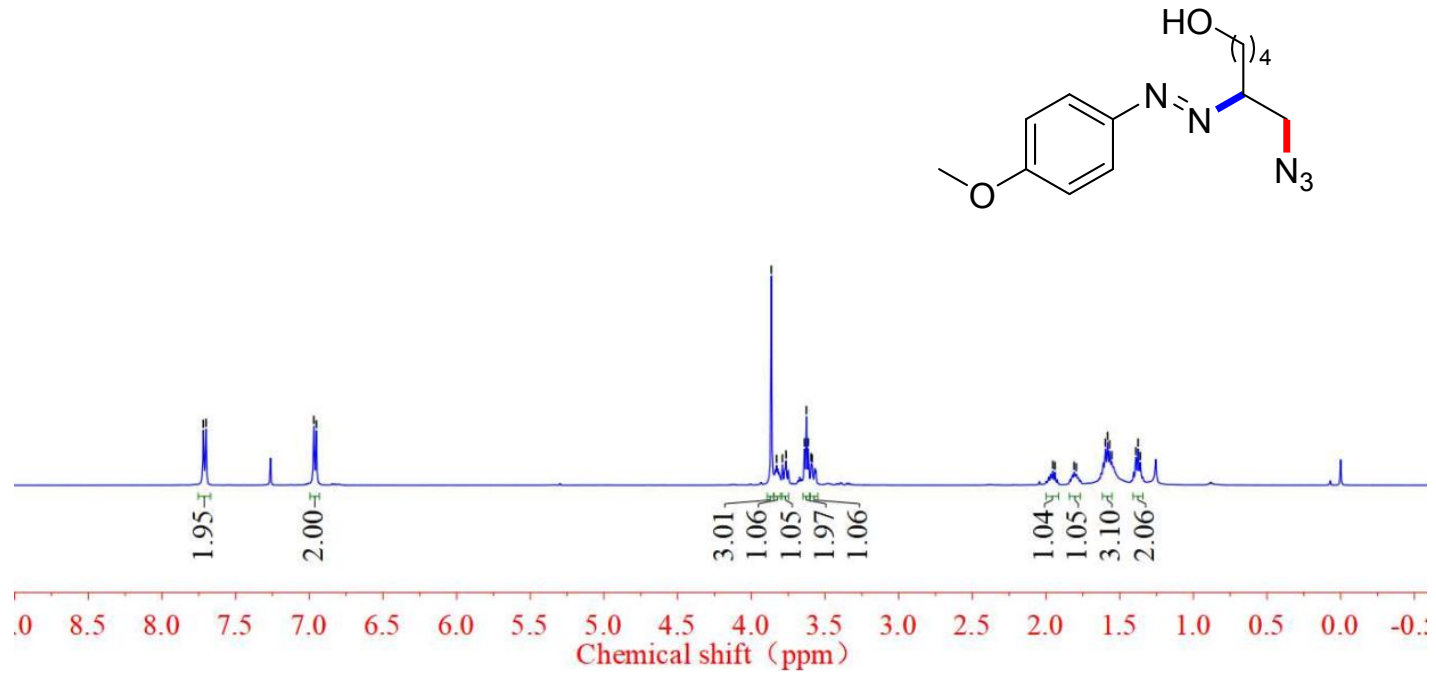

\section{4h ${ }^{13}$ C NMR (126 MHz, Chloroform-d)}

\begin{tabular}{|c|c|c|}
\hline \begin{tabular}{l} 
Oै \\
0 \\
\multirow{1}{*}{} \\
\end{tabular} & $\begin{array}{ll}\vec{m} & g \\
\underset{1}{+} & \Xi\end{array}$ & 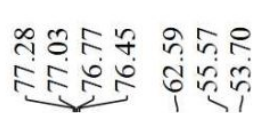 \\
\hline
\end{tabular}

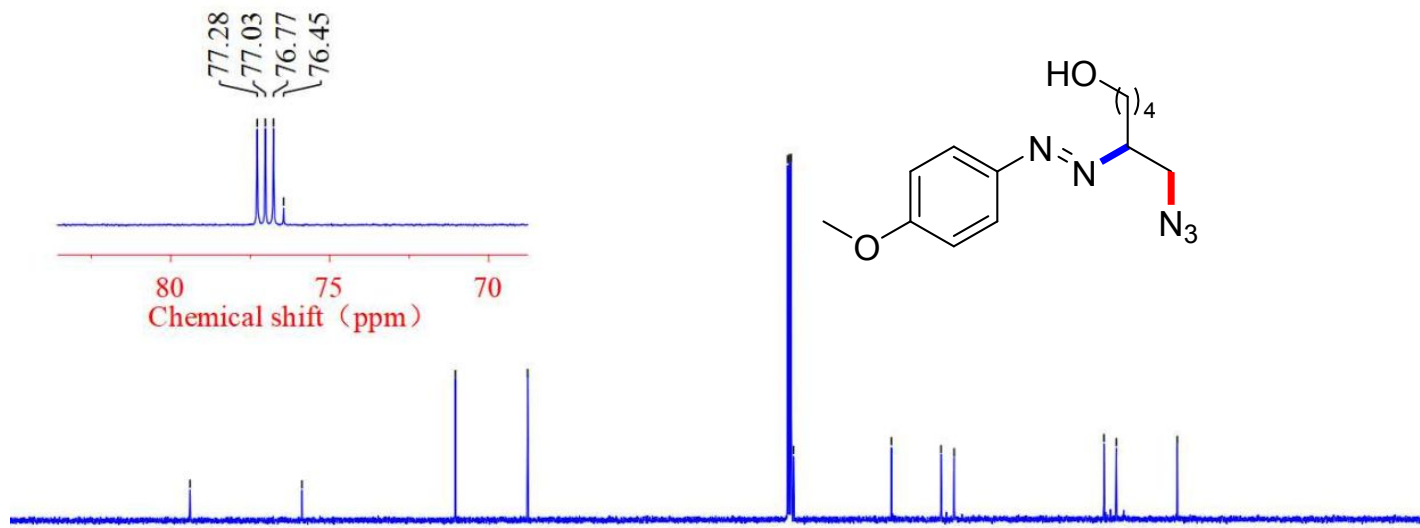

$\begin{array}{llllllllllllllllllll}180 & 170 & 160 & 150 & 140 & 130 & 120 & 110 & 100 & 90 & 80 & 70 & 60 & 50 & 40 & 30 & 20 & 10 & 0 & -10\end{array}$ 


\section{4i ${ }^{1}$ H NMR (500 MHz, Chloroform-d)}

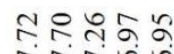

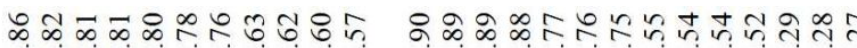

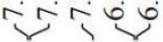

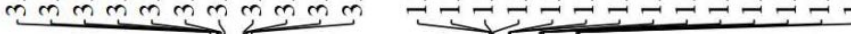

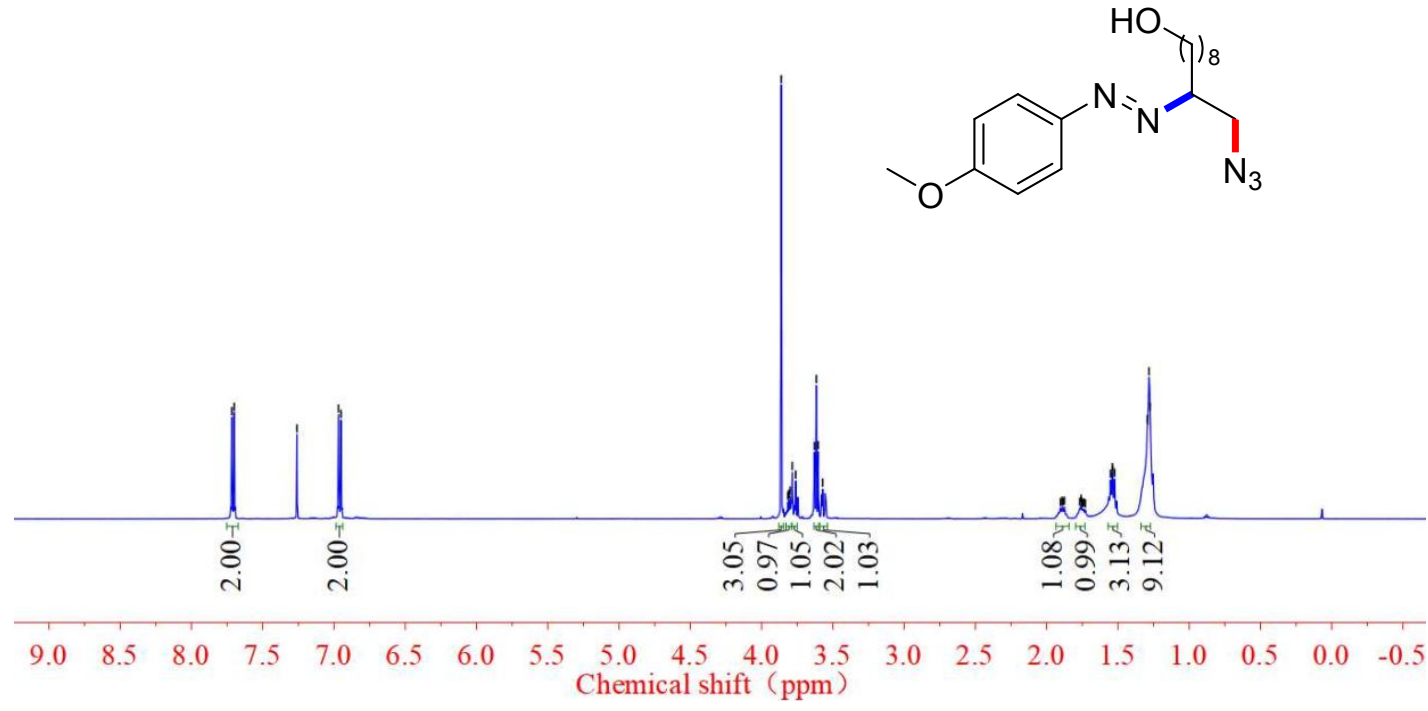

4i ${ }^{13} \mathrm{C}$ NMR (126 MHz, Chloroform-d)

\begin{tabular}{|c|c|c|c|}
\hline $\begin{array}{l}\infty \\
0 \\
0 \\
+1 \\
1\end{array}$ & 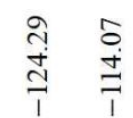 & 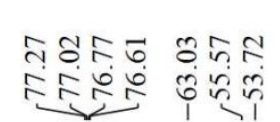 & 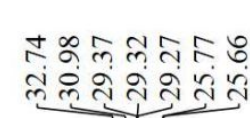 \\
\hline
\end{tabular}
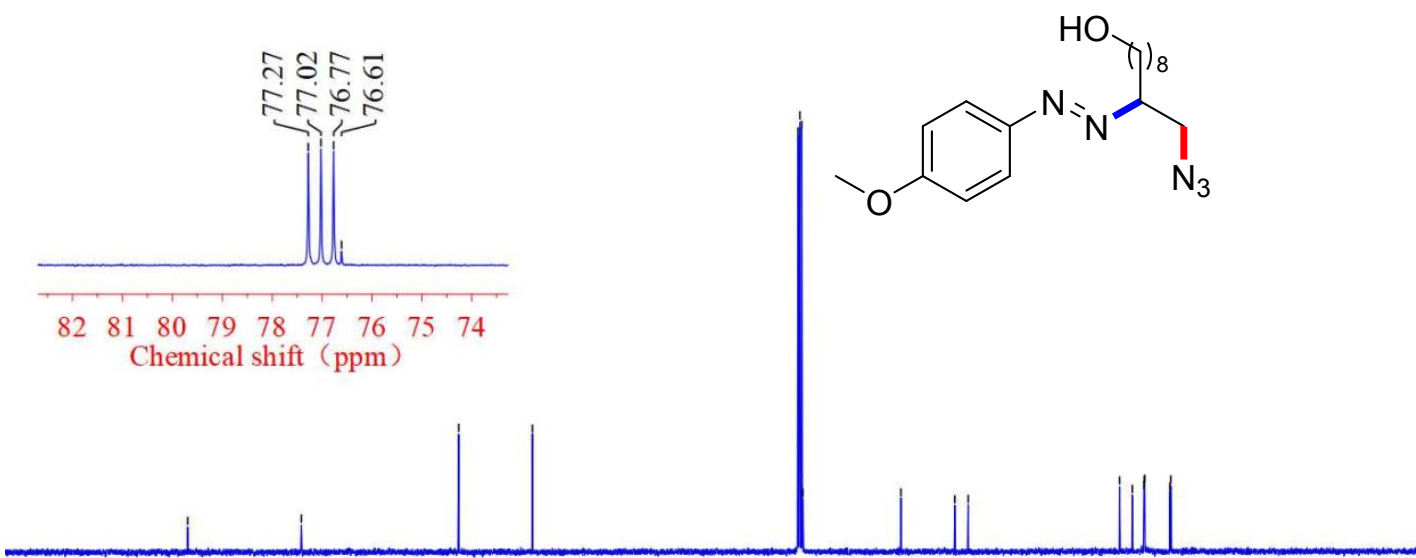

$\begin{array}{llllllllllllllllll}180 & 170 & 160 & 150 & 140 & 130 & 120 & 110 & 100 & \begin{array}{l}90 \\ \text { Chemical shift }\end{array}\left(\begin{array}{ll}80 \\ (\mathrm{ppm})\end{array}\right.\end{array}$ 


\section{$4 \mathrm{j}^{1} \mathrm{H}$ NMR (500 MHz, Chloroform- $d$ )}

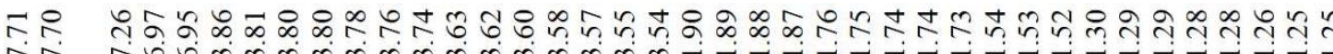

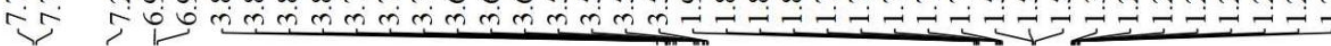

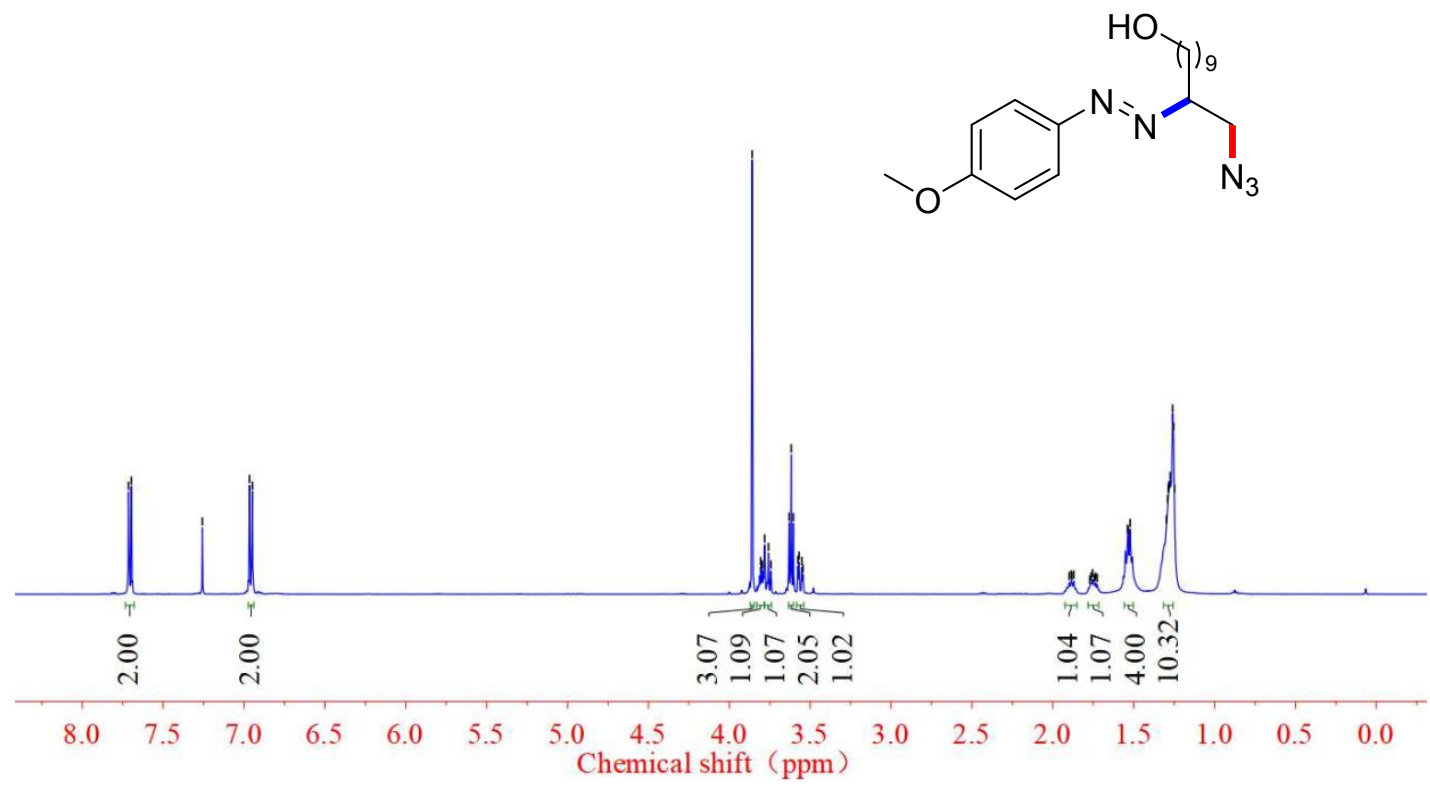

\section{4j ${ }^{13} \mathrm{C}$ NMR (126 MHz, Chloroform- $d$ )}

\begin{tabular}{|c|c|c|c|c|}
\hline $\begin{array}{l}\text { oे } \\
\text { ¿े } \\
\text { I }\end{array}$ & 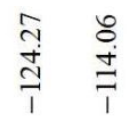 & 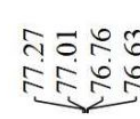 & 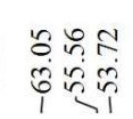 & 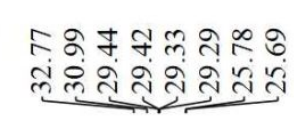 \\
\hline
\end{tabular}

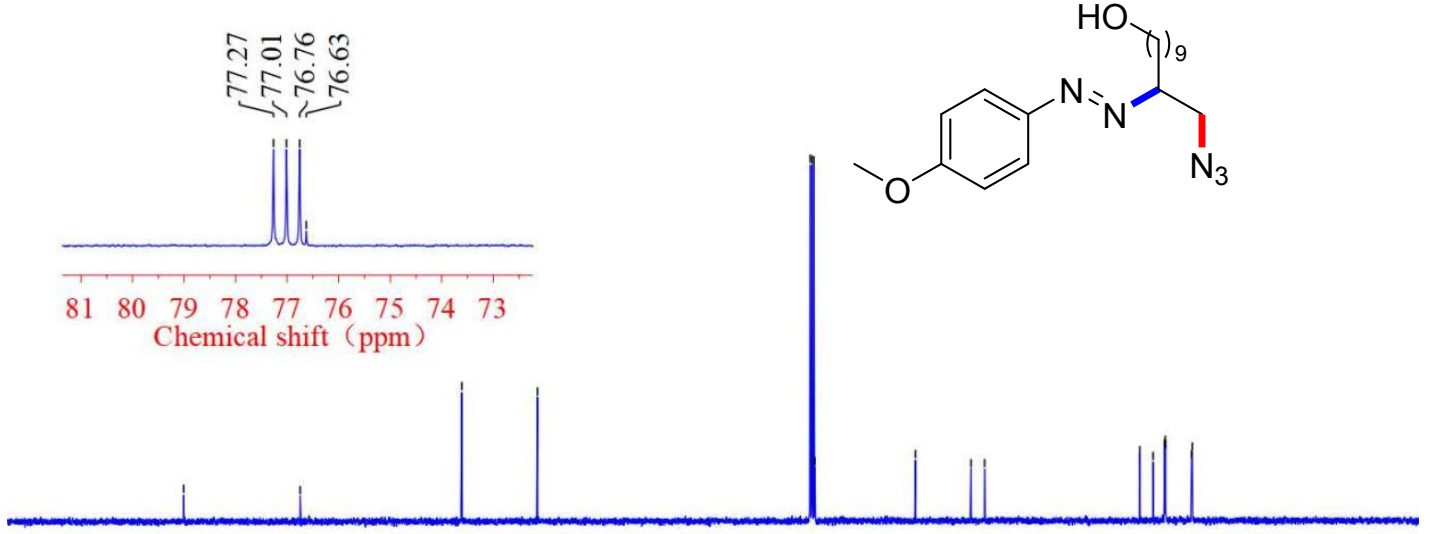

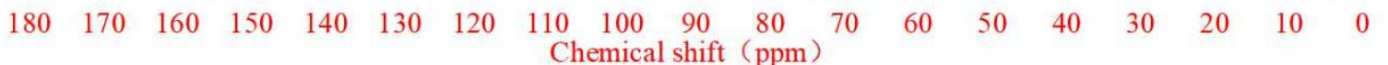




\section{4k ${ }^{1} \mathrm{H}$ NMR (500 MHz, Chloroform-d)}

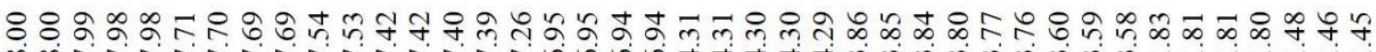

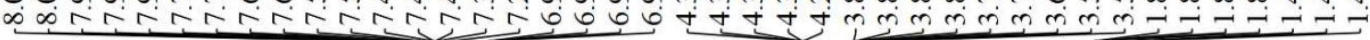

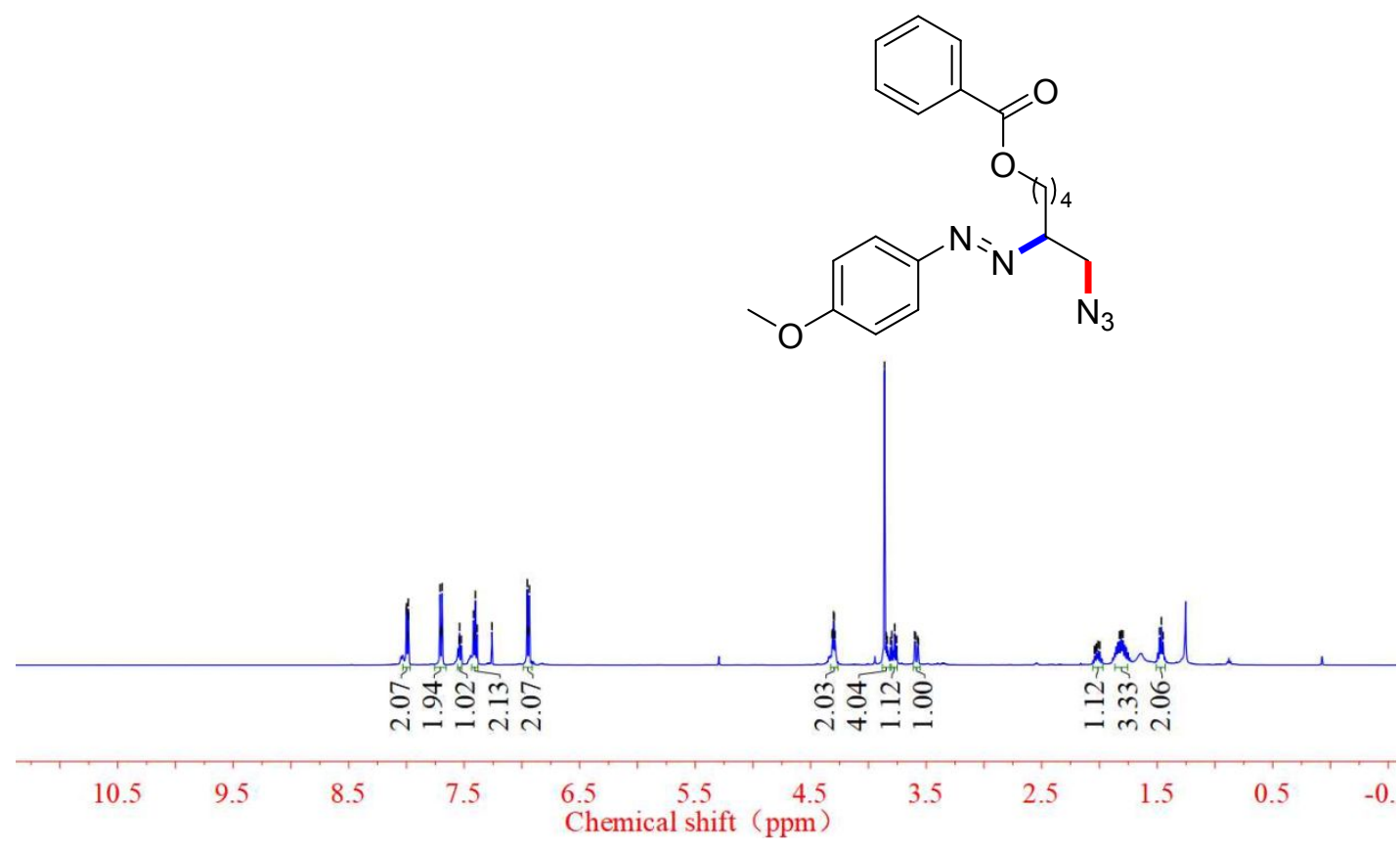

\section{4k ${ }^{13}$ C NMR (126 MHz, Chloroform-d)}

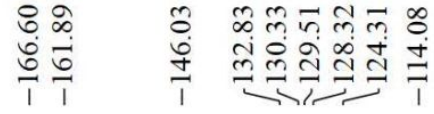

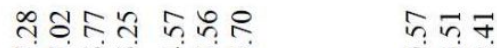

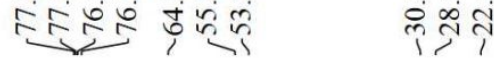
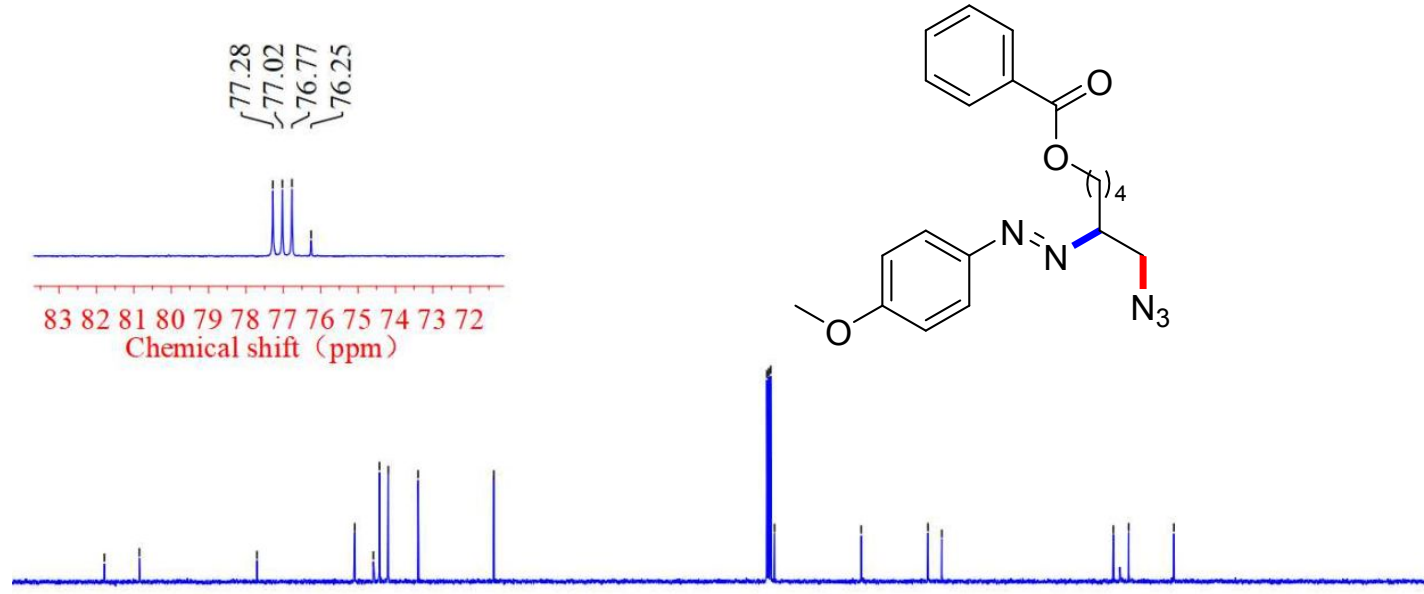

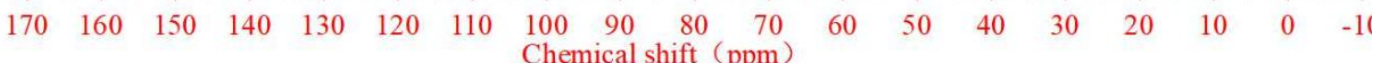




\section{$4{ }^{1} \mathrm{H}$ NMR (500 MHz, Chloroform-d)}

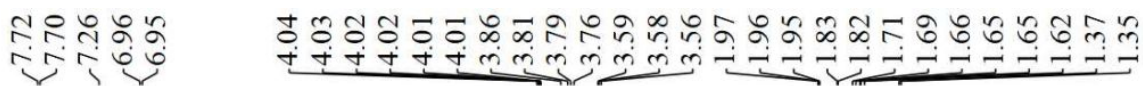

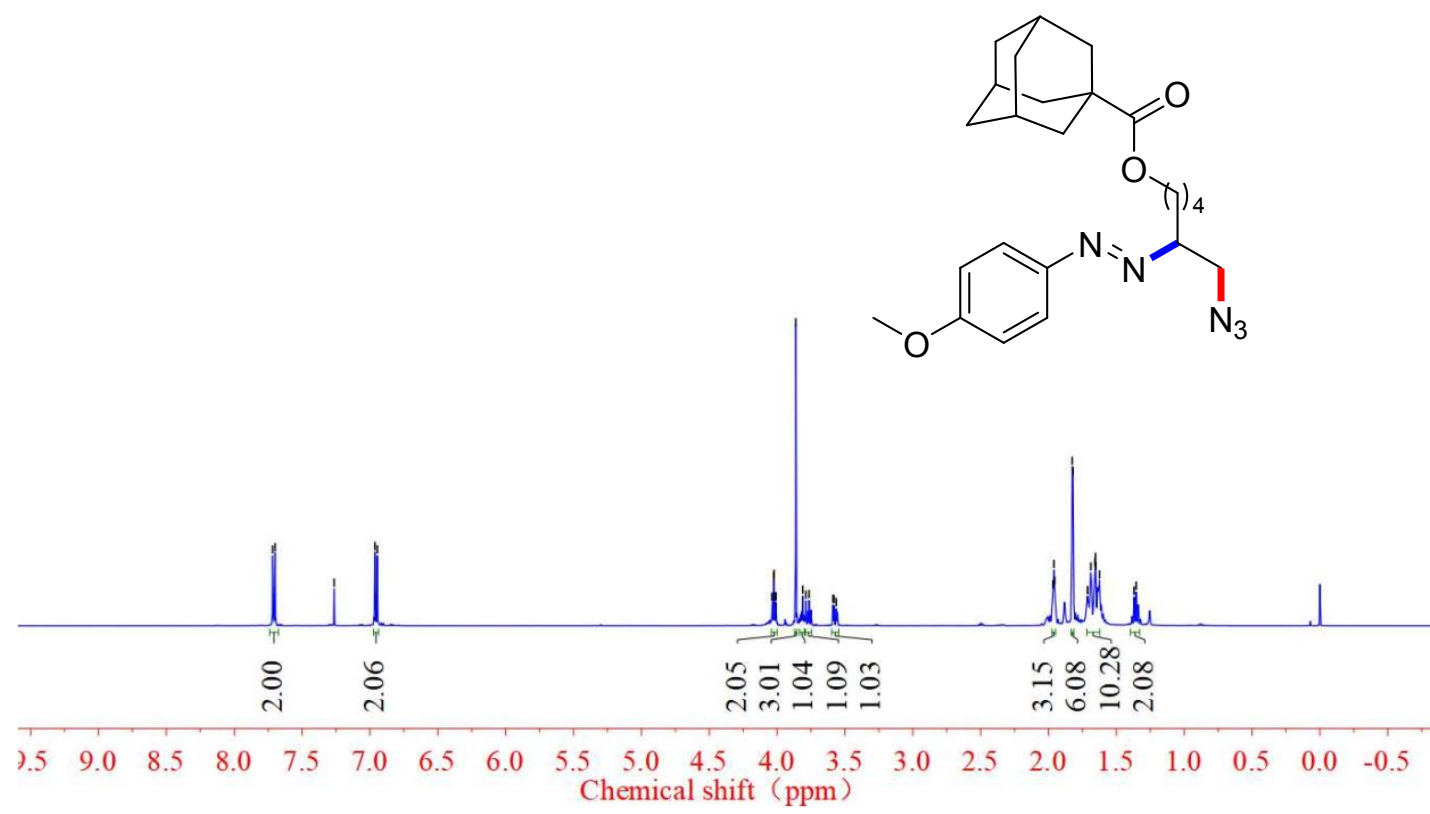

\section{$4{ }^{13}$ C NMR (126 MHz, Chloroform-d)}

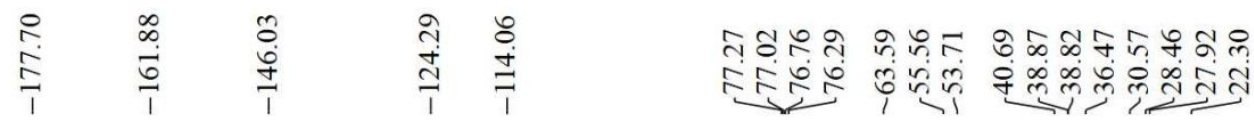

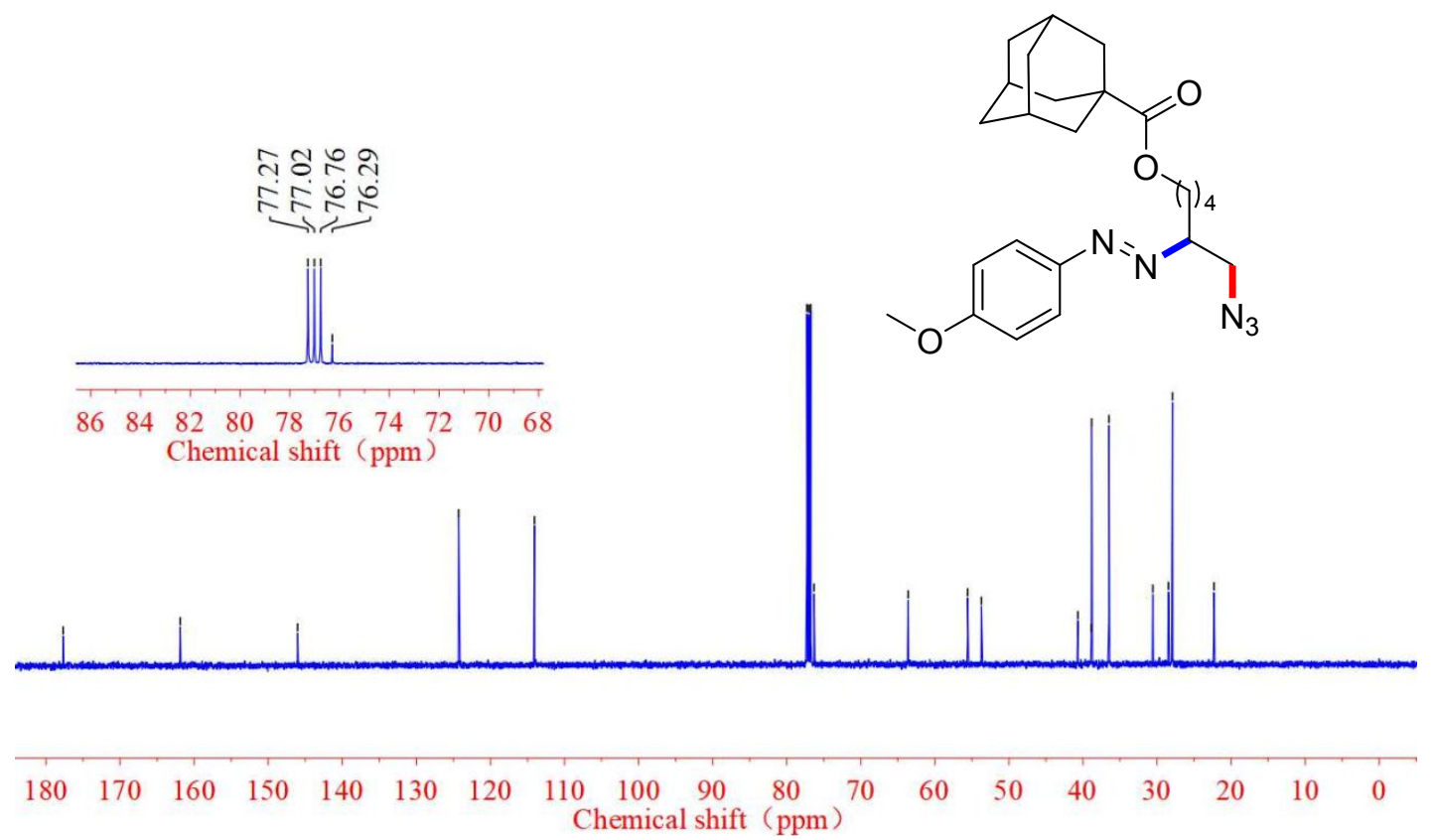




\section{4m ${ }^{1} \mathrm{H}$ NMR (500 MHz, Chloroform-d)}

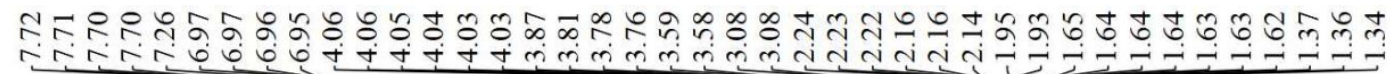

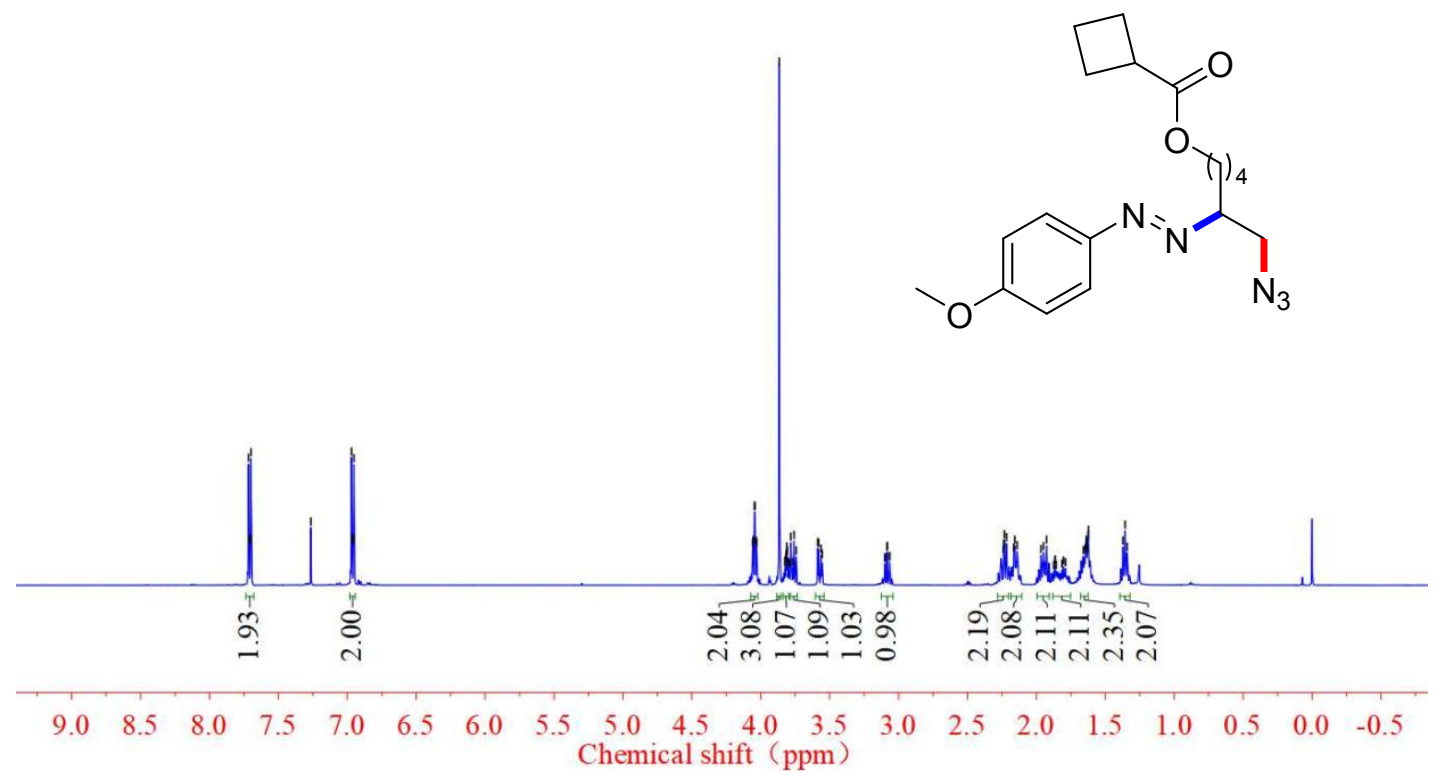

4m ${ }^{13}$ C NMR (126 MHz, Chloroform-d)

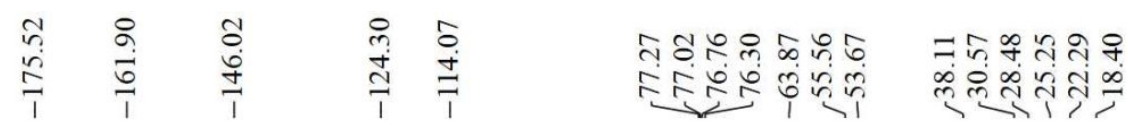
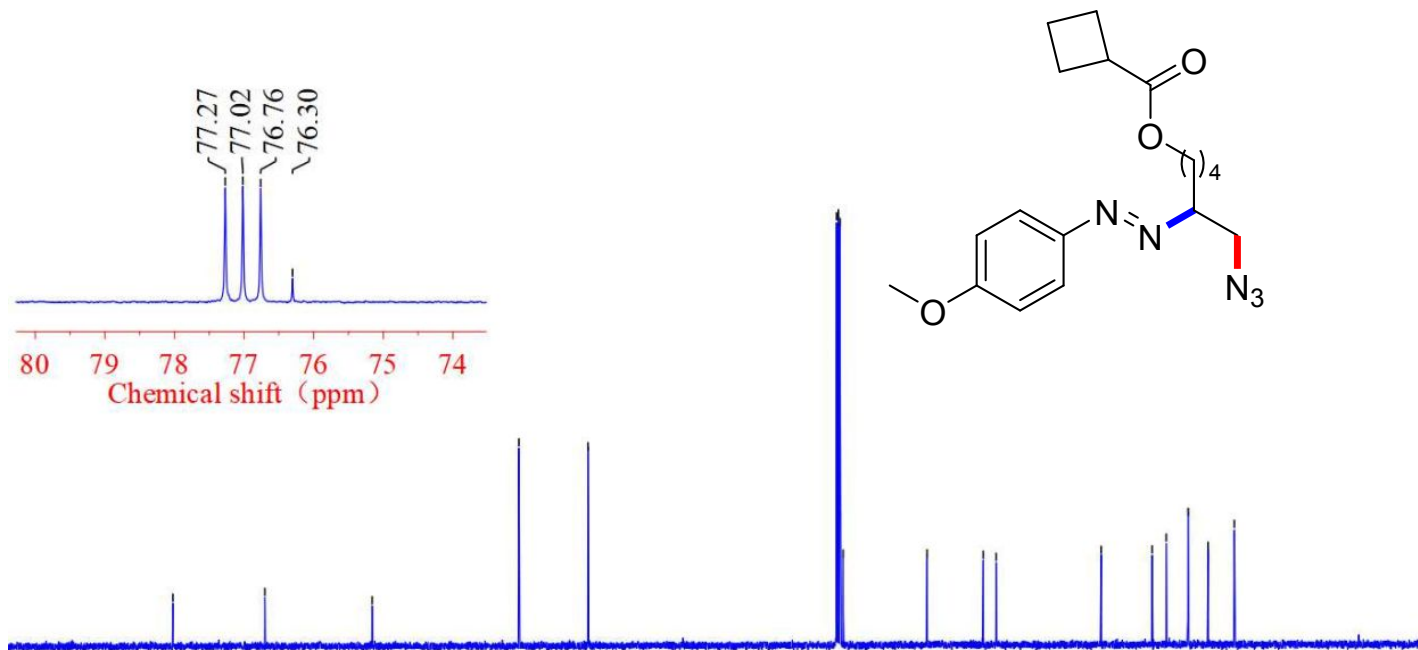

$\begin{array}{lllllllllllllllllll}190 & 180 & 170 & 160 & 150 & 140 & 130 & 120 & \left.\begin{array}{c}110 \\ \text { Chemical shift }\end{array} \text { (ppm }\right)^{100} & 90 & 80 & 70 & 60 & 50 & 40 & 30 & 20 & 10 & 0\end{array}$ 


\section{4n ${ }^{1}$ H NMR (500 MHz, Chloroform-d)}

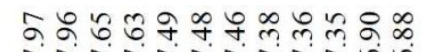

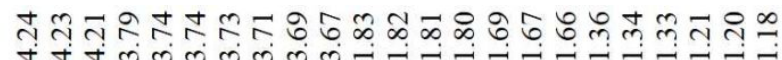

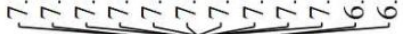

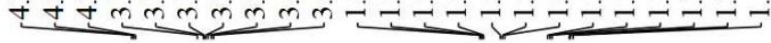

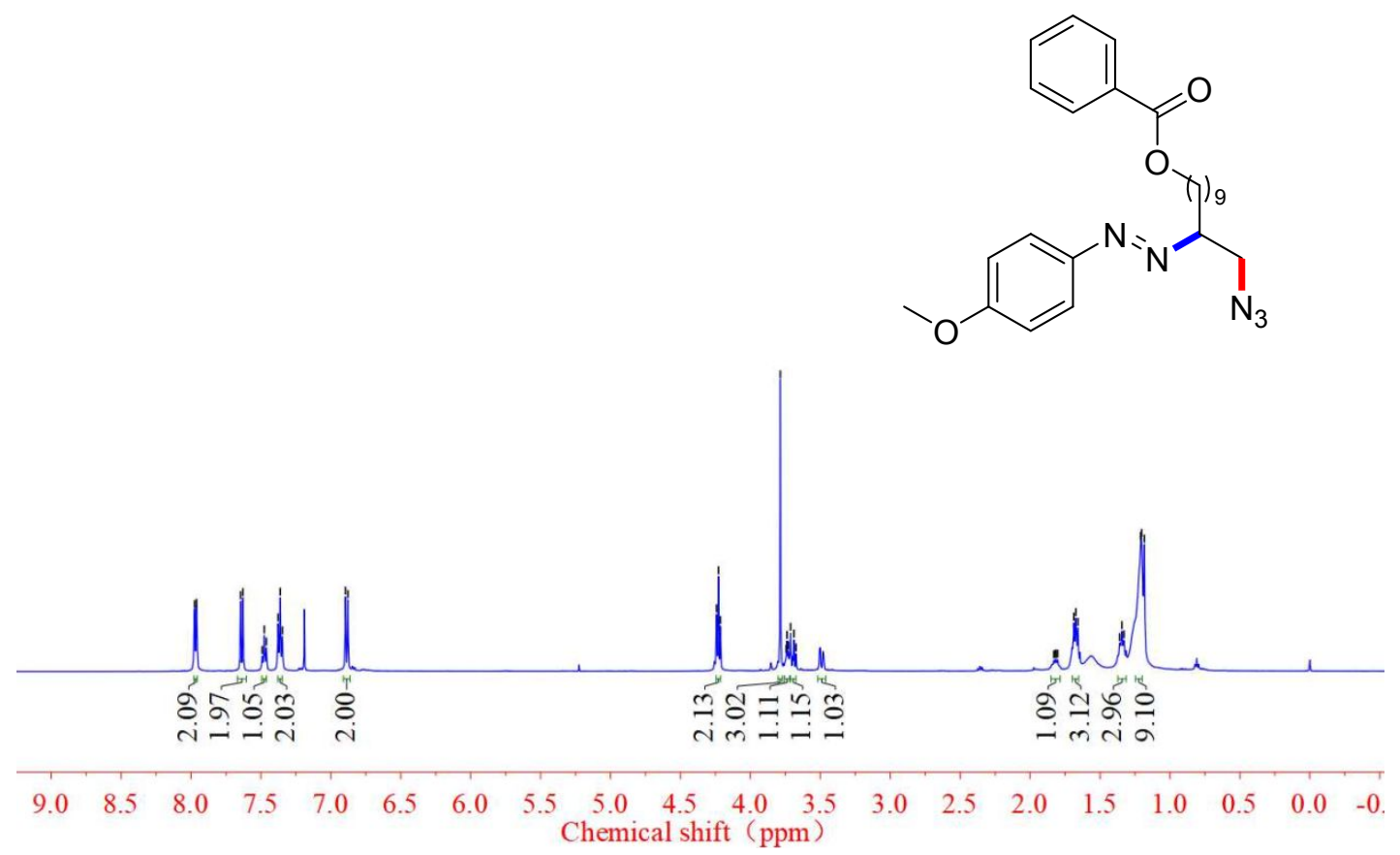

4n ${ }^{13} \mathrm{C}$ NMR (126 MHz, Chloroform-d)

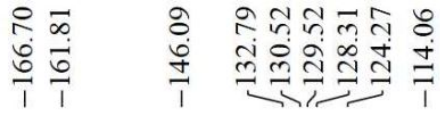

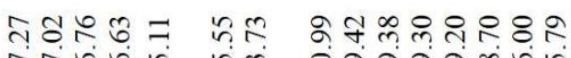

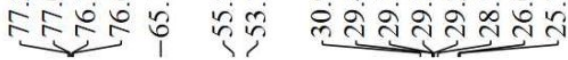
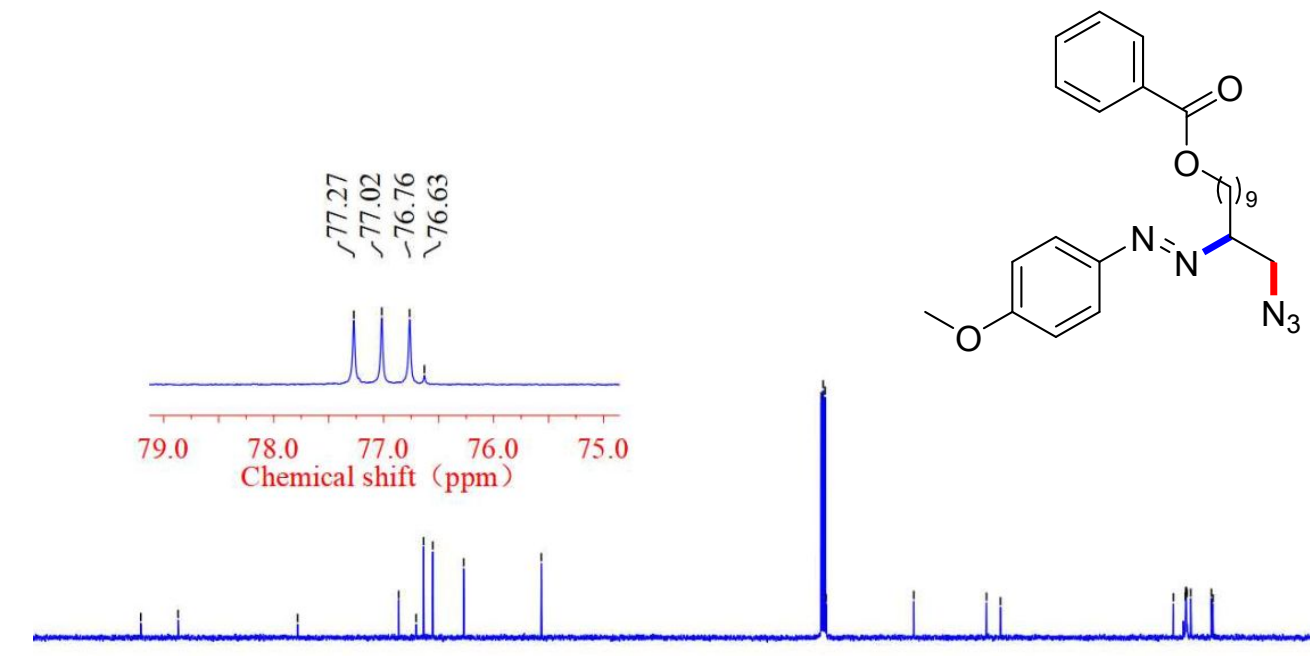

$\begin{array}{llllllllllllllllll}80 & 170 & 160 & 150 & 140 & 130 & 120 & 110 & \begin{array}{c}100 \\ \text { Chemical shift }\end{array}(\mathrm{ppm})\end{array}$ 


\section{$40^{1}$ H NMR (500 MHz, Chloroform-d)}

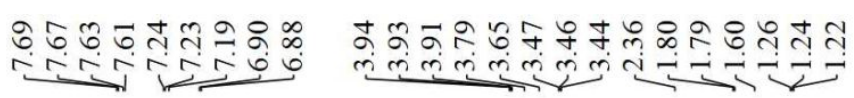
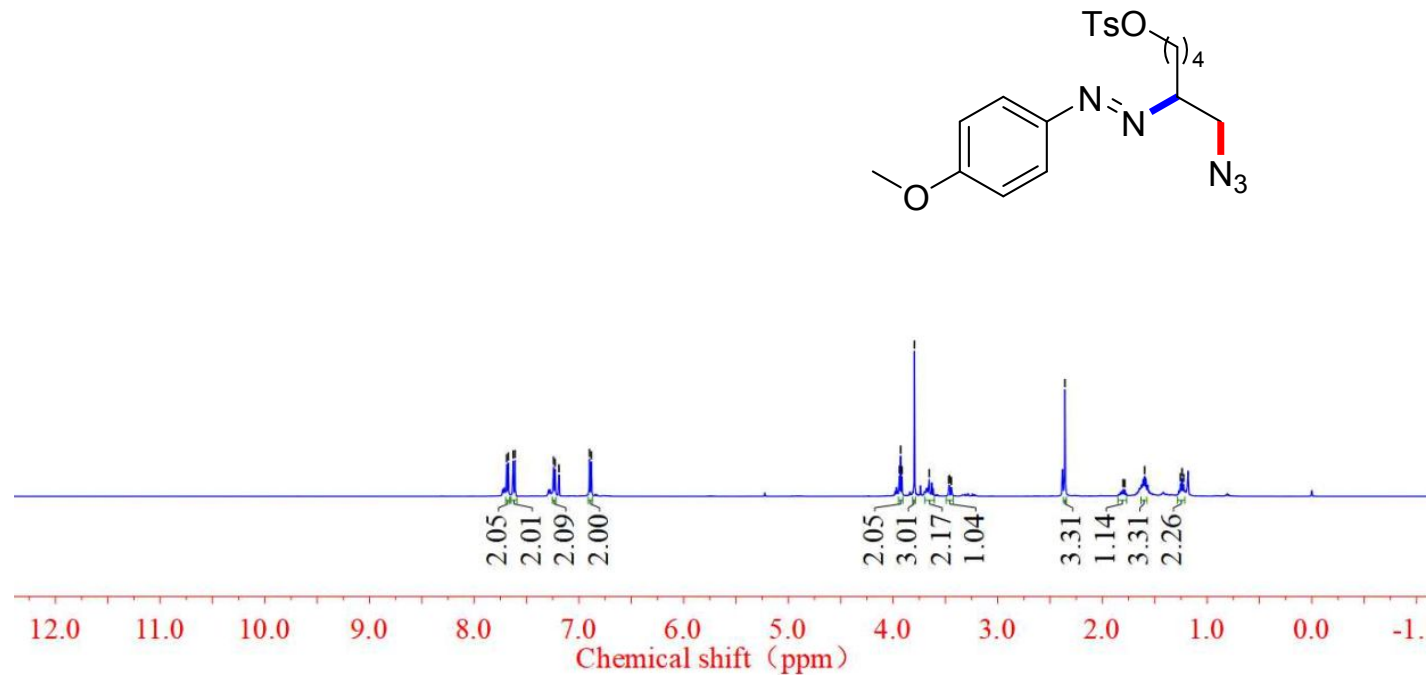

$40{ }^{13} \mathrm{C}$ NMR (126 MHz, Chloroform-d)

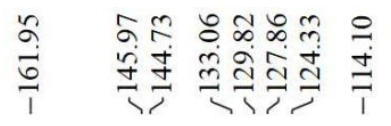

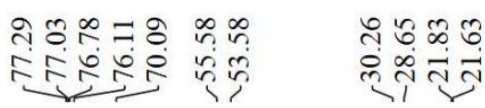
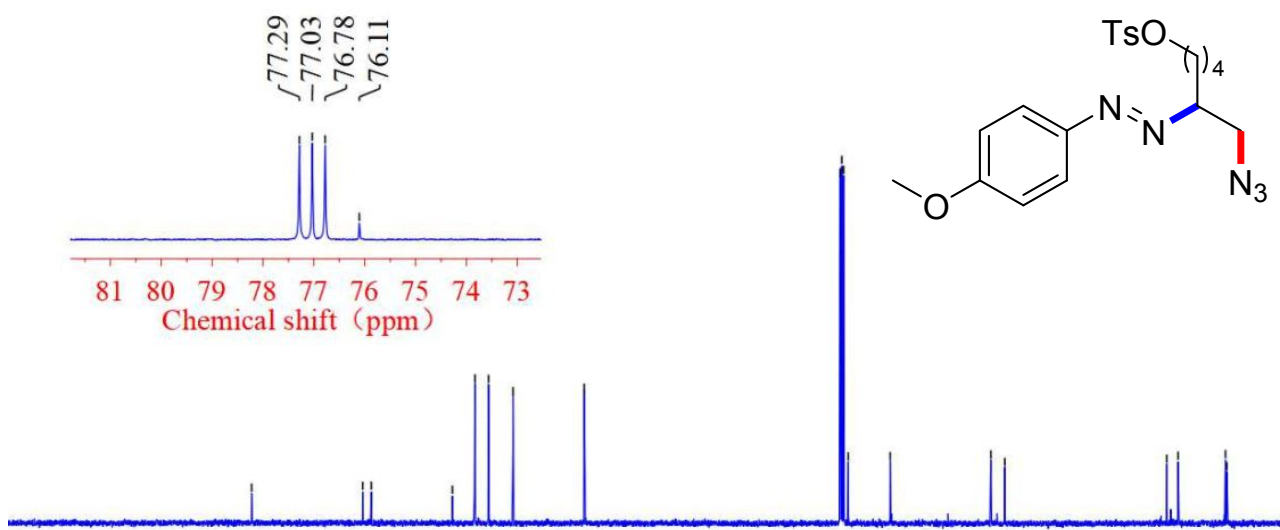

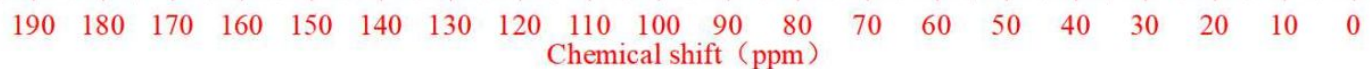




\section{4p ${ }^{1} \mathrm{H}$ NMR (500 MHz, Chloroform-d)}

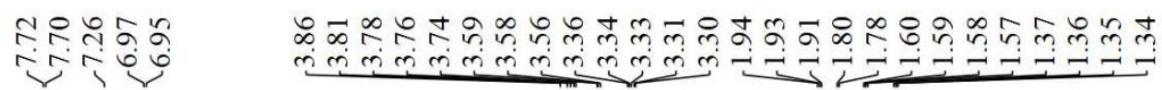

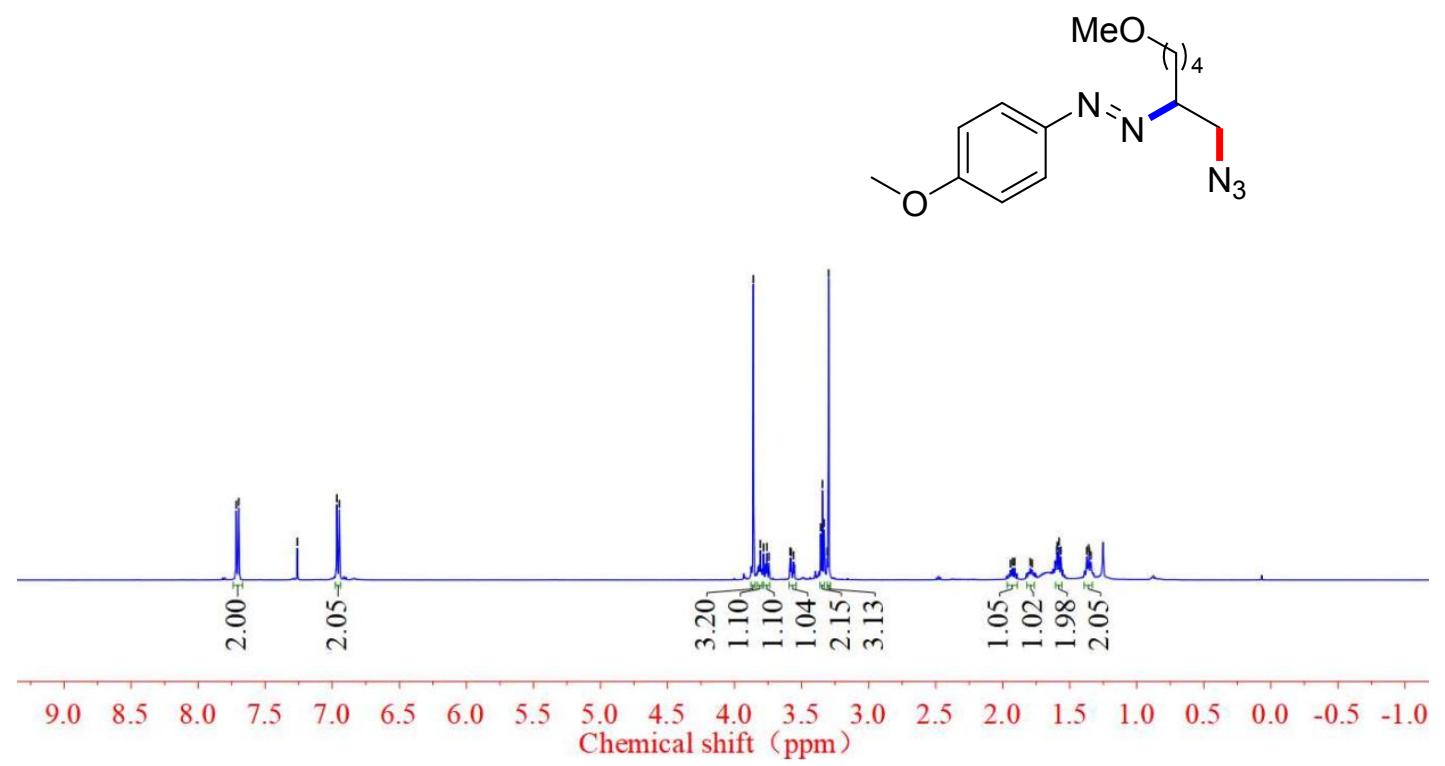

$4 p{ }^{13}$ C NMR (126 MHz, Chloroform-d)

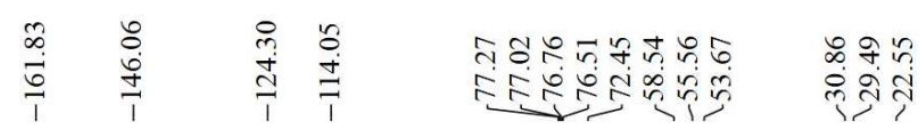
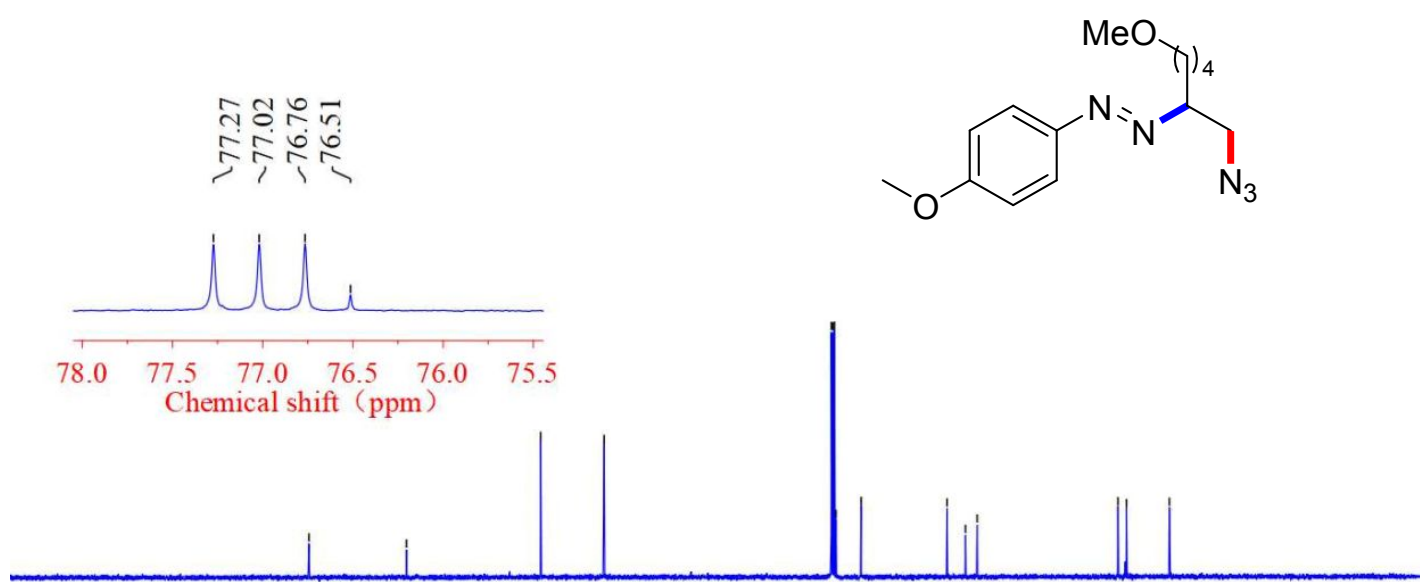

$\begin{array}{lllllllllllllllllllllll}10 & 200 & 190 & 180 & 170 & 160 & 150 & 140 & 130 & 120 & 110 & 100 & 90 & 80 & 70 & 60 & 50 & 40 & 30 & 20 & 10 & 0 & -10 \\ \text { Chemical shift }(\mathrm{ppm})\end{array}$ 


\section{4q ${ }^{1}$ H NMR (500 MHz, Chloroform-d)}

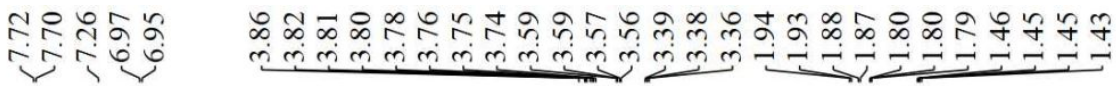<smiles>COc1ccc(N=NC(CN)CBr)cc1</smiles>

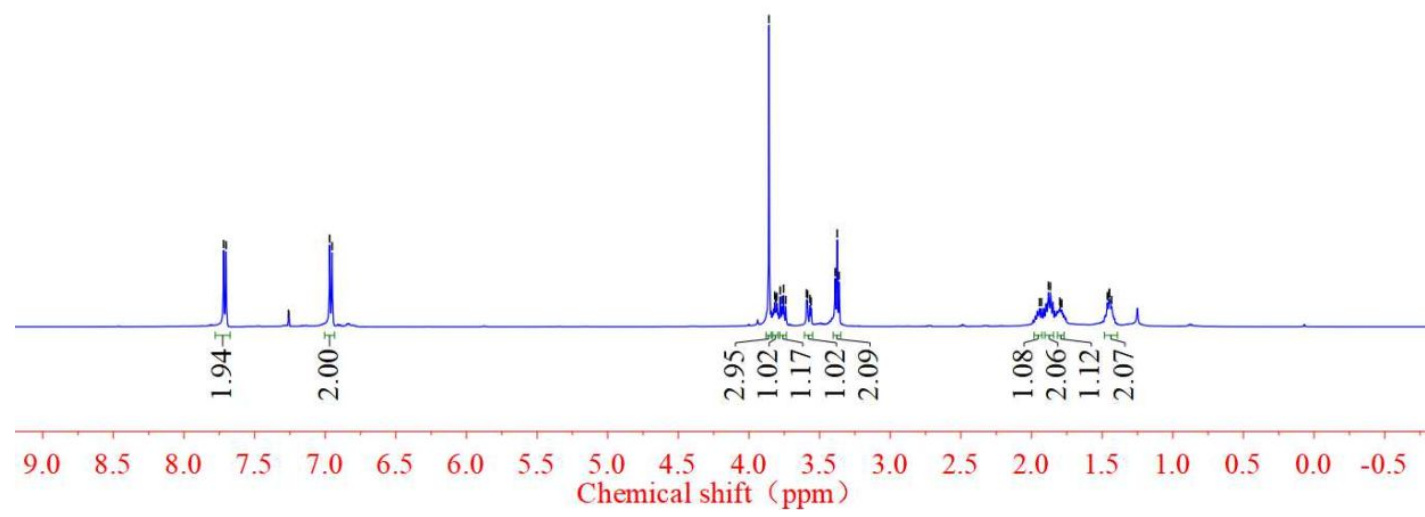

4q ${ }^{13}$ C NMR (126 MHz, Chloroform-d)

\begin{tabular}{|c|c|c|c|}
\hline $\begin{array}{l}8 \\
0 \\
\\
1 \\
1\end{array}$ & 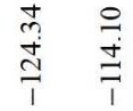 & 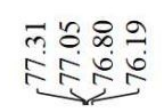 & 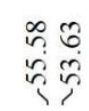 \\
\hline
\end{tabular}
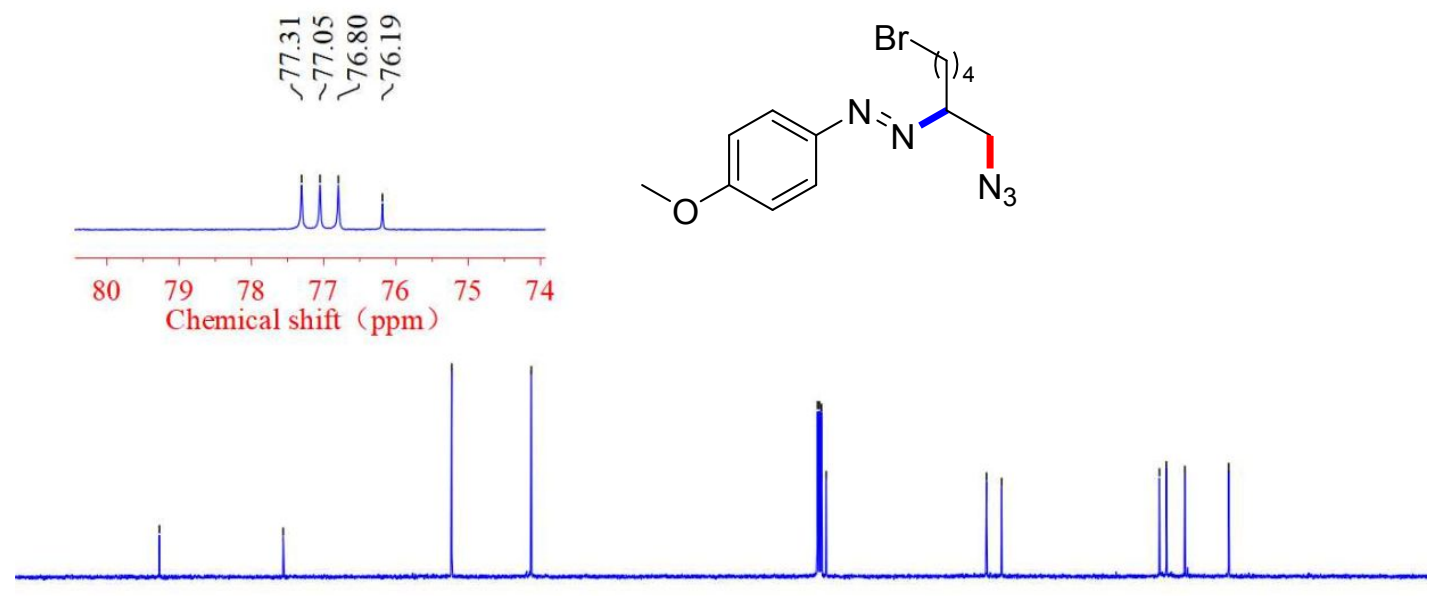

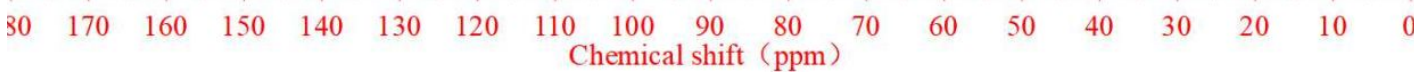




\section{4r ${ }^{1}$ H NMR (500 MHz, Chloroform-d)}

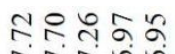

क्ष

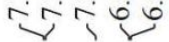<smiles>COc1ccc(N=NC(CBr)CBr)cc1</smiles>

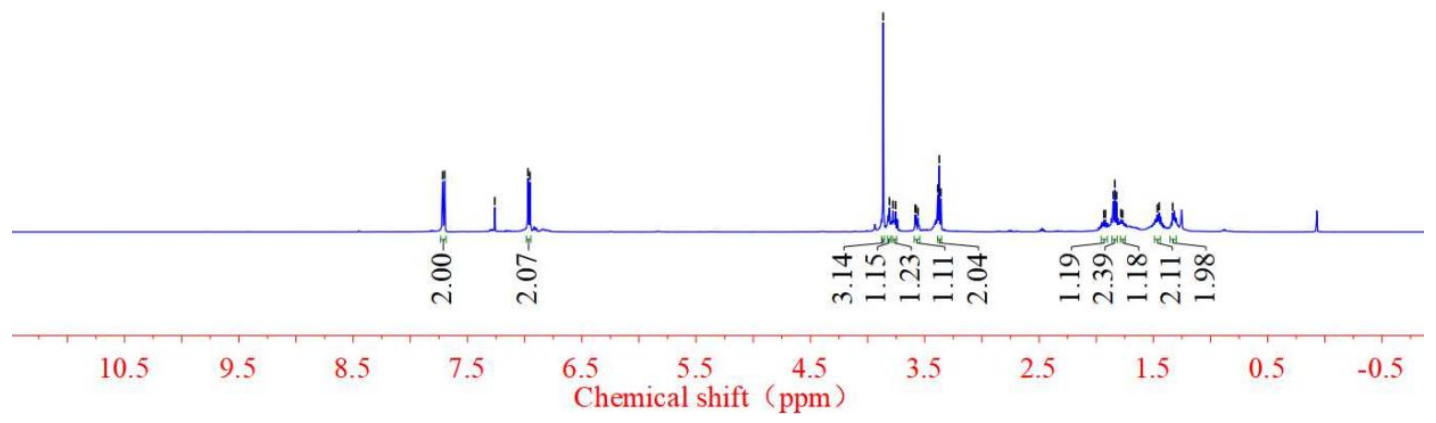

$4 r{ }^{13}$ C NMR (126 MHz, Chloroform-d)

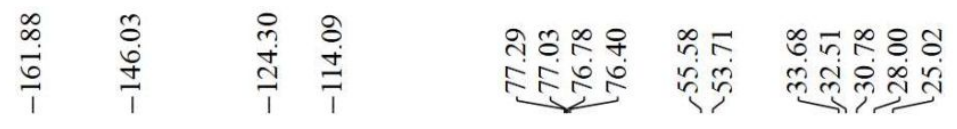

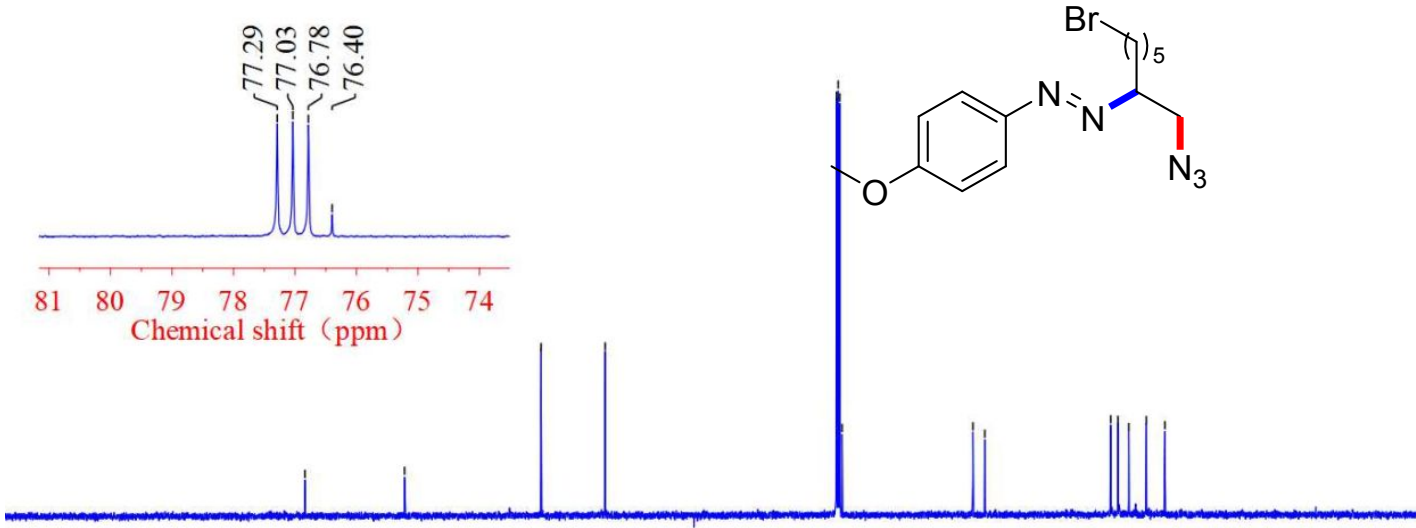

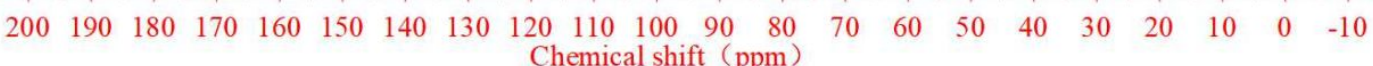




\section{4s ${ }^{1} \mathrm{H}$ NMR (500 MHz, Chloroform-d)}

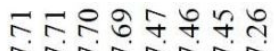

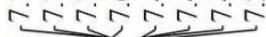

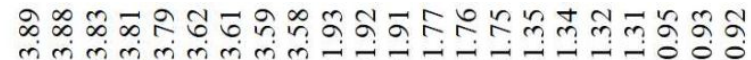

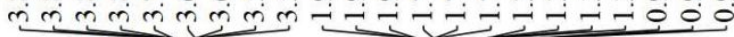<smiles>CCCC(CN)N=Nc1ccccc1</smiles>

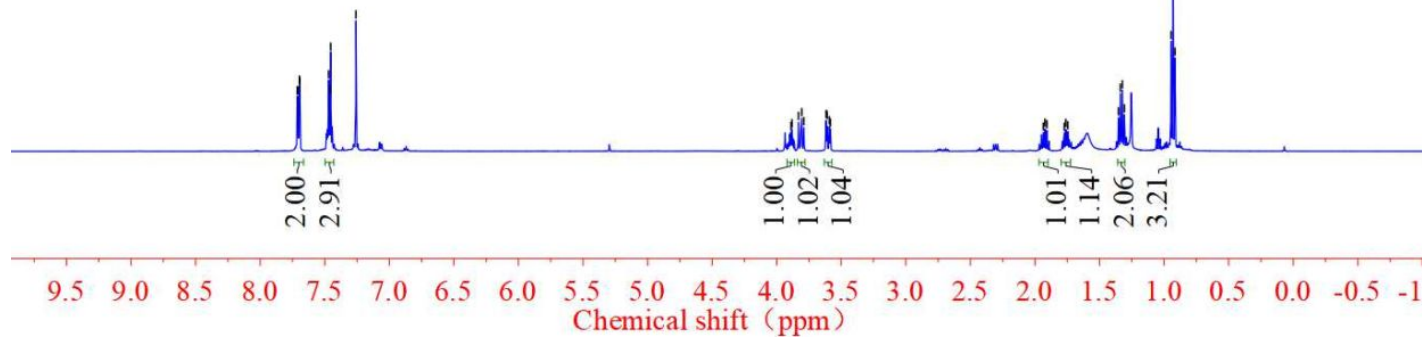

4s ${ }^{13} \mathrm{C}$ NMR (126 MHz, Chloroform-d)

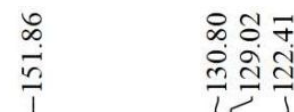

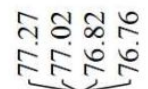

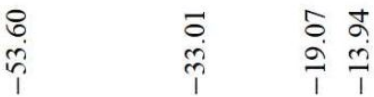

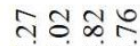

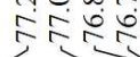<smiles>CC(C)C(CN)N=Nc1ccccc1</smiles>
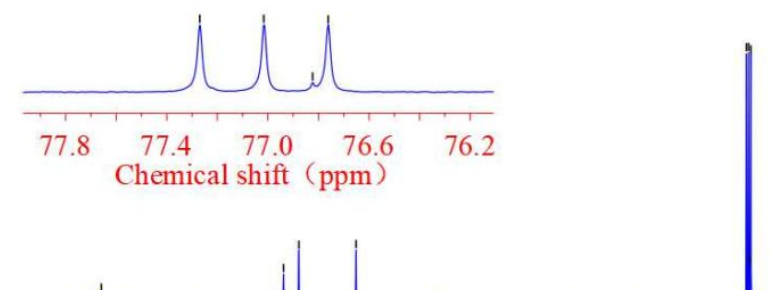


\section{4t ${ }^{1}$ H NMR (500 MHz, Chloroform-d)}

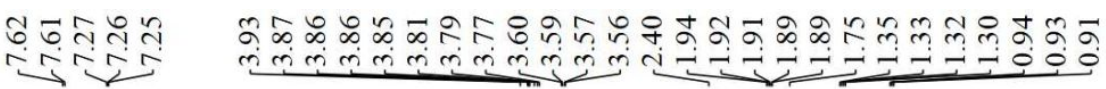

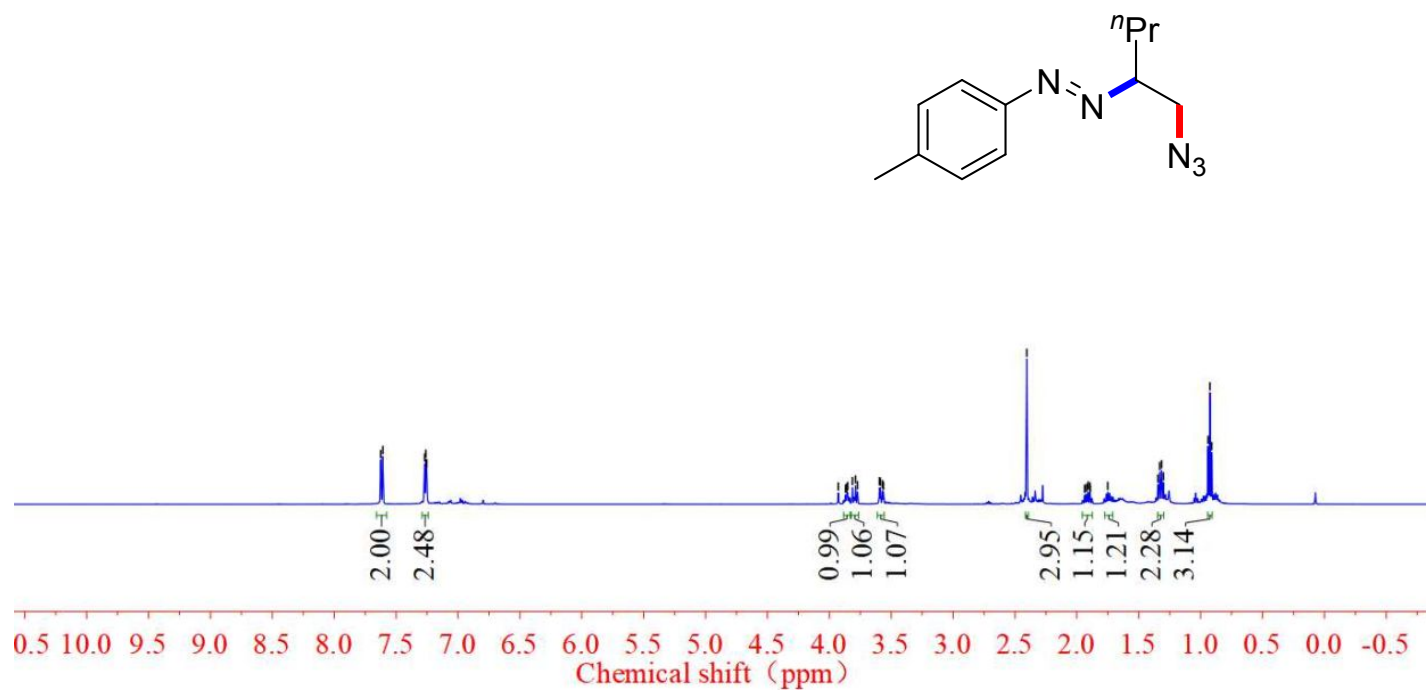

4t ${ }^{13}$ C NMR (126 MHz, Chloroform- $d$ )

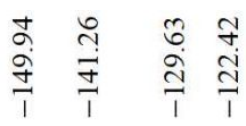

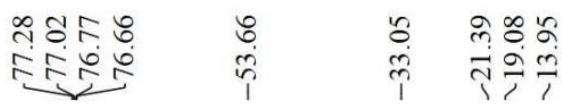
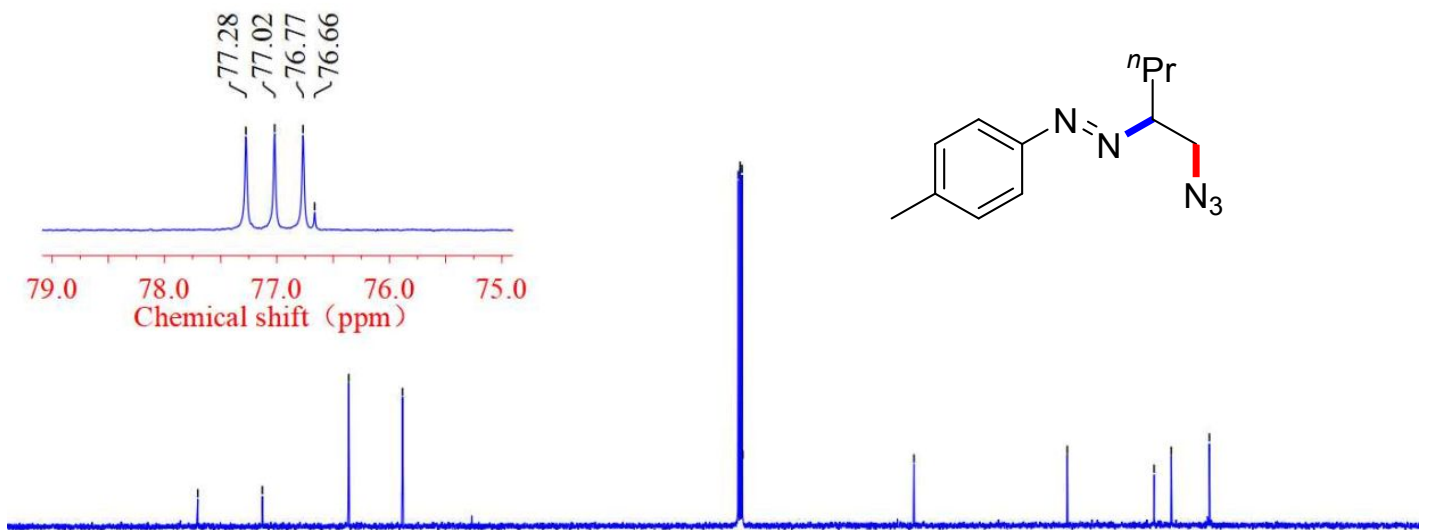

$\begin{array}{lllllllllllllllllll}170 & 160 & 150 & 140 & 130 & 120 & 110 & 100 & \begin{array}{c}90 \\ \text { Chemical shift }\end{array}\left(\begin{array}{c}70 \\ \text { ppm })\end{array}\right. & 60 & 50 & 40 & 30 & 20 & 10 & 0 & -10\end{array}$ 


\section{4u ${ }^{1}$ H NMR (500 MHz, Chloroform-d)}

邹

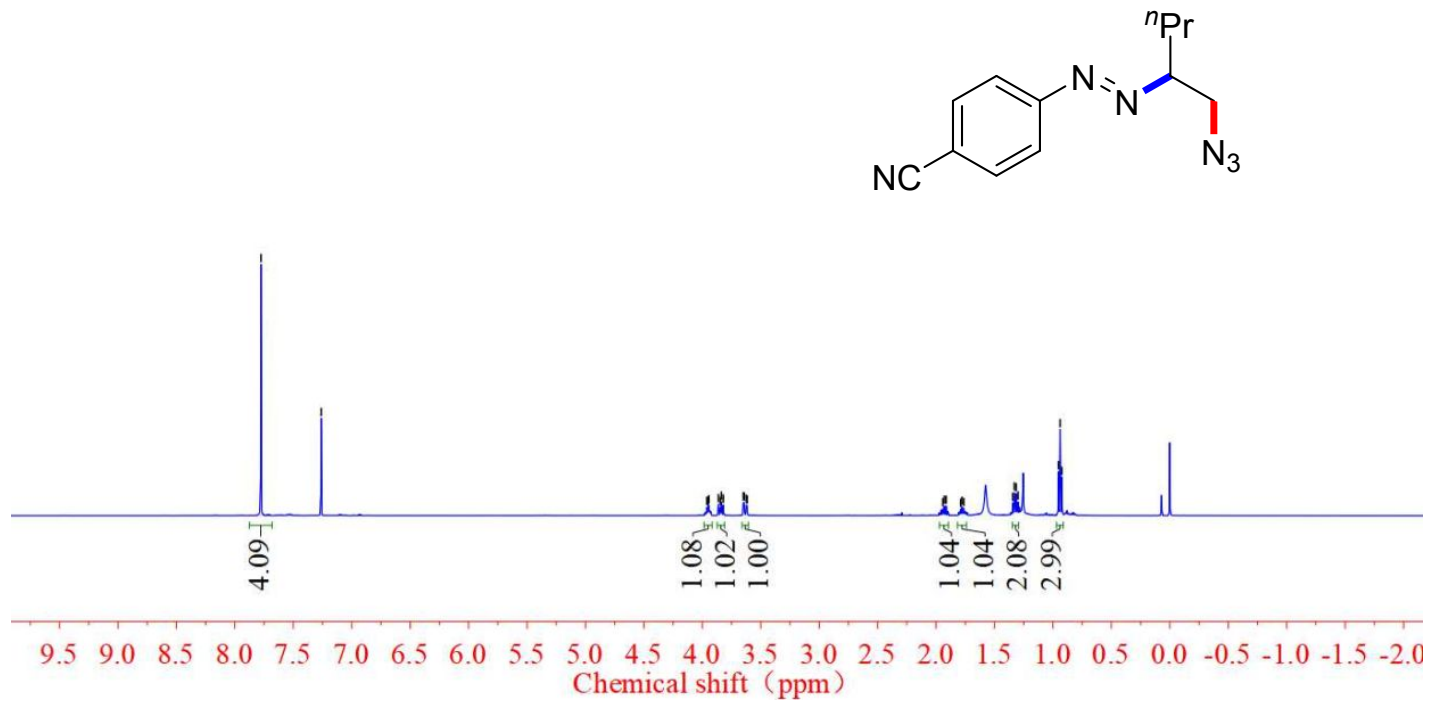

4u ${ }^{13}$ C NMR (126 MHz, Chloroform-d)

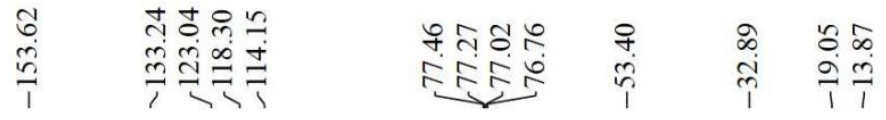
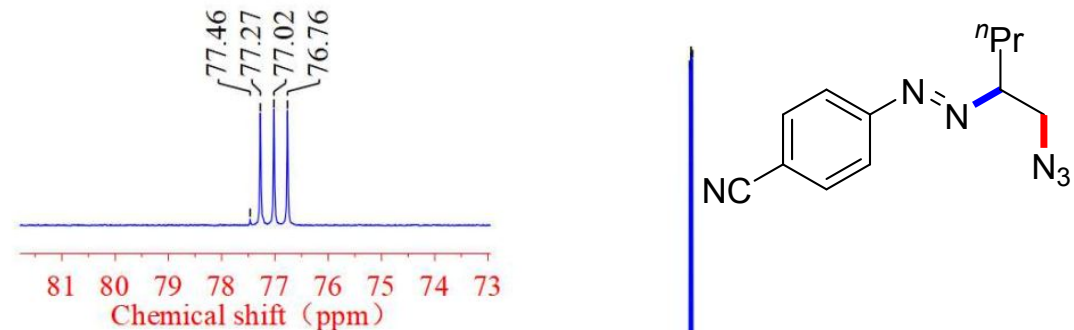
$\begin{array}{rllllllllllllllllllllll}210 & 200 & 190 & 180 & 170 & 160 & 150 & 140 & 130 & 120 & 110 & 100 & 90 & 80 & 70 & 60 & 50 & 40 & 30 & 20 & 10 & 0 & -10 \\ \text { Chemical shift }(\mathrm{ppm})\end{array}$ 


\section{4v ${ }^{1}$ H NMR (500 MHz, Chloroform-d)}

ถุำ

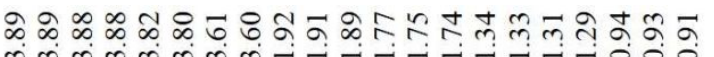

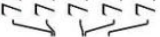

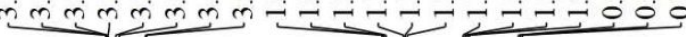

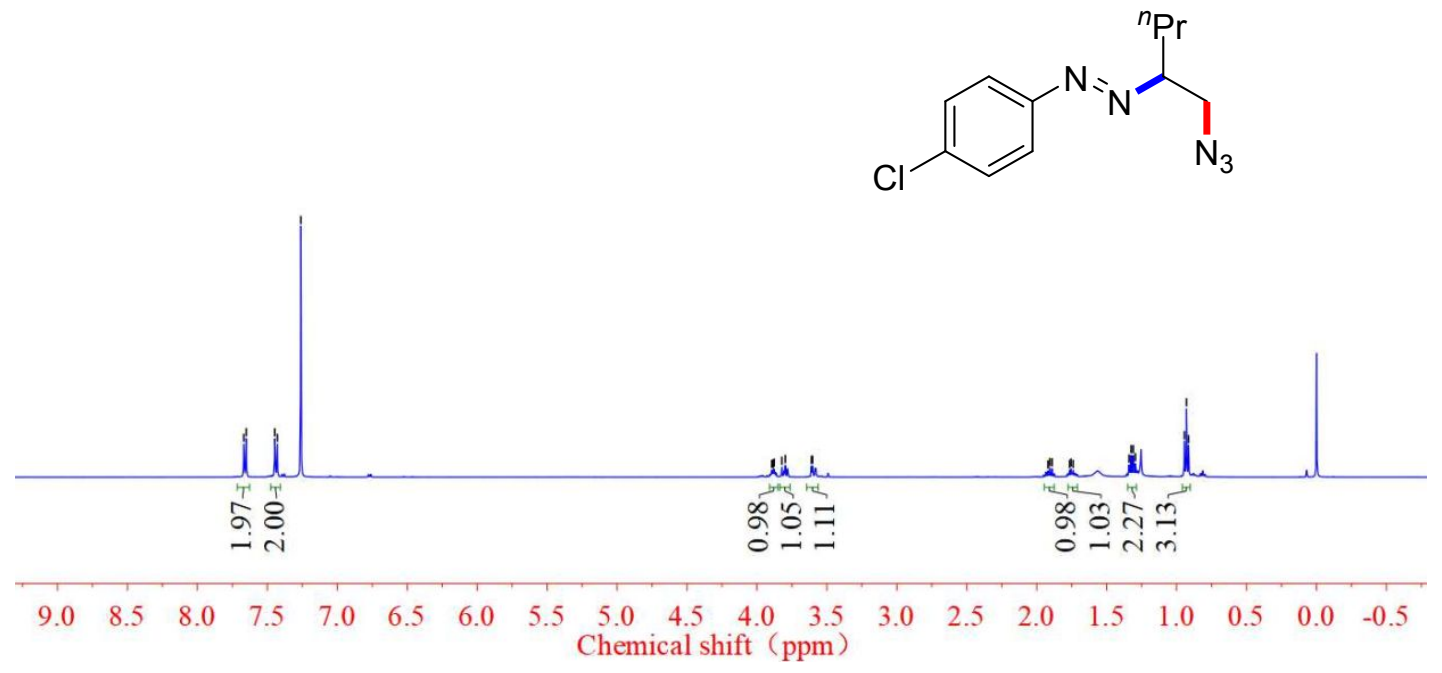

4v ${ }^{13}$ C NMR (126 MHz, Chloroform-d)

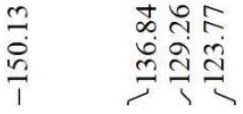

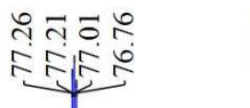

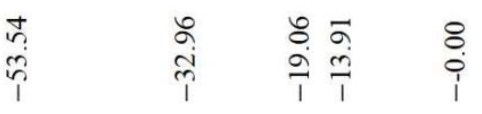

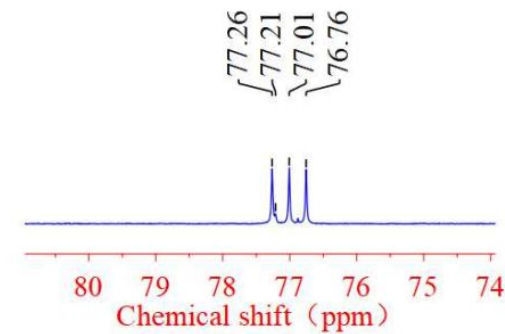<smiles>CC(C)C(CN)N=Nc1ccc(Cl)cc1</smiles>

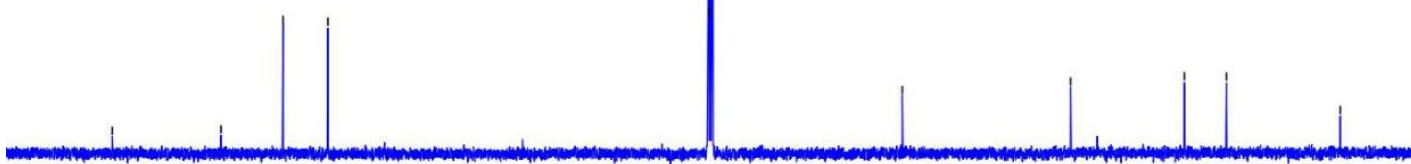

160

$\begin{array}{llllll}150 & 140 & 130 & 120 & 110 & 100\end{array}$

$\begin{array}{ccc}90 & 80 & 70 \\ \text { Chemical shift } & 60\end{array}$ 


\section{4w ${ }^{1}$ H NMR (500 MHz, Chloroform-d)}

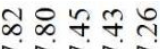

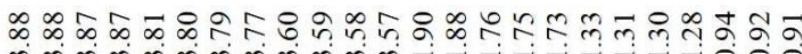
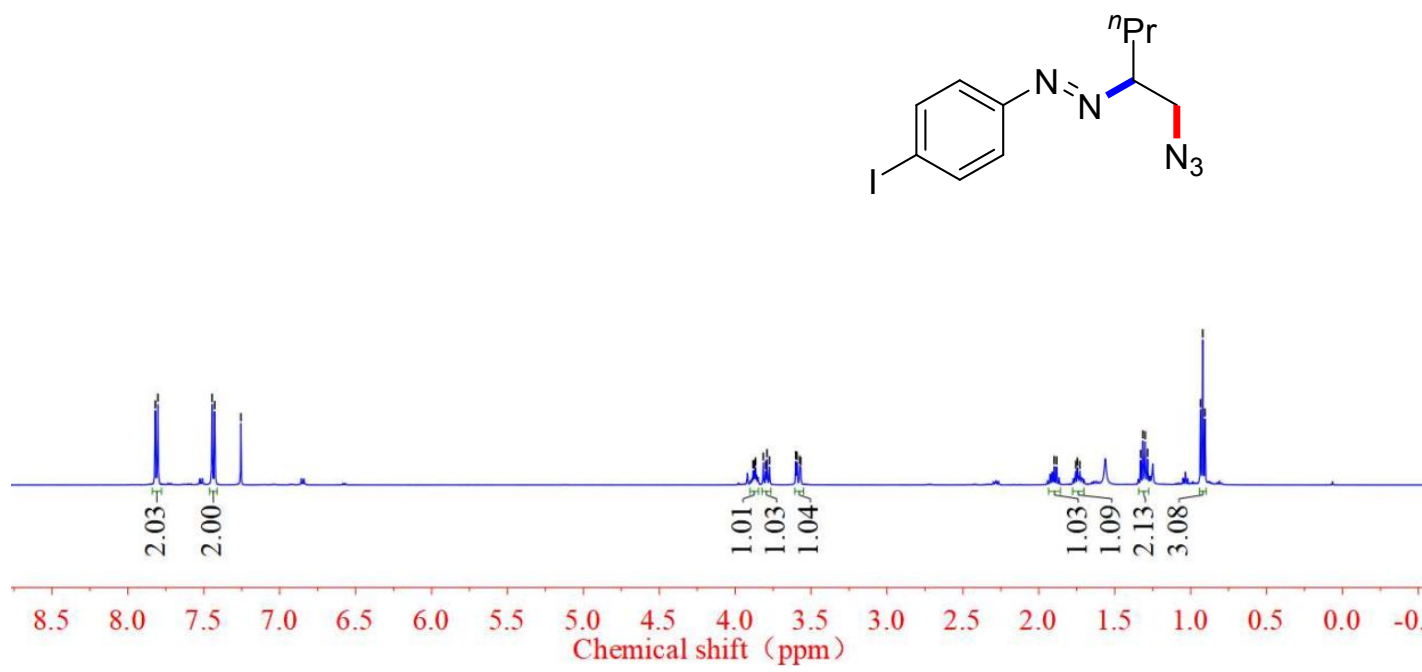

4w ${ }^{13}$ C NMR (126 MHz, Chloroform-d)

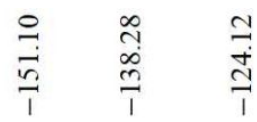

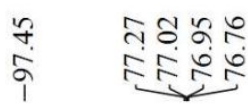

ำ

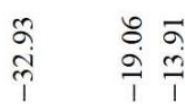

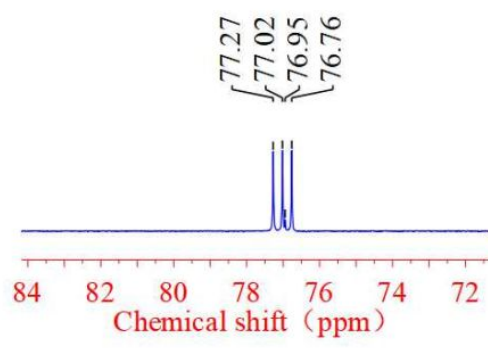<smiles>CCC(CN)N=Nc1ccc(I)cc1</smiles>
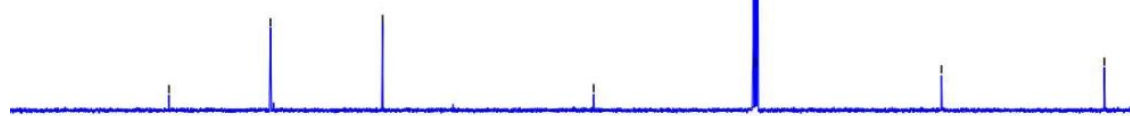

$70 \quad 160$

$\begin{array}{lllll}150 & 140 & 130 & 120\end{array}$

$0 \quad 110 \quad 100$

$\begin{array}{cccc}100 & 90 & 80 & 70 \\ \text { Chemical } & \text { shift } & (\mathrm{ppm})\end{array}$

$\begin{array}{llllll}60 & 50 & 40 & 30 & 20 & 10\end{array}$ 


\section{4x ${ }^{1}$ H NMR (500 MHz, Chloroform-d)}

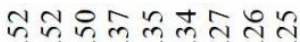

nNañTh

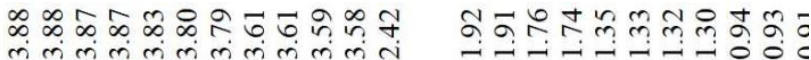

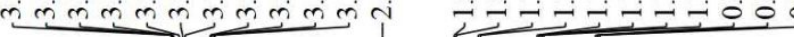

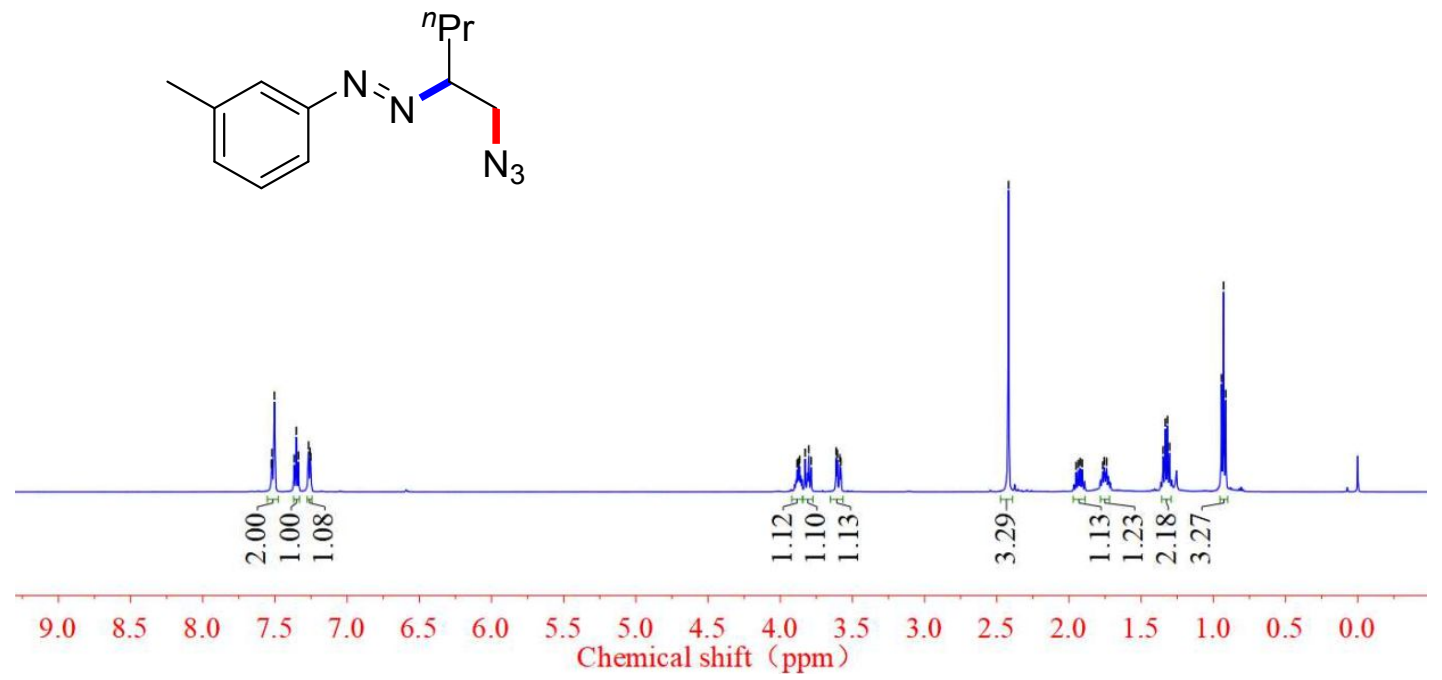

4x ${ }^{13}$ C NMR (126 MHz, Chloroform-d)

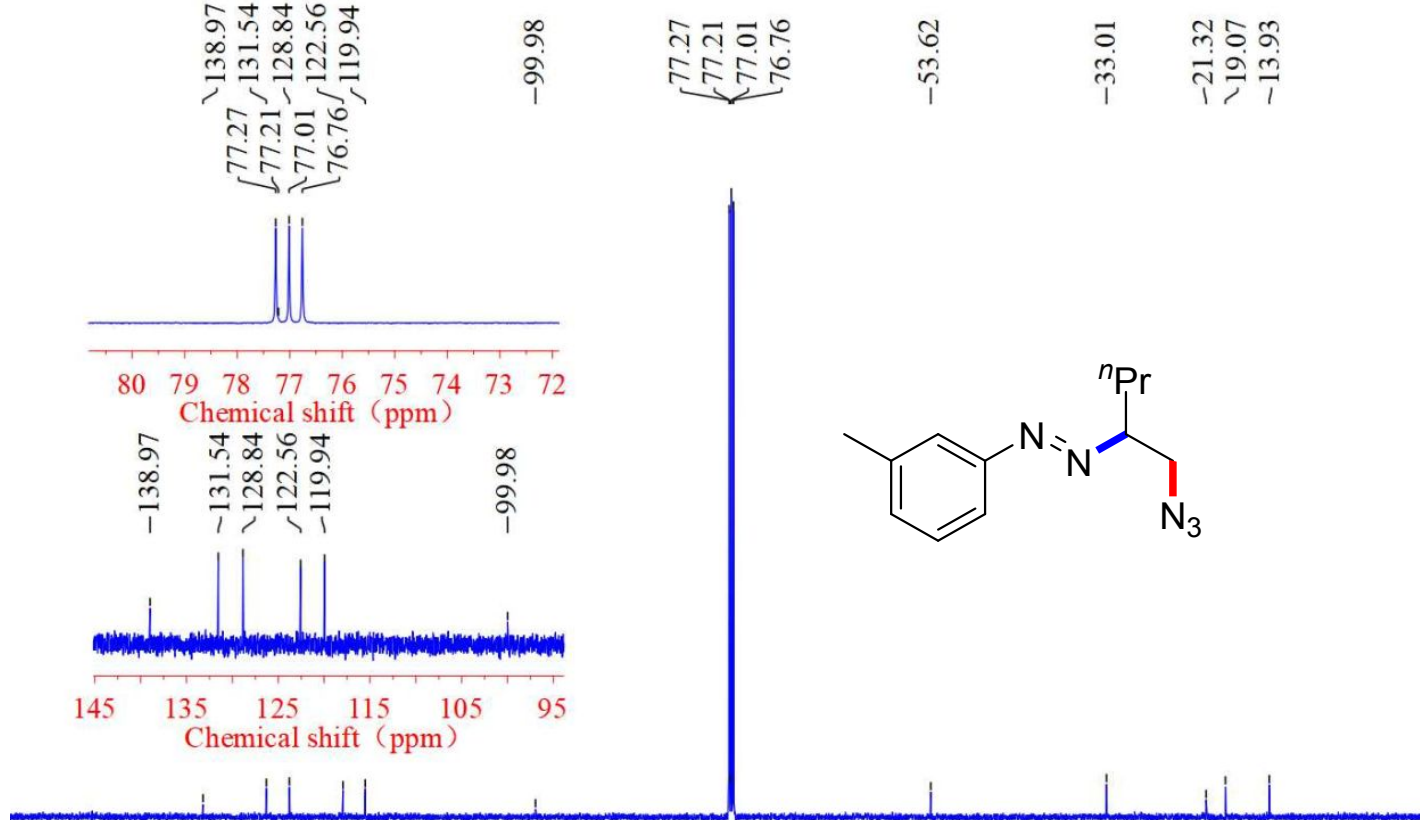

$\begin{array}{llllllllllllllll}60 & 150 & 140 & 130 & 120 & 110 & 100 & \begin{array}{c}90 \\ \text { Chemical shift }\end{array}\left(\begin{array}{c}70 \\ \mathrm{ppm})\end{array}\right. & 60 & 50 & 40 & 30 & 20 & 10 & 0\end{array}$ 


\section{$4 \mathrm{y}^{1} \mathrm{H}$ NMR (500 MHz, Chloroform-d)}

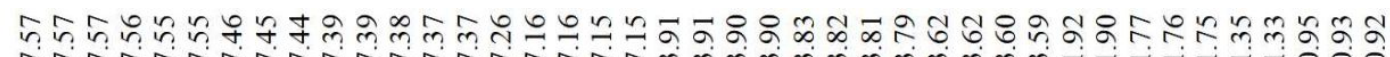

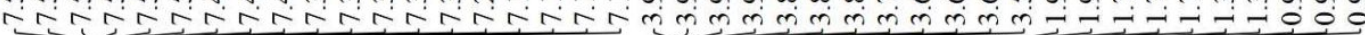<smiles>CC(C)C(C[NH])N=Nc1cccc(F)c1</smiles>

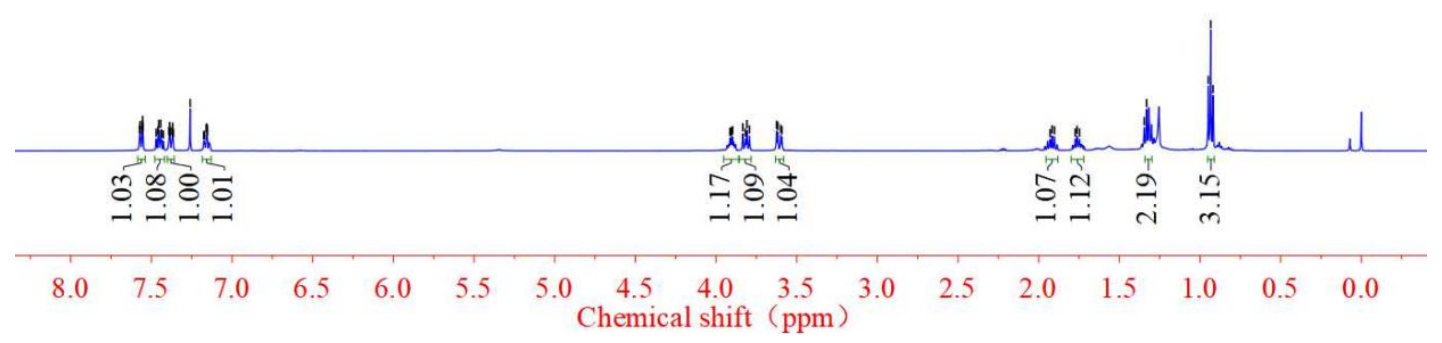

$4 y{ }^{13}$ C NMR (126 MHz, Chloroform-d)

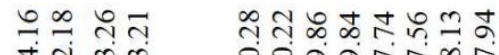

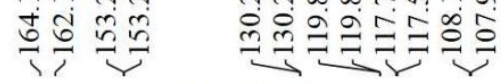

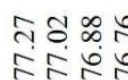

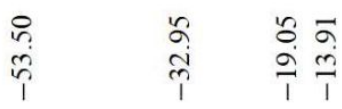

तิ $\widehat{\sigma} \infty$

산ㄷㄴㅇㅠ

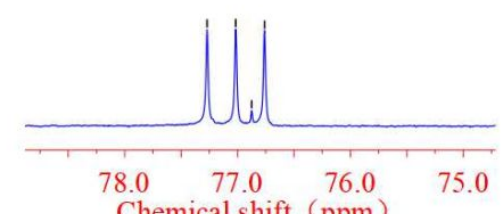

Chemical shift (ppm)

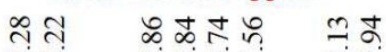

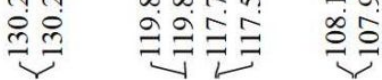<smiles>CC(C)C(CN)N=Nc1cccc(F)c1</smiles>
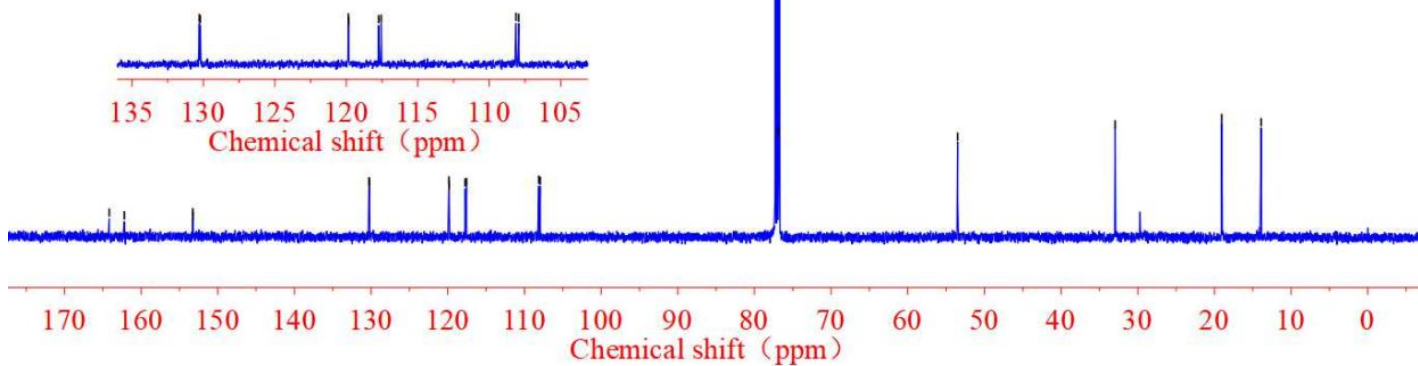
4y ${ }^{19}$ F NMR (471 MHz, Chloroform-d)

$$
\stackrel{+}{\stackrel{\Xi}{\Xi}}
$$<smiles>CC(C)C(CN)N=Nc1cccc(F)c1</smiles>

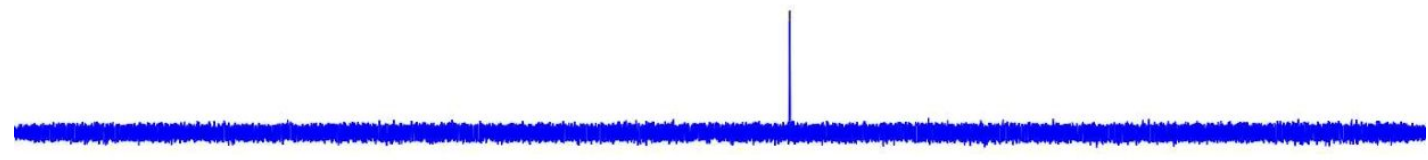

\begin{tabular}{|c|c|c|c|c|c|c|c|c|c|c|c|}
\hline 0 & -20 & -40 & -60 & $\begin{array}{l}-80 \\
\text { Chemi }\end{array}$ & $\begin{array}{c}-100 \\
\text { al shift }\end{array}$ & $\begin{array}{c}-120 \\
(\mathrm{ppm})\end{array}$ & -140 & -160 & -180 & -200 & -22 \\
\hline
\end{tabular}

\section{4z ${ }^{1} \mathrm{H}$ NMR (500 MHz, Chloroform-d)}

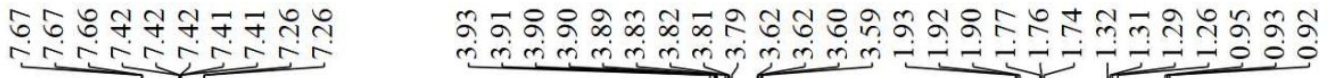

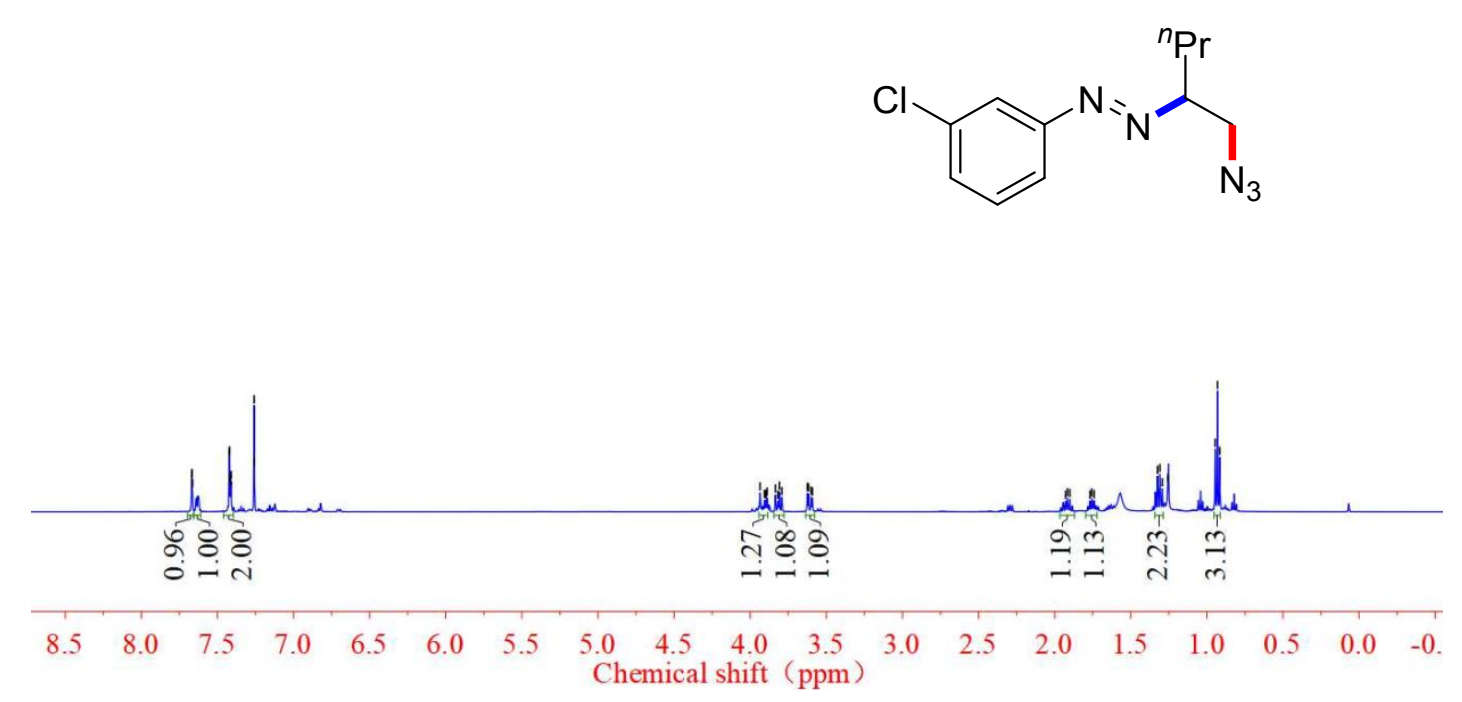




\section{$4 z^{13}$ C NMR (126 MHz, Chloroform-d)}

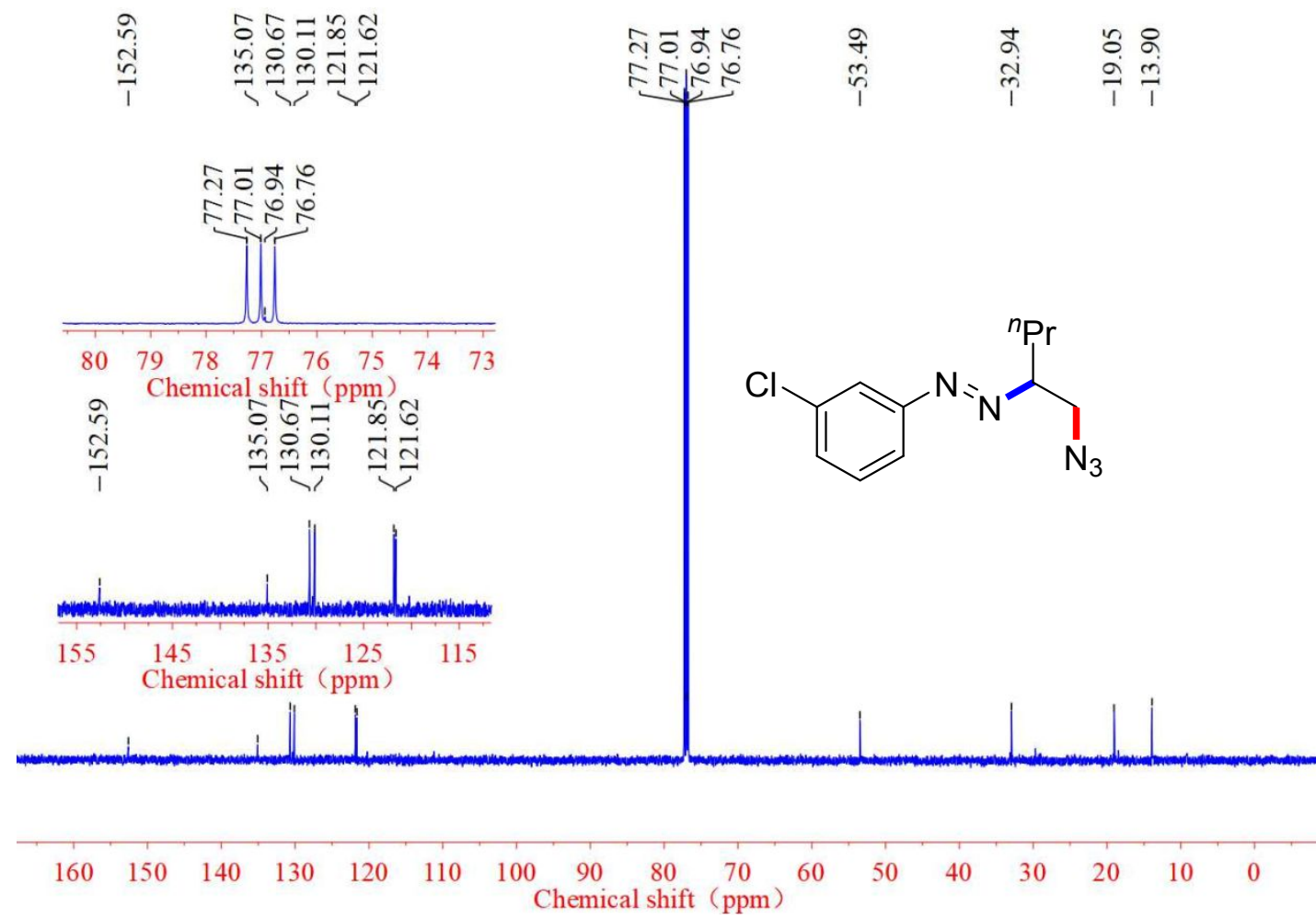

\section{4aa ${ }^{1} \mathrm{H}$ NMR (500 MHz, Chloroform-d)}

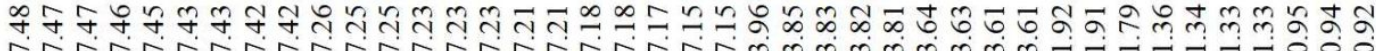

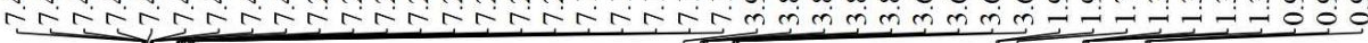

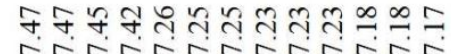
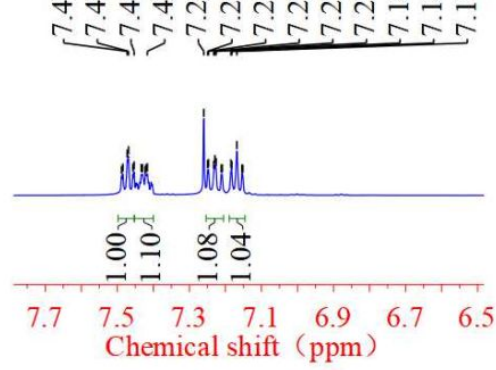<smiles>CC(C)C(CN)N=Nc1ccccc1F</smiles>

$$
\begin{array}{ccccccc}
7.7 & 7.5 & 7.3 & 7.1 & 6.9 & 6.7 & 6.5 \\
\multicolumn{7}{c}{\text { Chemical shift }} \\
(\mathrm{ppm})
\end{array}
$$

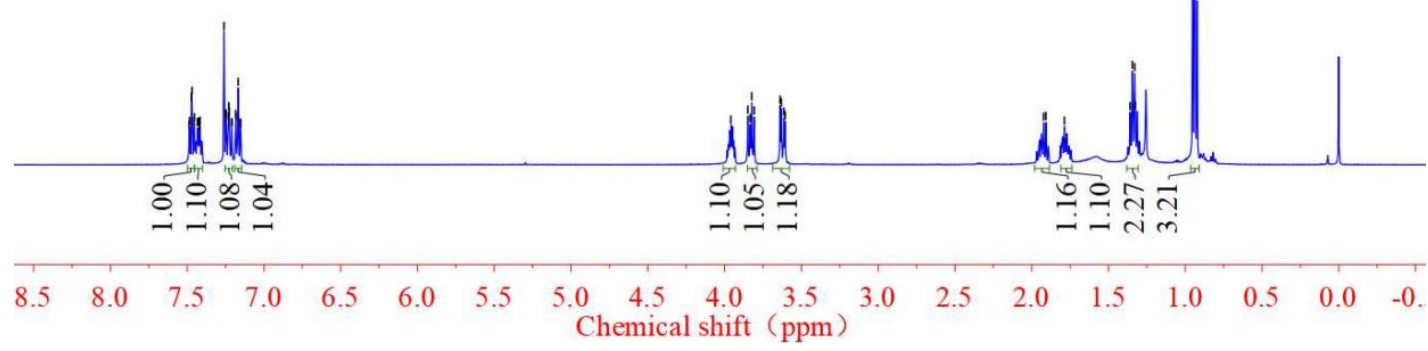




\section{4aa ${ }^{13}$ C NMR (126 MHz, Chloroform- $d$ )}

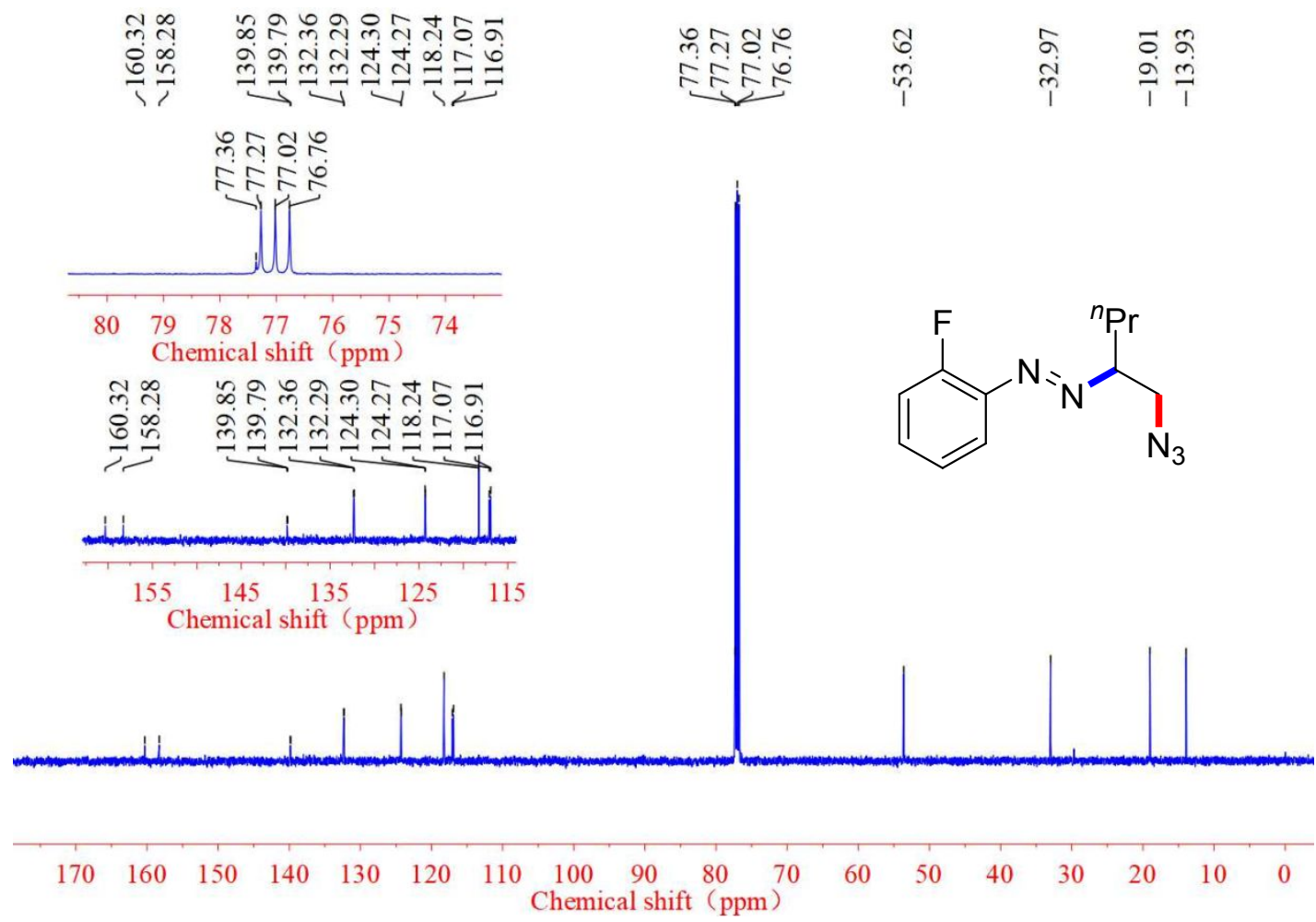

\section{4aa ${ }^{19}$ F NMR (471 MHz, Chloroform-d)}
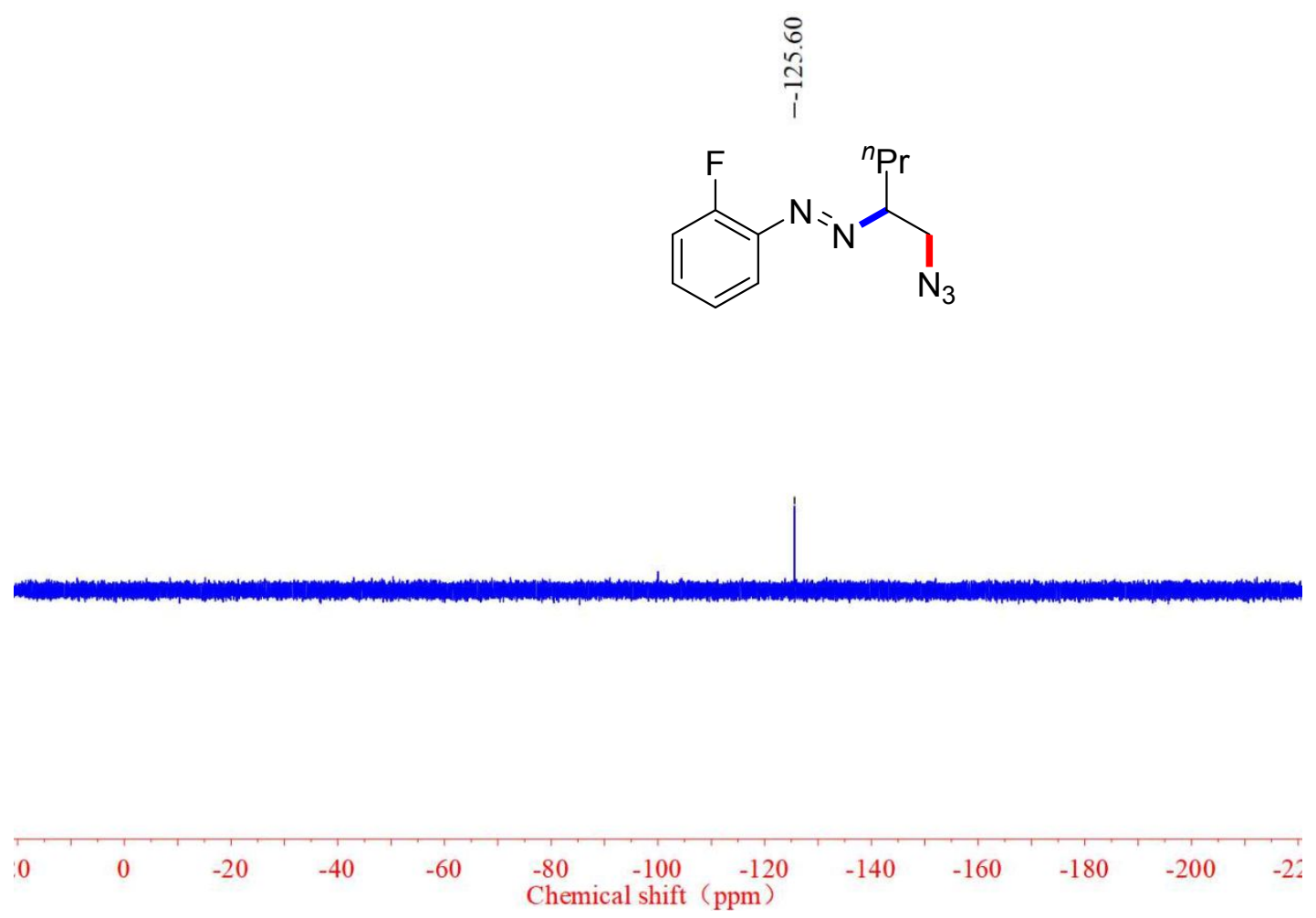


\section{4ab ${ }^{1} \mathrm{H}$ NMR (500 MHz, Chloroform-d)}

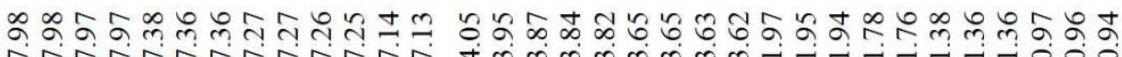

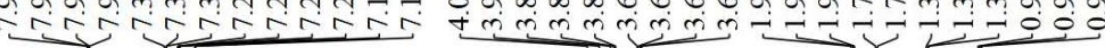<smiles>CCCC(CN)N=Nc1ccccc1I</smiles>

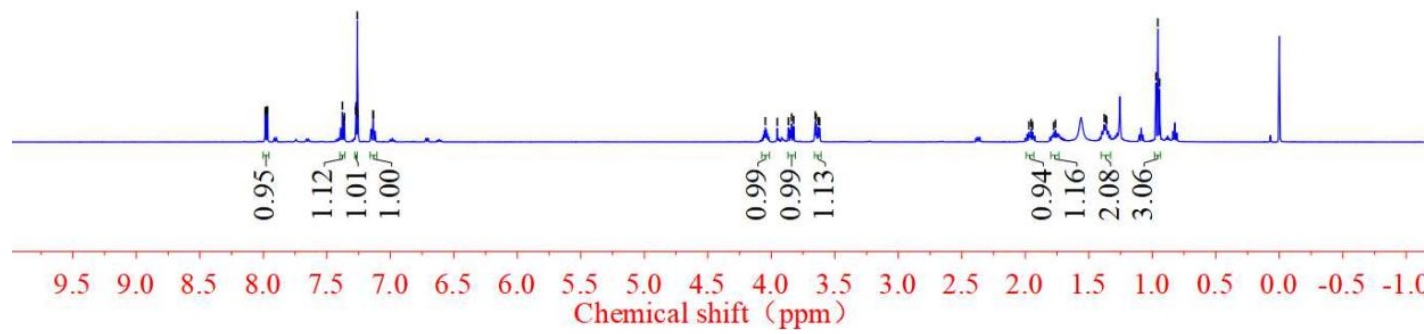

4ab ${ }^{13}$ C NMR (126 MHz, Chloroform-d)

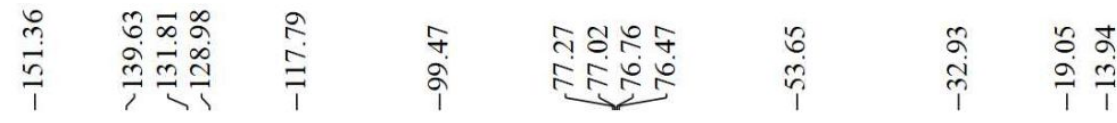
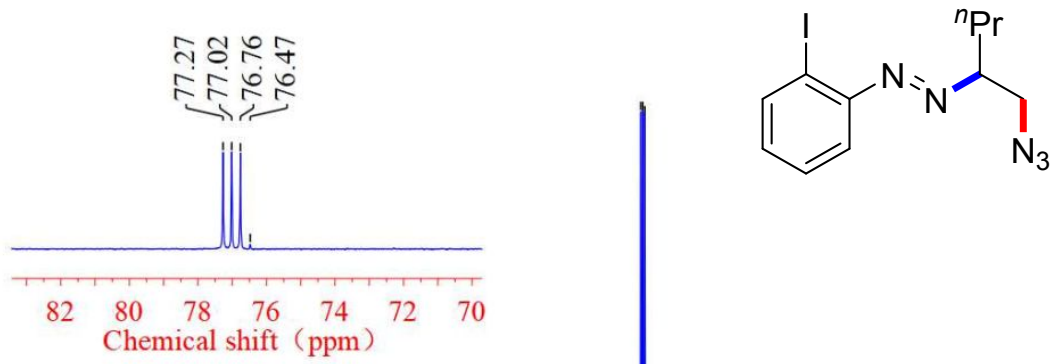

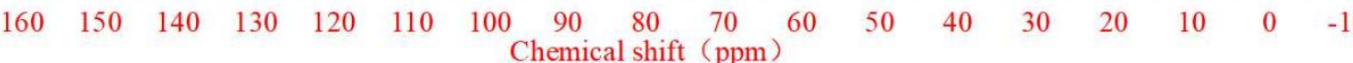




\section{4ac ${ }^{1} \mathrm{H}$ NMR (500 MHz, Chloroform-d)}

के

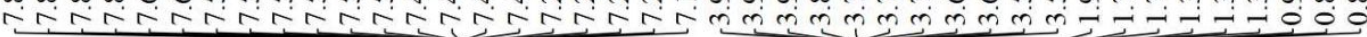<smiles>CCCC(CN)N=Nc1ccccc1[N+](=O)[O-]</smiles>

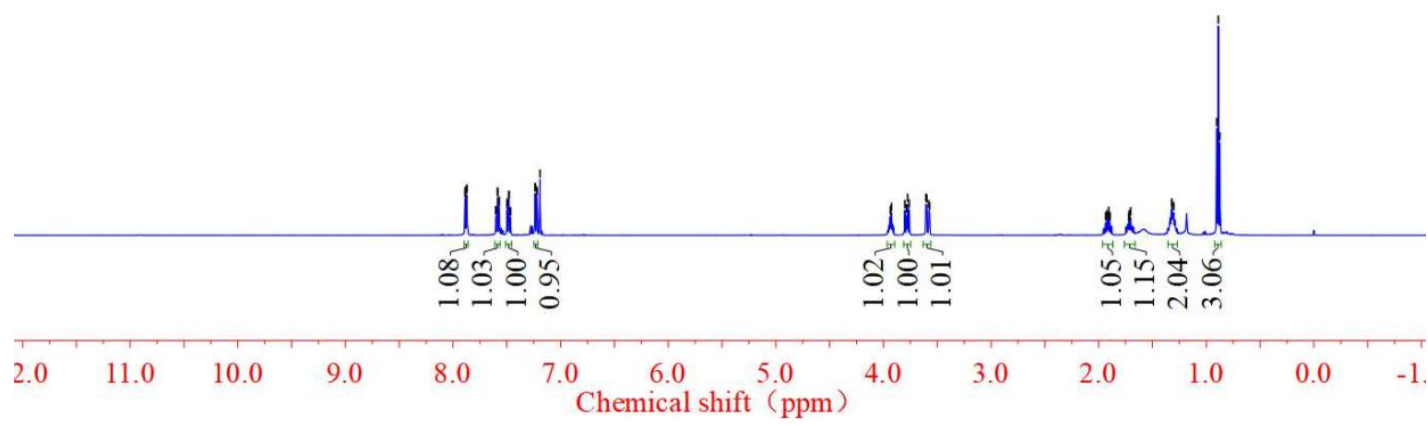

4ac ${ }^{13} \mathrm{C}$ NMR (126 MHz, Chloroform-d)

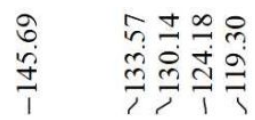

भे ते ठำ

다듀

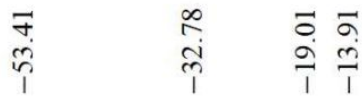
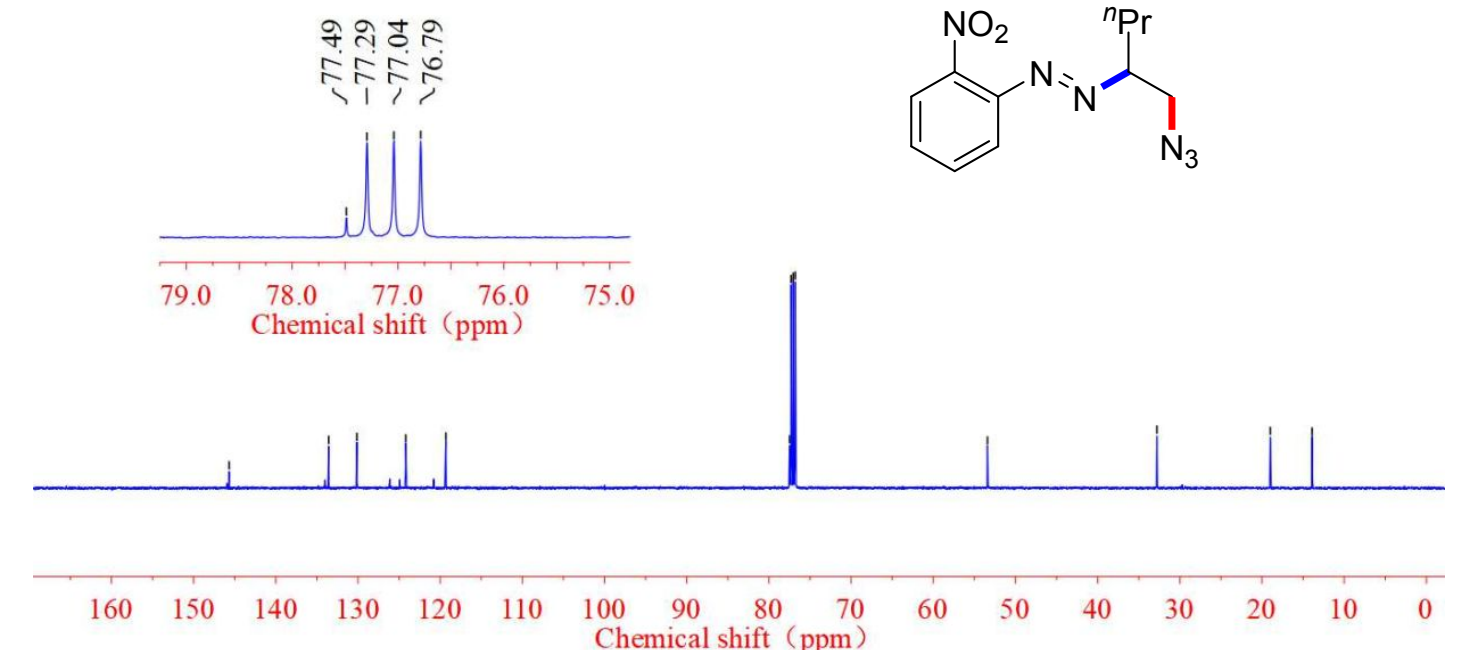


\section{4ad ${ }^{1} \mathrm{H}$ NMR (500 MHz, Chloroform-d)}

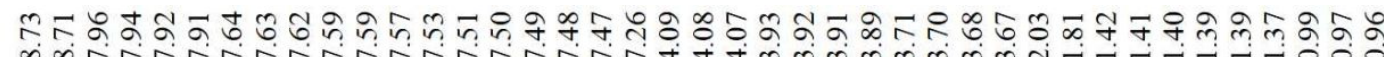

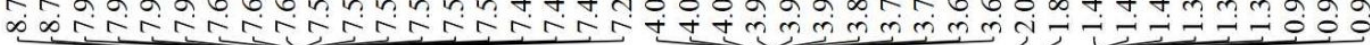<smiles>CC(C)C(CN)N=Nc1cccc2ccccc12</smiles>

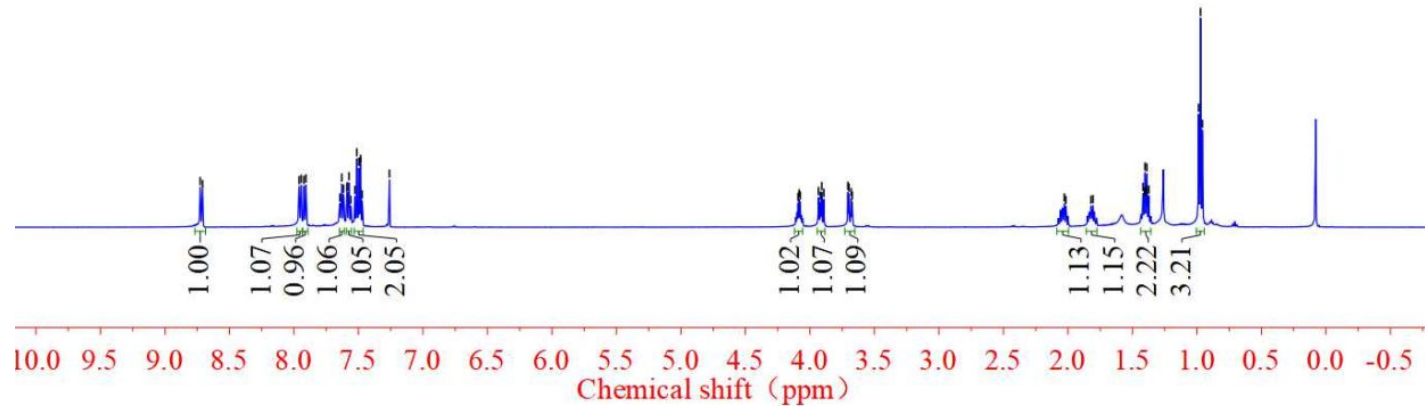

4ad ${ }^{13} \mathrm{C}$ NMR (126 MHz, Chloroform-d)

请:

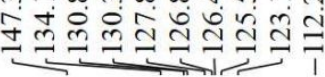

iุะ ำ

हर्शे के के के के
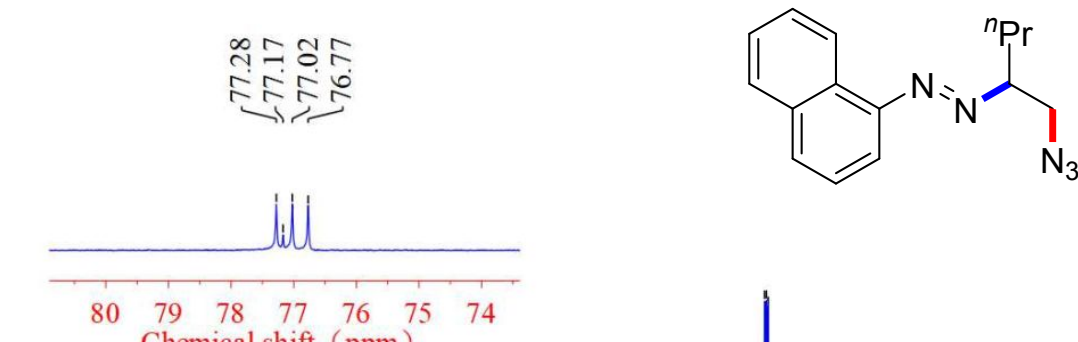

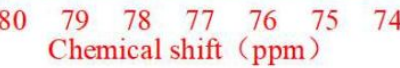

$\begin{array}{llllllllllllllllllll}180 & 170 & 160 & 150 & 140 & 130 & 120 & 110 & \begin{array}{c}100 \\ \text { Chemical shift }\end{array}\left(\begin{array}{c}70 \\ (\mathrm{ppm})\end{array}\right. & 60 & 50 & 40 & 30 & 20 & 10 & 0 & -10\end{array}$ 


\section{4ae ${ }^{1} \mathrm{H}$ NMR (500 MHz, Chloroform-d)}

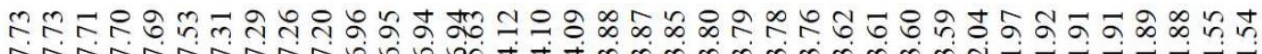

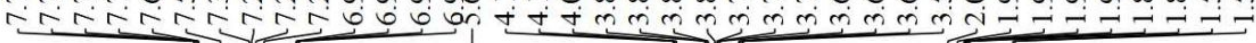<smiles>COc1ccc(NNC(CN)CCCCOc2cc(=O)oc3ccccc23)cc1</smiles>

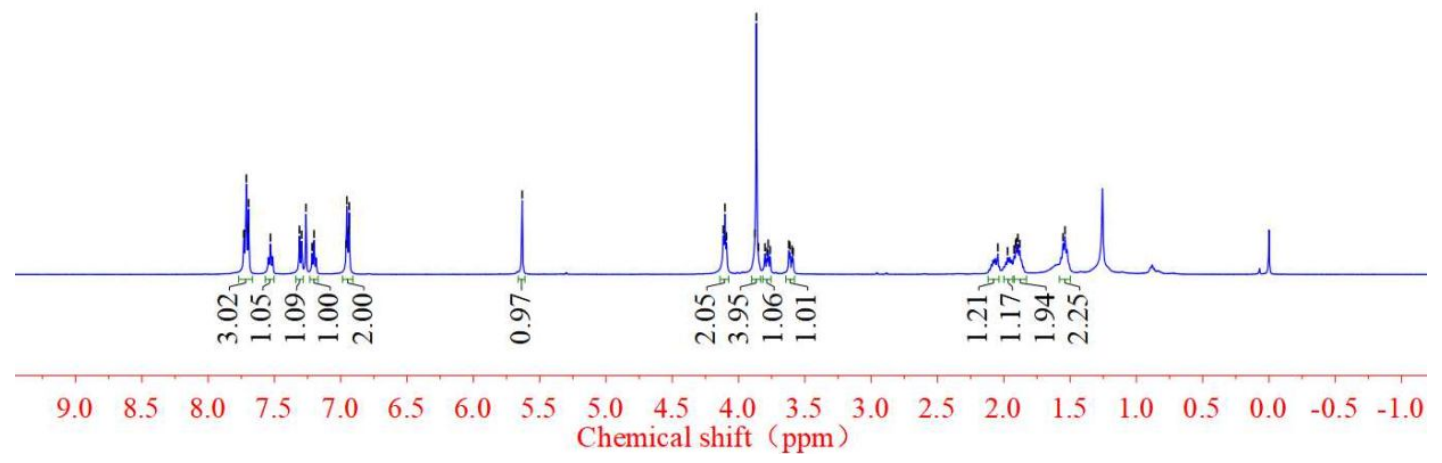

\section{4ae ${ }^{13}$ C NMR (126 MHz, Chloroform-d)}

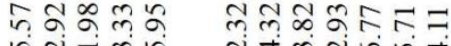

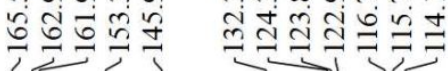

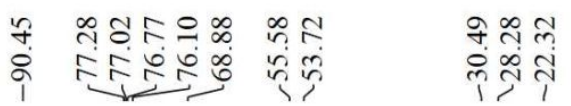
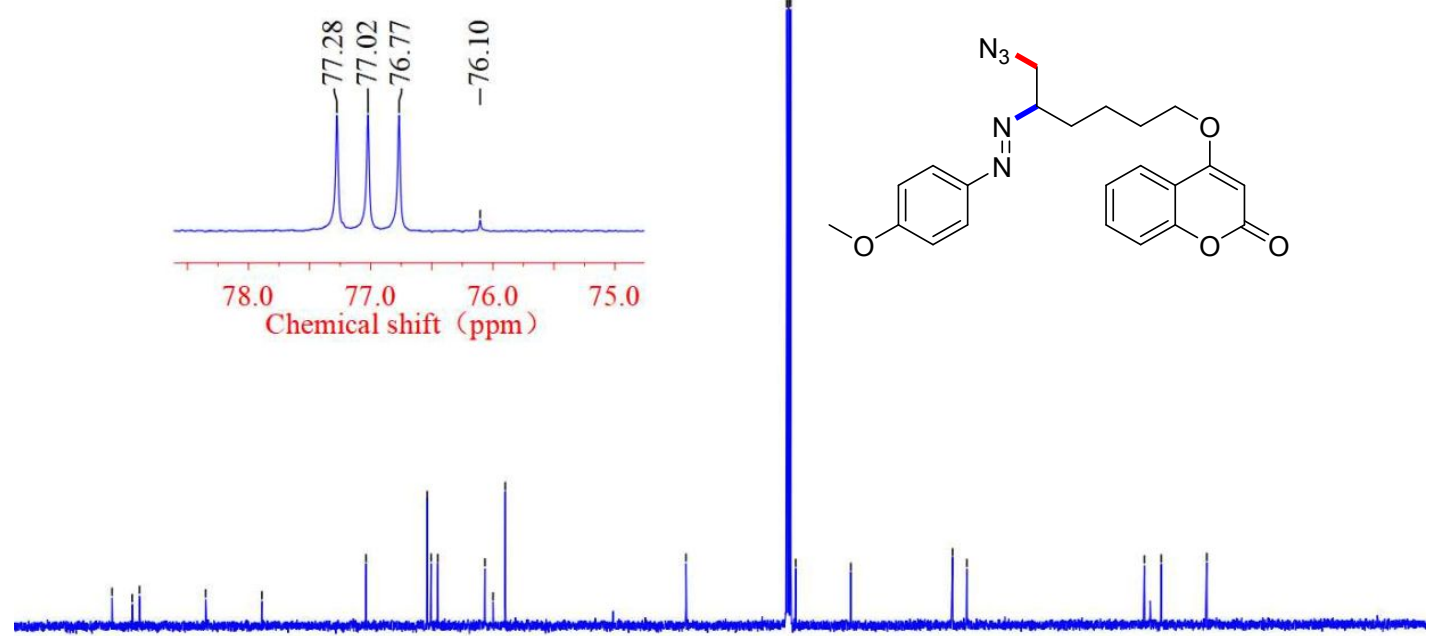

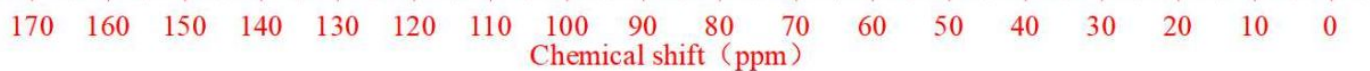




\section{4af ${ }^{1} \mathrm{H}$ NMR (500 MHz, Chloroform-d)}

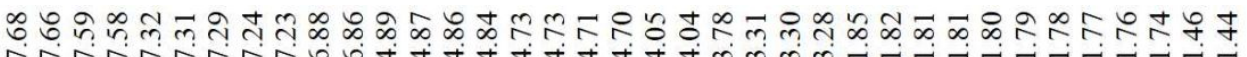

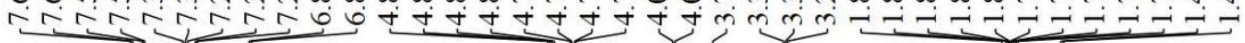

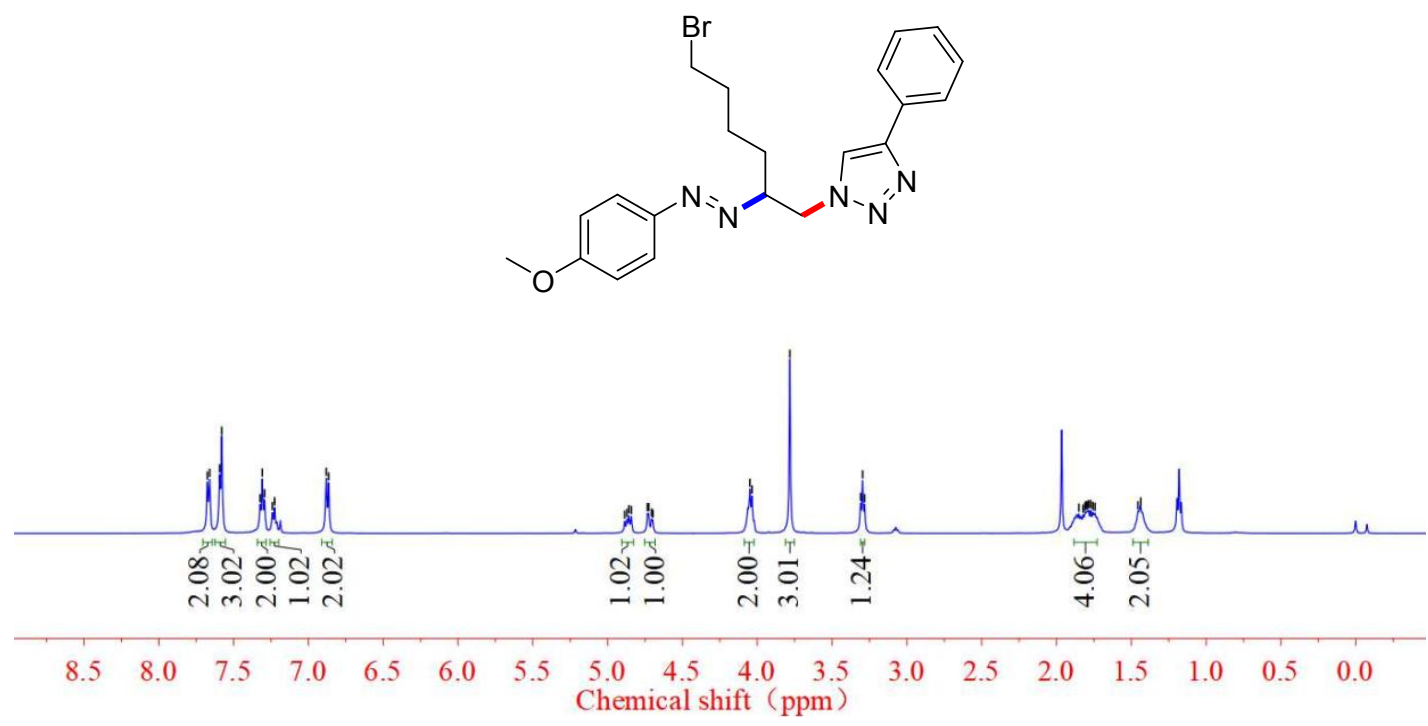

4af ${ }^{13}$ C NMR (126 MHz, Chloroform-d)

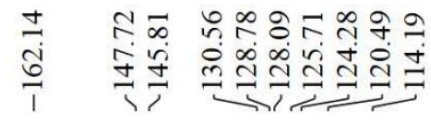

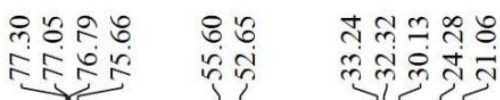
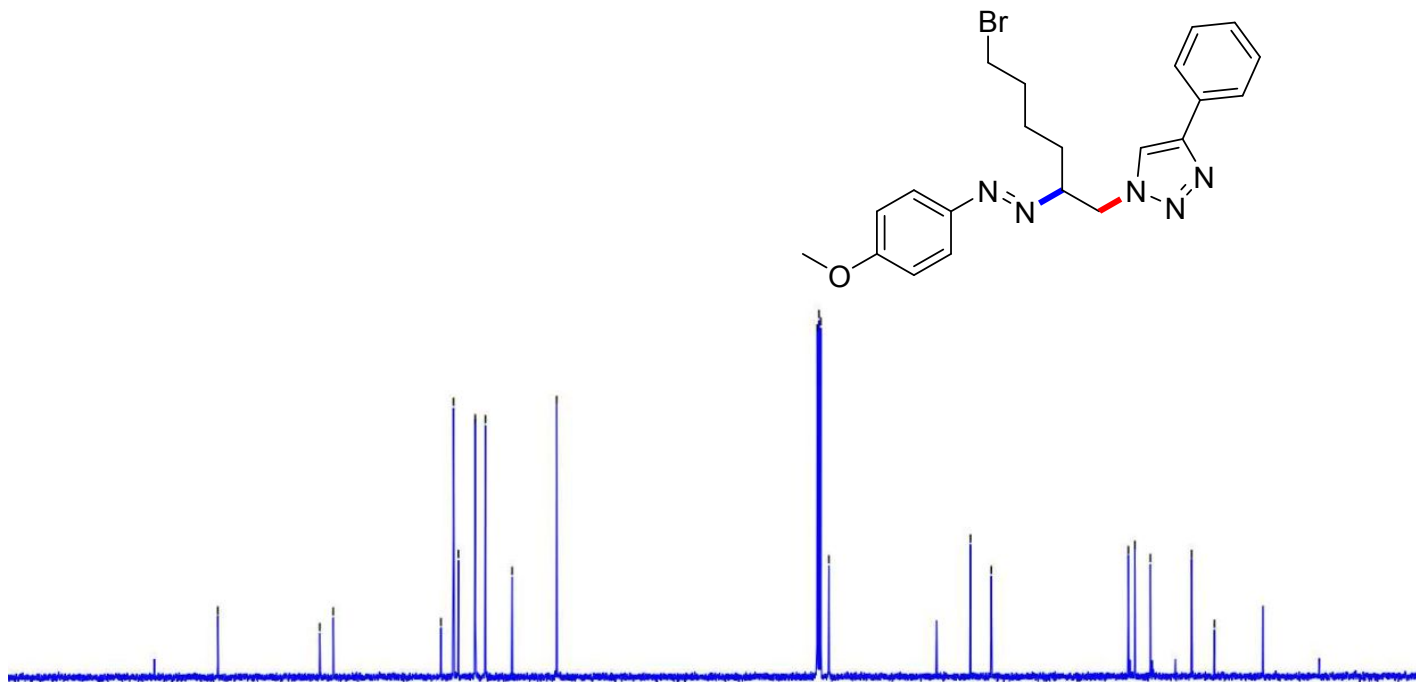

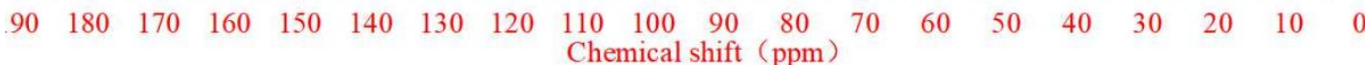




\section{4ag ${ }^{1}$ H NMR (500 MHz, Chloroform-d)}

œ نतल

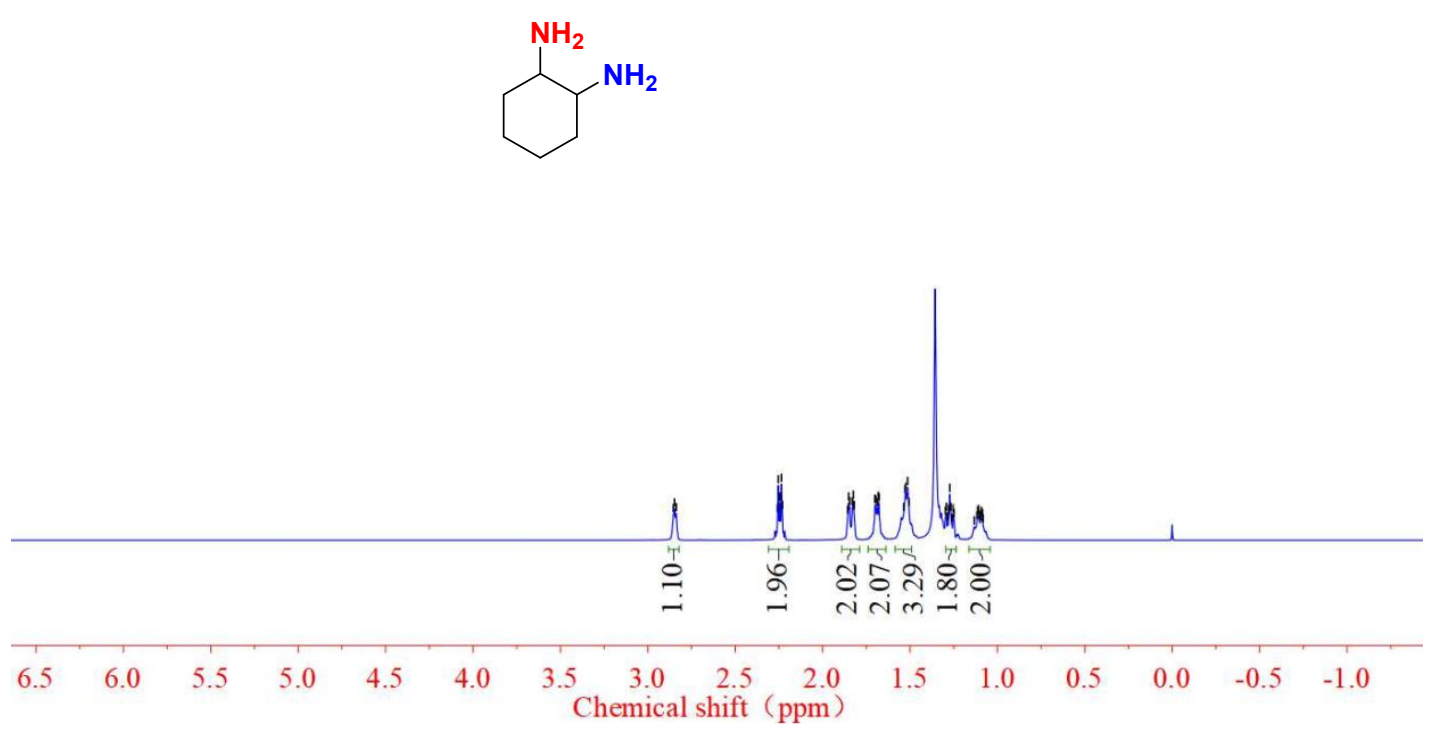

\section{4ah ${ }^{1} \mathrm{H}$ NMR (500 MHz, Chloroform-d)}

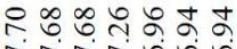

inition

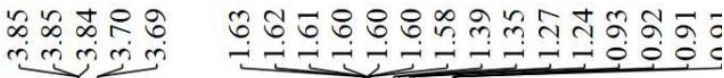
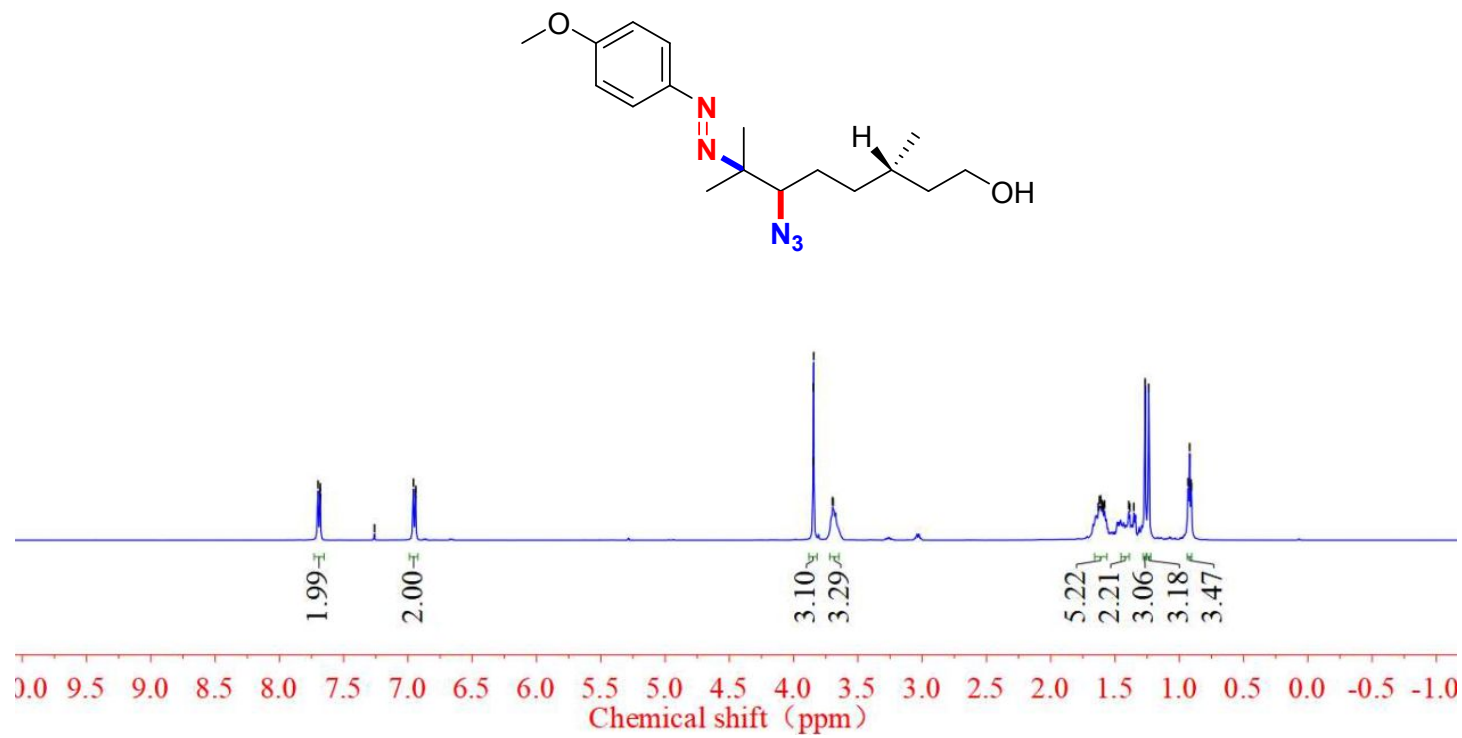


\section{4ah ${ }^{13}$ C NMR (126 MHz, Chloroform-d)}

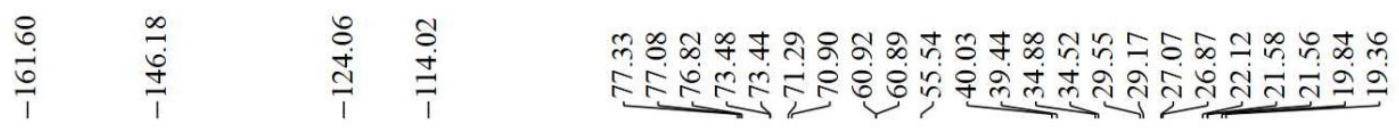
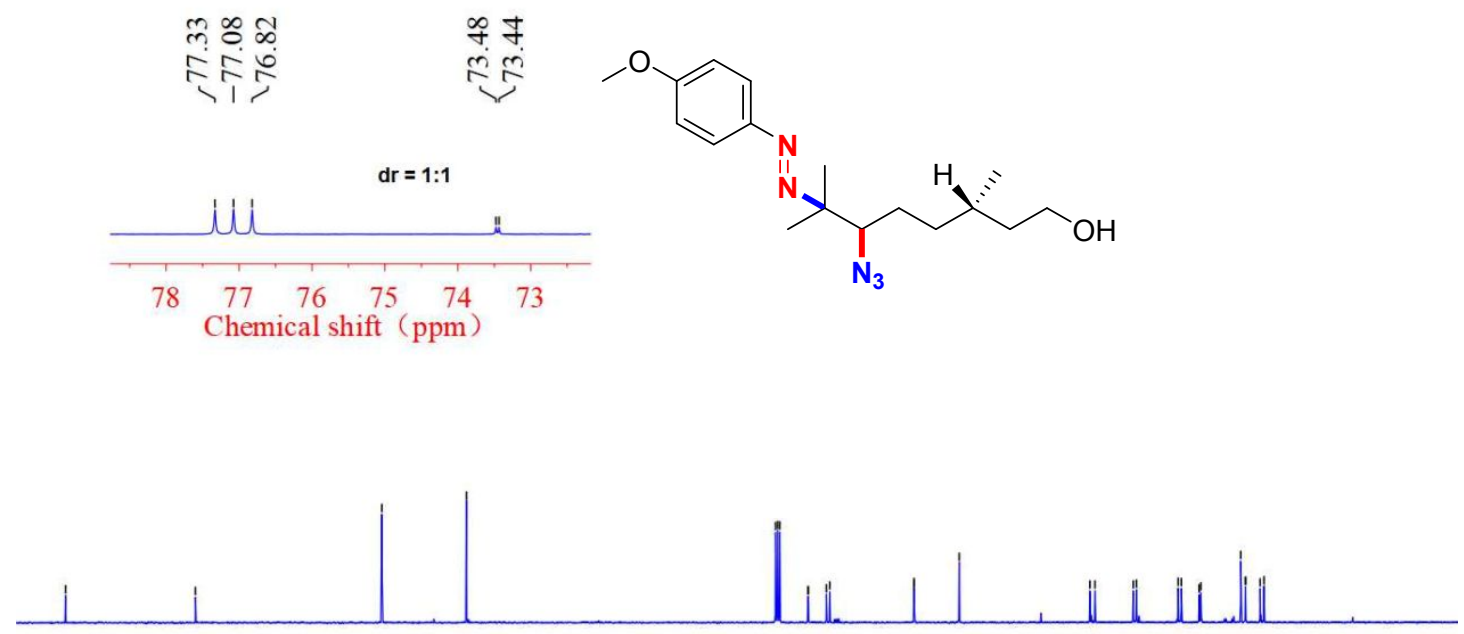

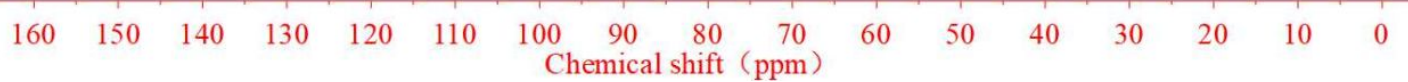

\section{4ai ${ }^{1} \mathrm{H}$ NMR (500 MHz, Chloroform-d)}

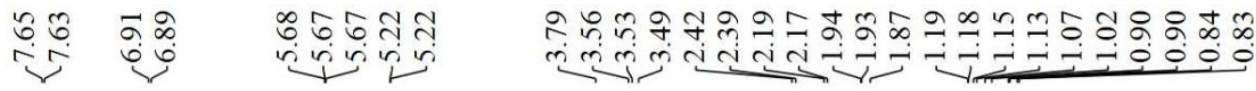
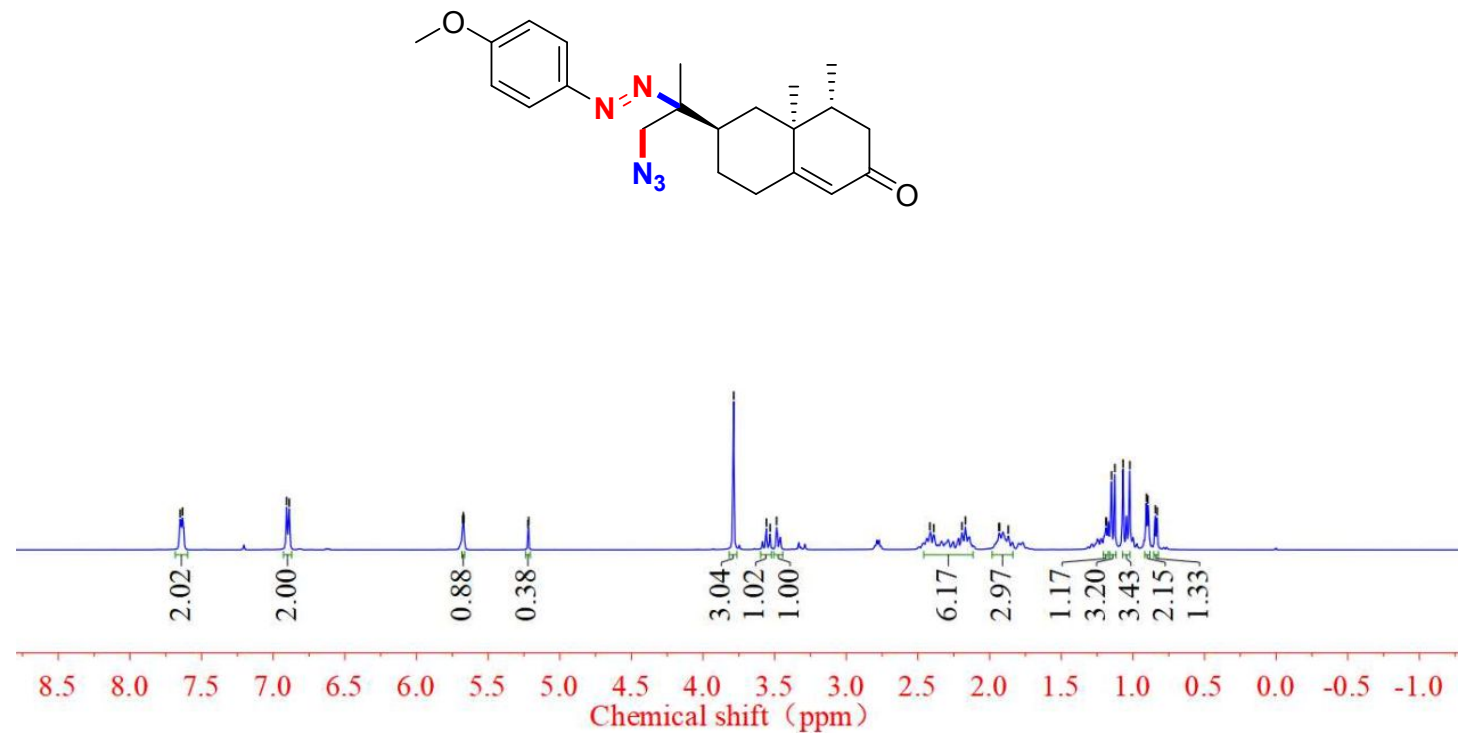


\section{4ai ${ }^{13} \mathrm{C}$ NMR (126 MHz, Chloroform-d)}

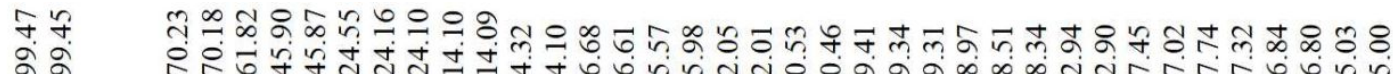

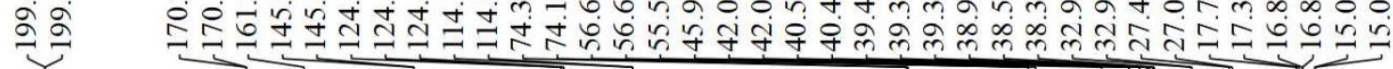
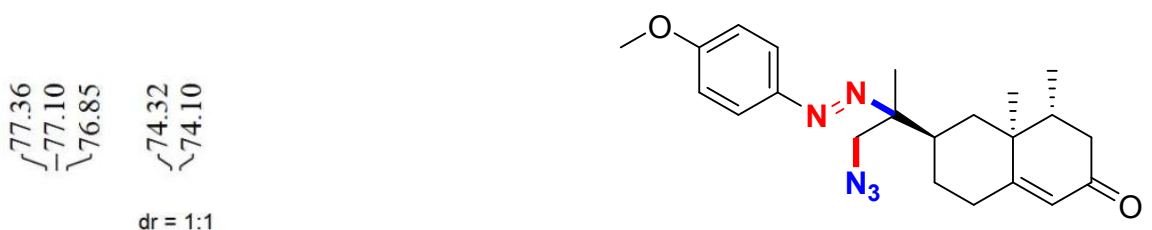

iii

818079787776757473727170

Chemical shift (ppm)

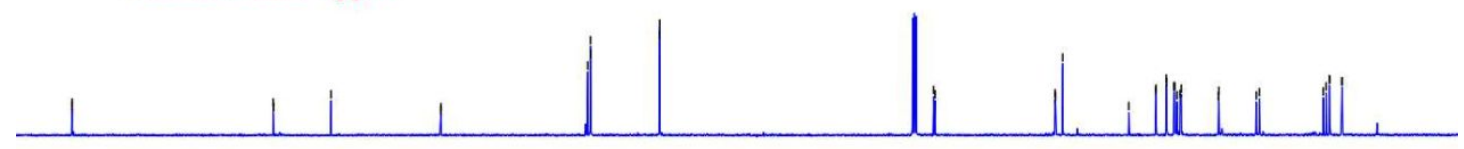

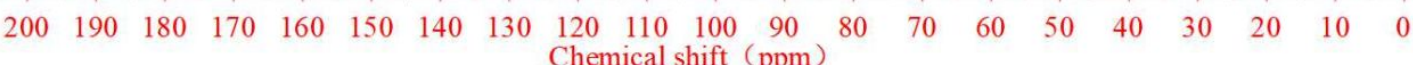

\section{4ai ${ }^{1} \mathrm{H}$ NMR (500 MHz, DMSO- $\left.d_{6}\right)$}

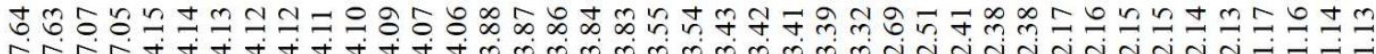

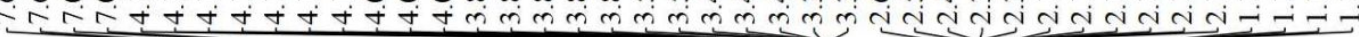<smiles>CCOC(=O)C1(C(=O)OCC)CC(CN)C(CN=Nc2ccc(OC)cc2)C1</smiles>

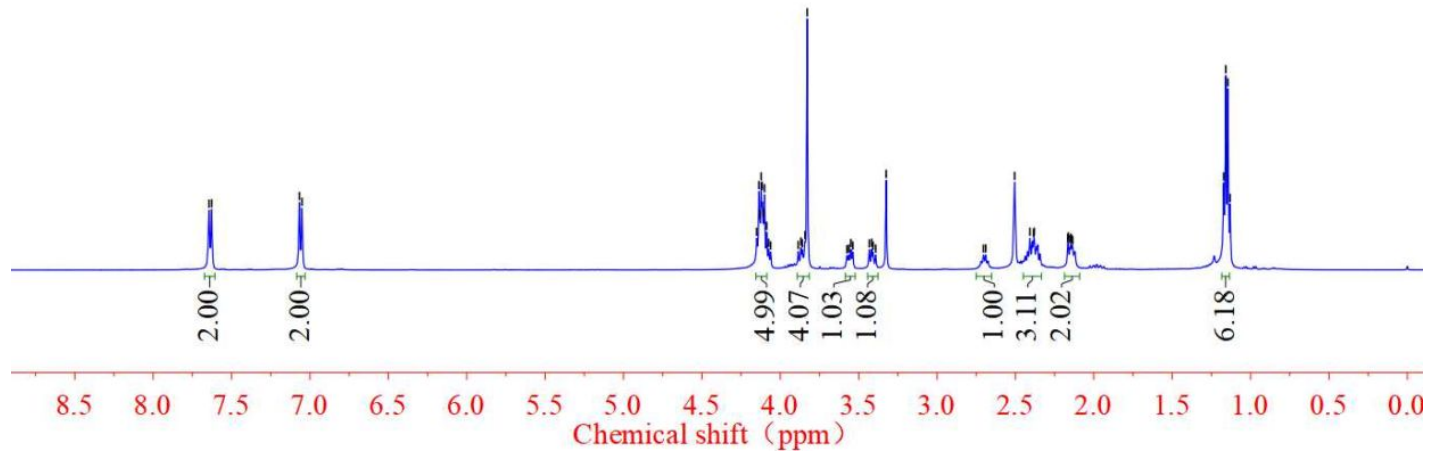


4ai ${ }^{13} \mathrm{C}$ NMR (126 MHz, DMSO- $d_{6}$ )

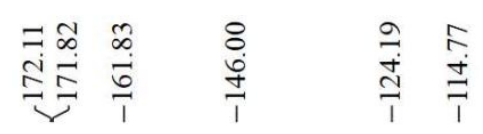

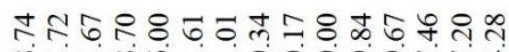

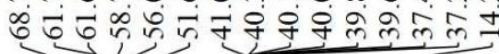<smiles>CCOC(=O)C1(C(=O)OCC)CC(CN)C(CN=Nc2ccc(OC)cc2)C1</smiles>

$\begin{array}{llllllllllllllllll}180 & 170 & 160 & 150 & 140 & 130 & 120 & 110 \begin{array}{c}100 \\ \text { Chemical shift }(\mathrm{ppm})\end{array} & \begin{array}{c}80 \\ 70\end{array} & 60 & 50 & 40 & 30 & 20 & 10 & 0 & -1(\mathrm{c})\end{array}$

\section{High Resolution Mass Spectrometry}<smiles>CCCC(CN)NNc1ccc(OC)cc1</smiles>

4a

HRMS (ESI): $[\mathrm{M}+\mathrm{H}]^{+}$Calculated for $\mathrm{C}_{12} \mathrm{H}_{17} \mathrm{~N}_{5} \mathrm{OH}$ : 248.1506, Found 248.1506.

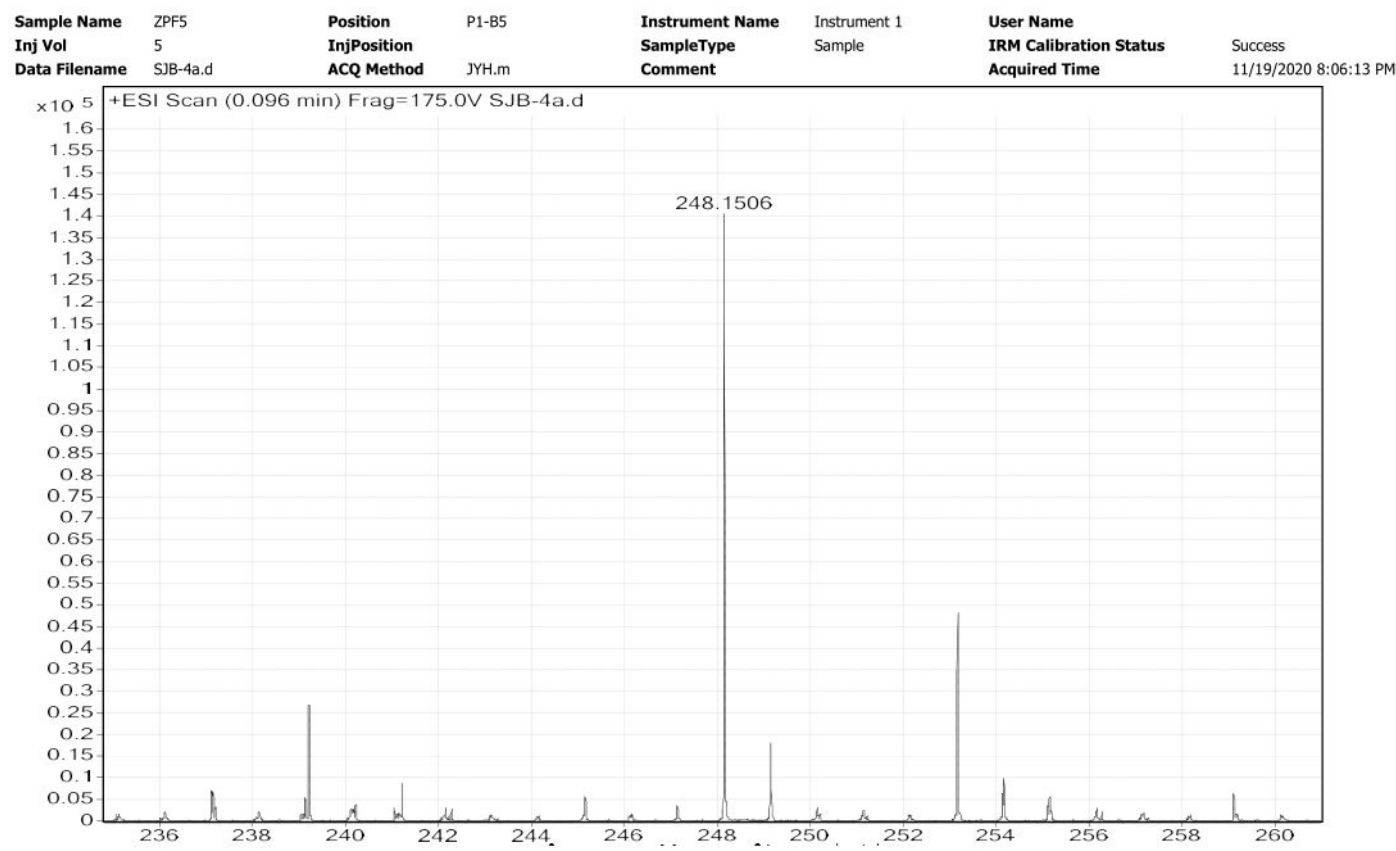




\begin{tabular}{|c|r|r|r|r|r|r|r|}
\hline Formula (M) & Score (MFG) & Mass & Mass (MFG) & \multicolumn{1}{c|}{$\mathrm{m} / \mathrm{z}$ (Calc) } & Diff (ppm) & DBE & $\mathrm{m} / \mathrm{z}$ \\
\hline $\mathrm{C} 12 \mathrm{H} 17 \mathrm{N50}$ & 100 & 247.1433 & 247.1433 & 248.1506 & -0.05 & & 248.1506 \\
\hline
\end{tabular}

4b

HRMS (ESI): $[\mathrm{M}+\mathrm{H}]^{+}$Calculated for $\mathrm{C}_{13} \mathrm{H}_{19} \mathrm{~N}_{5} \mathrm{OH}: 262.1663$, Found 262.1662 .

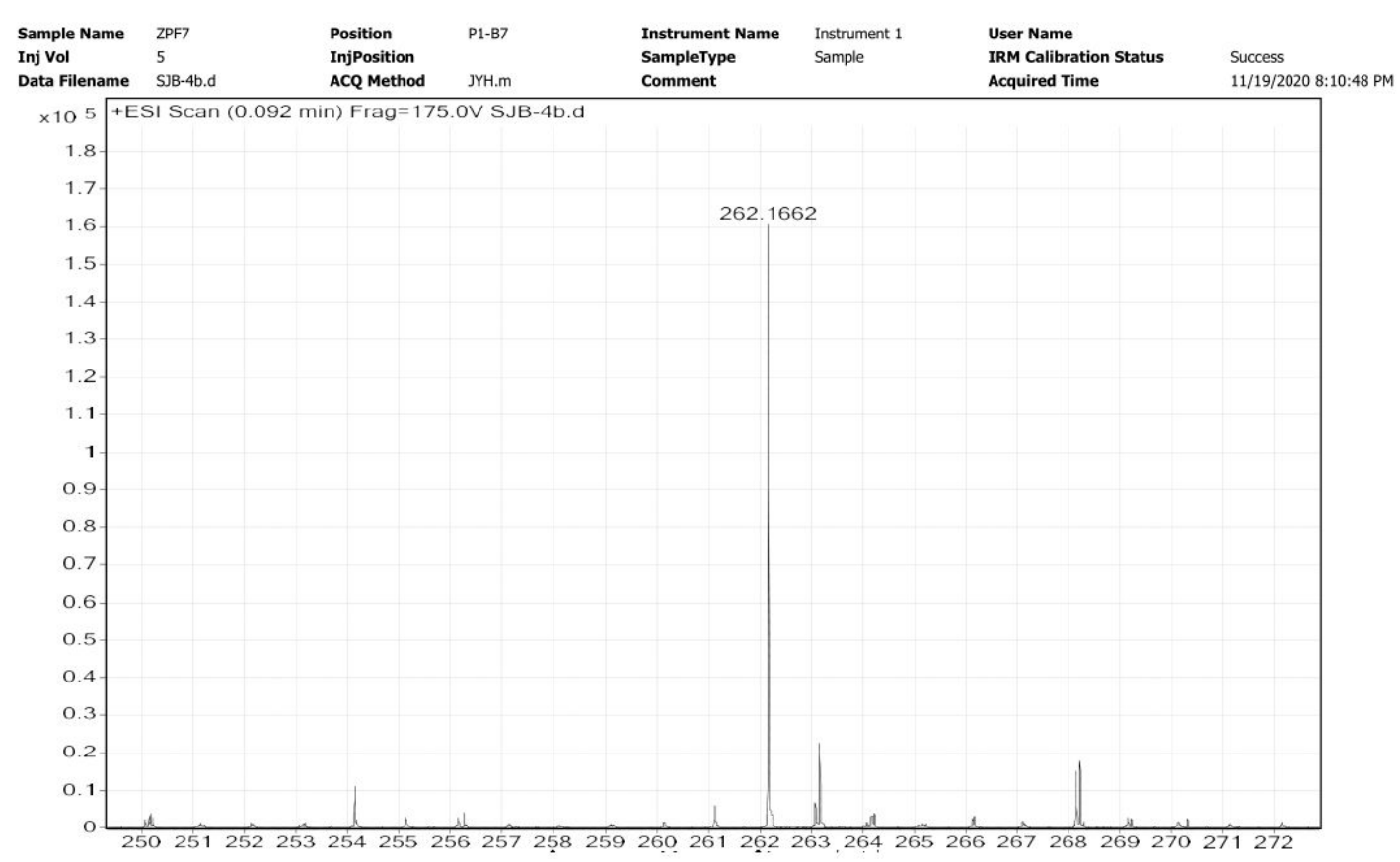

\begin{tabular}{|c|r|r|r|r|r|r|r|}
\hline Formula (M) & Score (MFG) & Mass & Mass (MFG) & $\mathrm{m} / \mathrm{z}$ (Calc) & Diff (ppm) & DBE & $\mathrm{m} / \mathrm{z}$ \\
\hline C13 H19 N5 O & 99.99 & 261.1589 & 261.159 & 262.1662 & 0.14 & & 7 \\
\hline
\end{tabular}<smiles>COc1ccc(/N=N/C(C)(C)C(C)N)cc1</smiles>

$4 d$

HRMS (ESI): $[\mathrm{M}+\mathrm{Na}]^{+}$Calculated for $\mathrm{C}_{12} \mathrm{H}_{17} \mathrm{~N}_{5} \mathrm{ONa}$ : 270.1325, Found 270.1325 . 


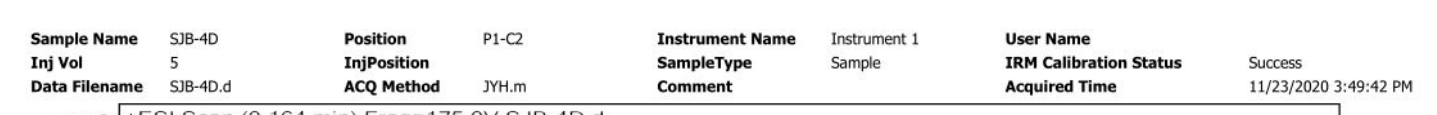

Data Filename SJB-4D.d ACQ Method JYH.m
$\times 1 0 6 \longdiv { + E S I ~ S c a n ~ ( 0 . 1 6 4 ~ m i n ) ~ F r a g = 1 7 5 . 0 V ~ S J B - 4 D . d ~ }$ Comment Acquired Time 11/23/2020 3:49:42 PM

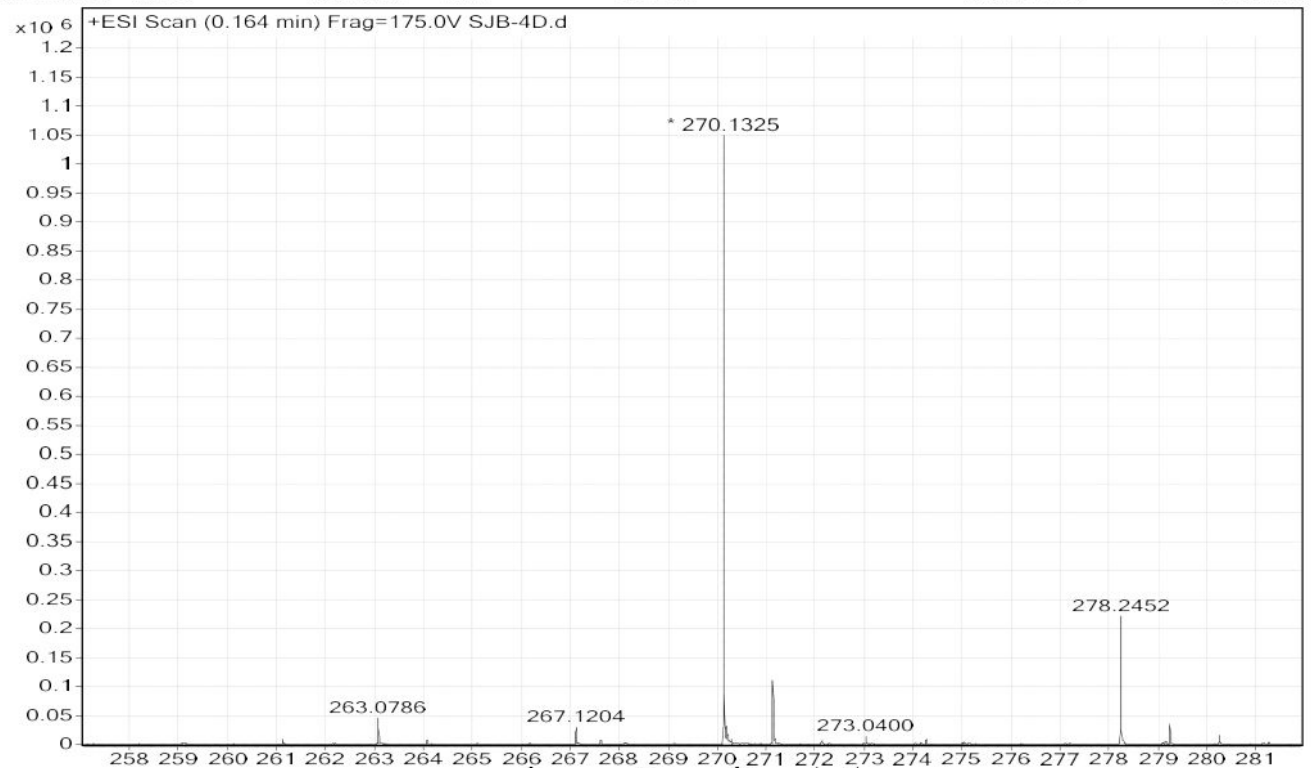

\begin{tabular}{|c|r|r|r|r|r|r|r|}
\hline Formula (M) & Score (MFG) & Mass & Mass (MFG) & m/z (Calc) & Diff (ppm) & DBE & $\mathrm{m} / \mathrm{z}$ \\
\hline C12 H17 N5 O & 100 & 247.1433 & 247.1433 & 270.1325 & 0.13 & 7 & 270.1325 \\
\hline
\end{tabular}<smiles>COc1ccc(N=NC2CCCCC2N)cc1</smiles>

$4 e$

HRMS (ESI): $[\mathrm{M}+\mathrm{Na}]^{+}$Calculated for $\mathrm{C}_{13} \mathrm{H}_{17} \mathrm{~N}_{5} \mathrm{ONa}$ : 282.1325, Found 282.1328.

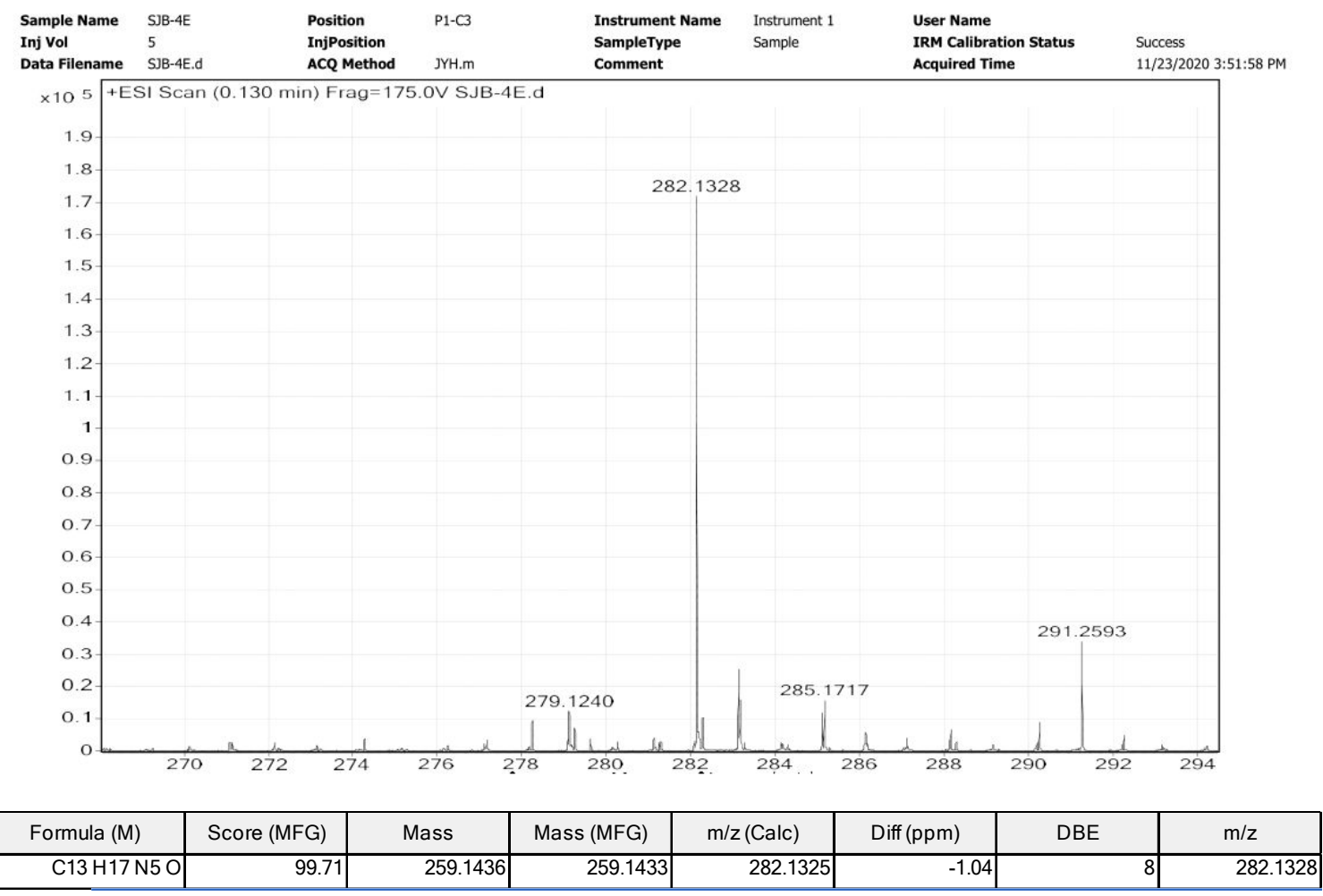




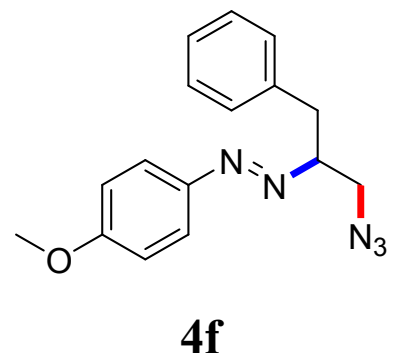

HRMS (ESI): $[\mathrm{M}+\mathrm{Na}]^{+}$Calculated for $\mathrm{C}_{16} \mathrm{H}_{17} \mathrm{~N}_{5} \mathrm{ONa}$ : 318.1325, Found 318.1328 .

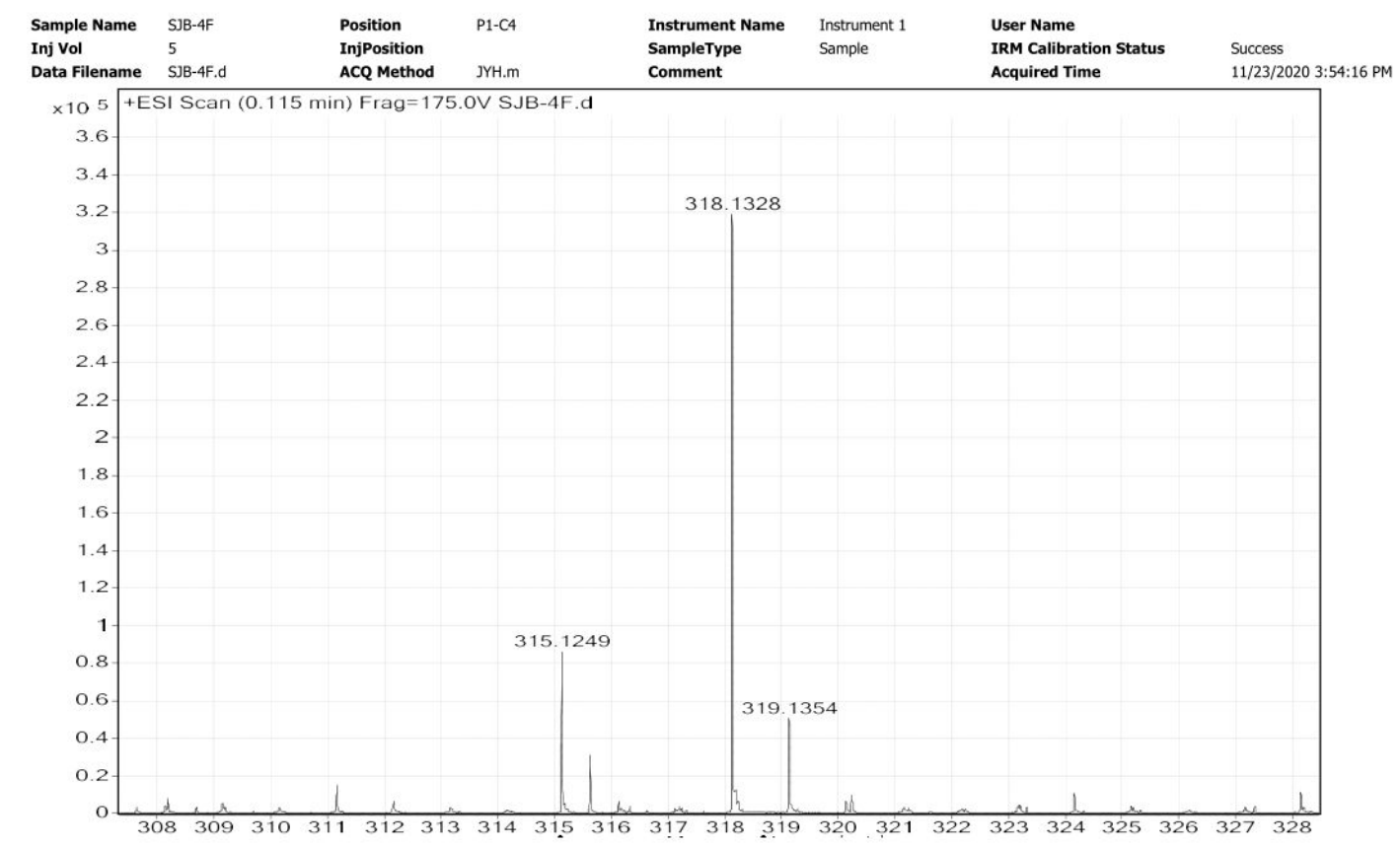

\begin{tabular}{|c|c|c|c|c|c|c|c|}
\hline Formula (M) & Score (MFG) & Mass & Mass (MFG) & $\mathrm{m} / \mathrm{z}$ (Calc) & Diff (ppm) & DBE & $\mathrm{m} / \mathrm{z}$ \\
\hline $\mathrm{C} 16 \mathrm{H} 17 \mathrm{~N} 5 \mathrm{O}$ & 99.74 & 295.1436 & 295.1433 & 318.1325 & -0.91 & 11 & 318.1328 \\
\hline
\end{tabular}<smiles>COc1ccc(N=NC(CN)CO)cc1</smiles>

$4 \mathrm{~h}$

HRMS (ESI): $[\mathrm{M}+\mathrm{Na}]^{+}$Calculated for $\mathrm{C}_{13} \mathrm{H}_{19} \mathrm{~N}_{5} \mathrm{O}_{2} \mathrm{Na}: 300.1431$, Found 300.1429. 


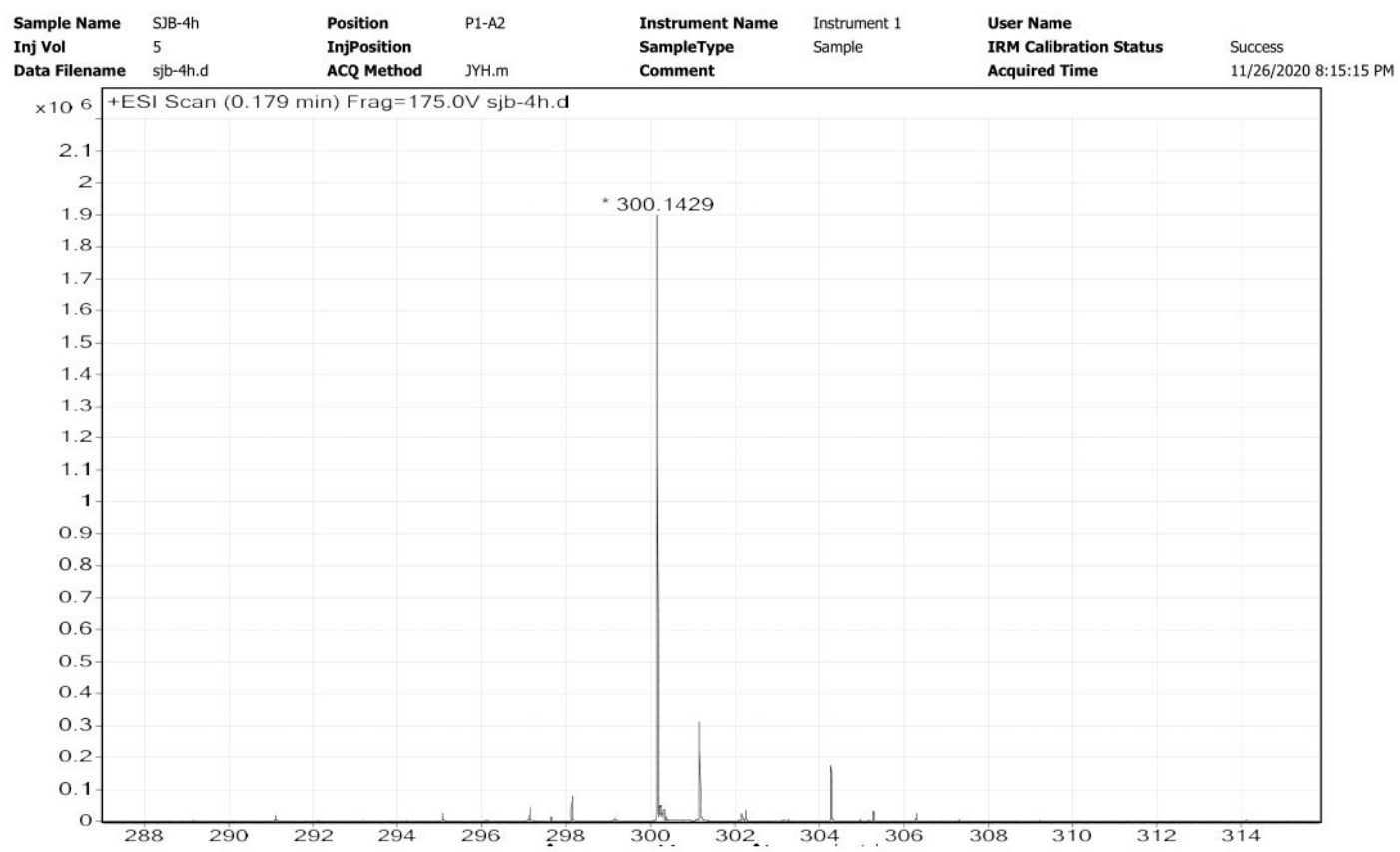

\begin{tabular}{|c|r|r|r|r|r|r|r|}
\hline Formula (M) & Score (MFG) & Mass & Mass (MFG) & m/z (Calc) & Diff (ppm) & DBE & $\mathrm{m} / \mathrm{z}$ \\
\hline C13 H19 N5 O2 & 99.86 & 277.1537 & 277.1539 & 300.1431 & 0.71 & 7 & 300.1429 \\
\hline
\end{tabular}<smiles>COc1ccc(N=NC(CN)CO)cc1</smiles>

$4 i$

HRMS (ESI+): $[\mathrm{M}+\mathrm{Na}]^{+}$Calculated for $\mathrm{C}_{17} \mathrm{H}_{27} \mathrm{~N}_{5} \mathrm{O}_{2} \mathrm{Na}$ : 356.2057, Found 356.2056.

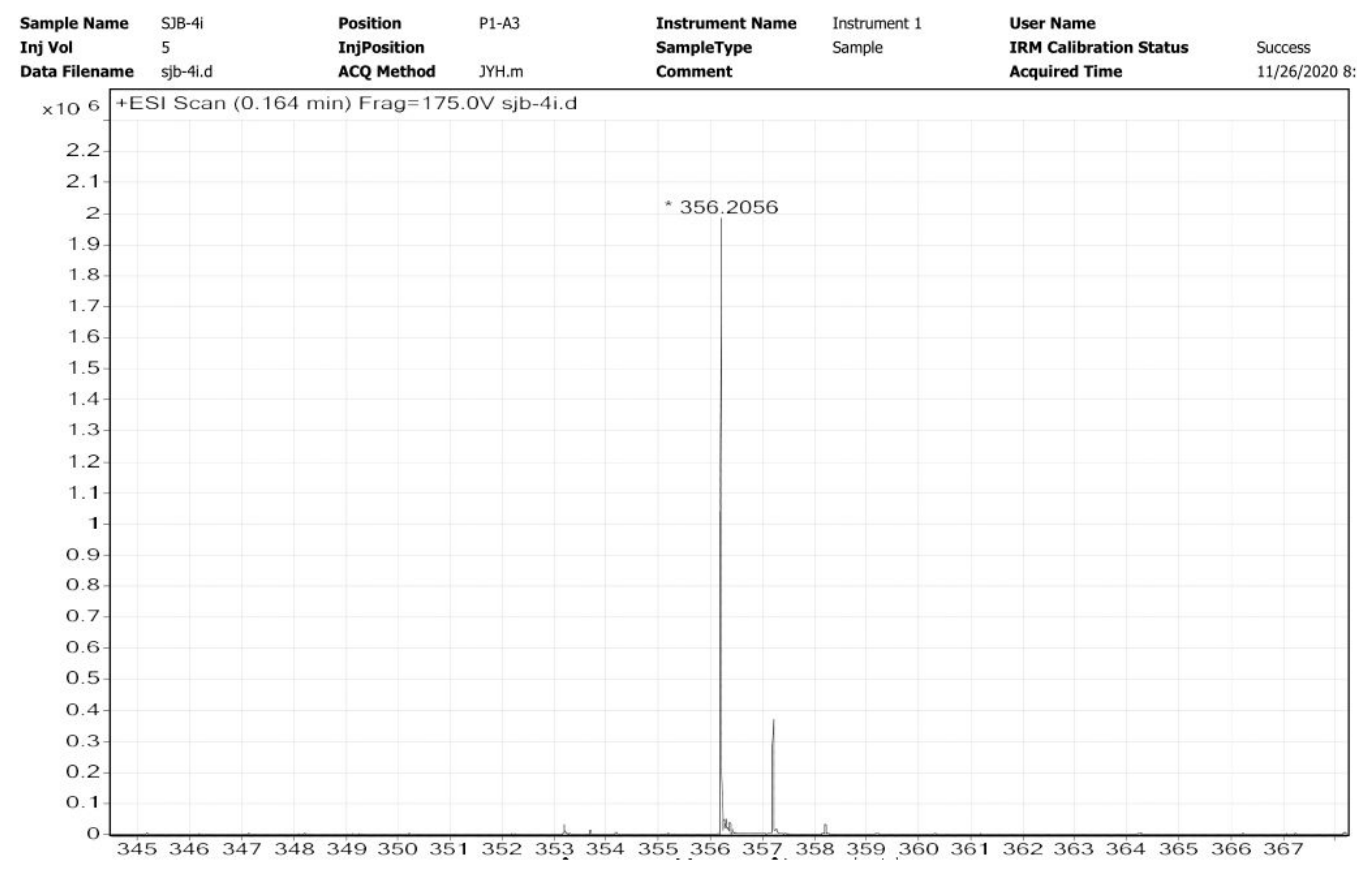

\begin{tabular}{|c|r|r|r|r|r|r|r|}
\hline Formula (M) & Score (MFG) & Mass & Mass (MFG) & $\mathrm{m} / \mathrm{z}($ Calc) & Diff (ppm) & DBE & $\mathrm{m} / \mathrm{z}$ \\
\hline C17 H27 N5 O2 & 99.97 & 333.2164 & 333.2165 & 356.2057 & 0.29 & 3 & 356.2056 \\
\hline
\end{tabular}




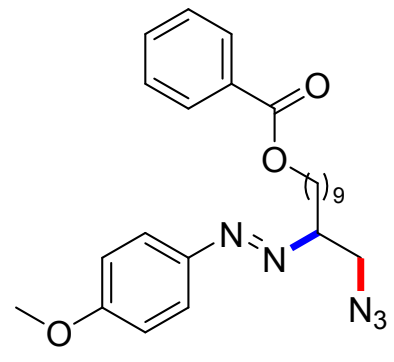

$4 n$

HRMS (ESI): $[\mathrm{M}+\mathrm{Na}]^{+}$Calculated for $\mathrm{C}_{25} \mathrm{H}_{33} \mathrm{~N}_{5} \mathrm{O}_{3} \mathrm{Na}$ : 474.2476, Found 474.2477.

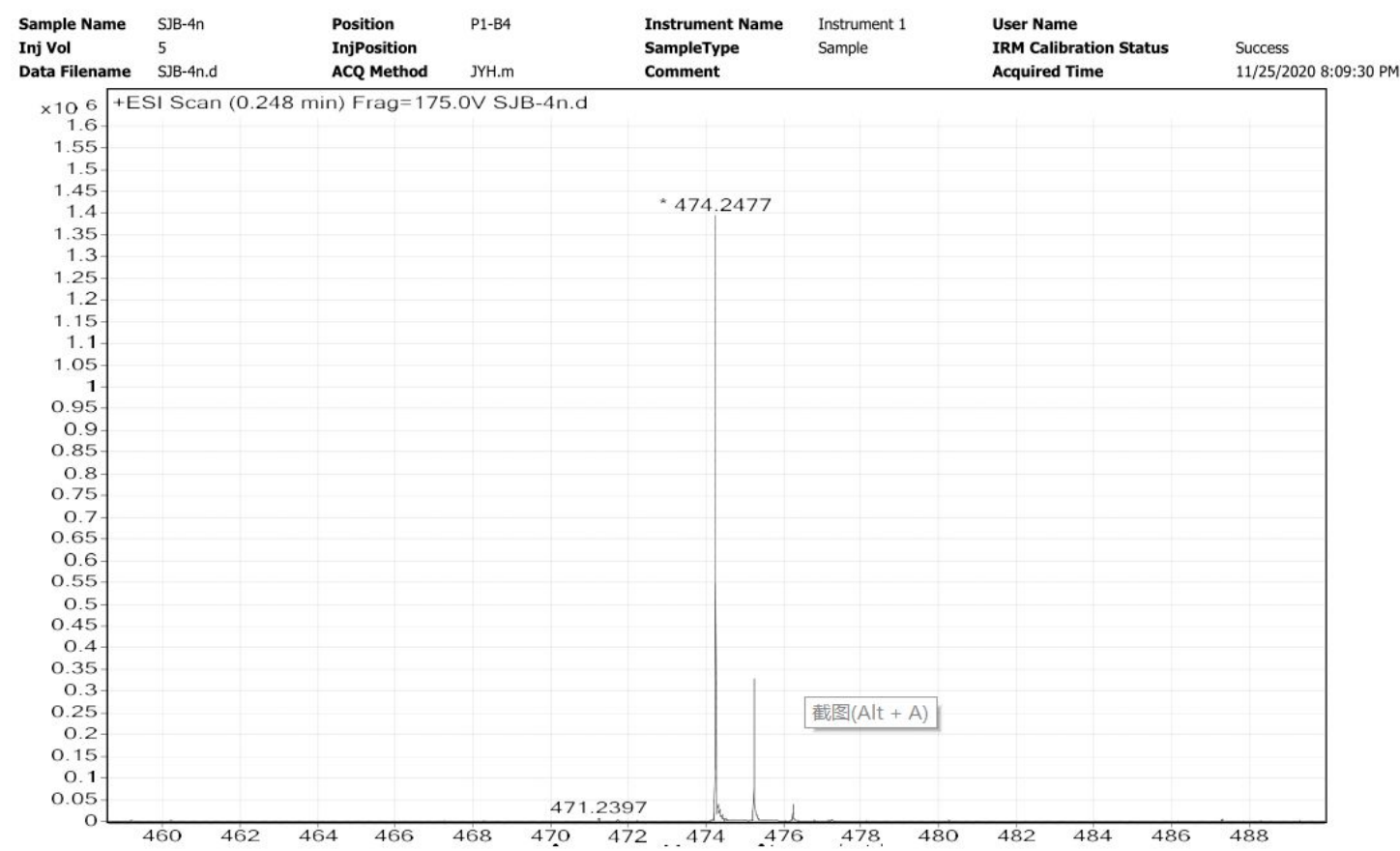

\begin{tabular}{|c|r|r|r|r|r|r|c|}
\hline Formula (M) & Score (MFG) & \multicolumn{1}{c|}{ Mass } & Mass (MFG) & $\mathrm{m} / \mathrm{z}$ (Calc) & Diff (ppm) & DBE & $\mathrm{m} / \mathrm{z}$ \\
\hline C25 H33 N5 O3 & 99.96 & 451.2585 & 451.2583 & 474.2476 & -0.31 & 12 & 474.2477 \\
\hline
\end{tabular}

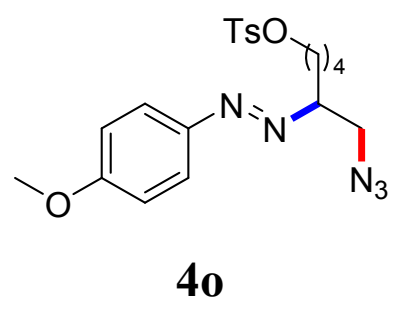

HRMS (ESI): $[\mathrm{M}+\mathrm{Na}]^{+}$Calculated for $\mathrm{C}_{20} \mathrm{H}_{25} \mathrm{~N}_{5} \mathrm{O}_{4} \mathrm{SNa}$ : 454.1519, Found 454.1518 . 


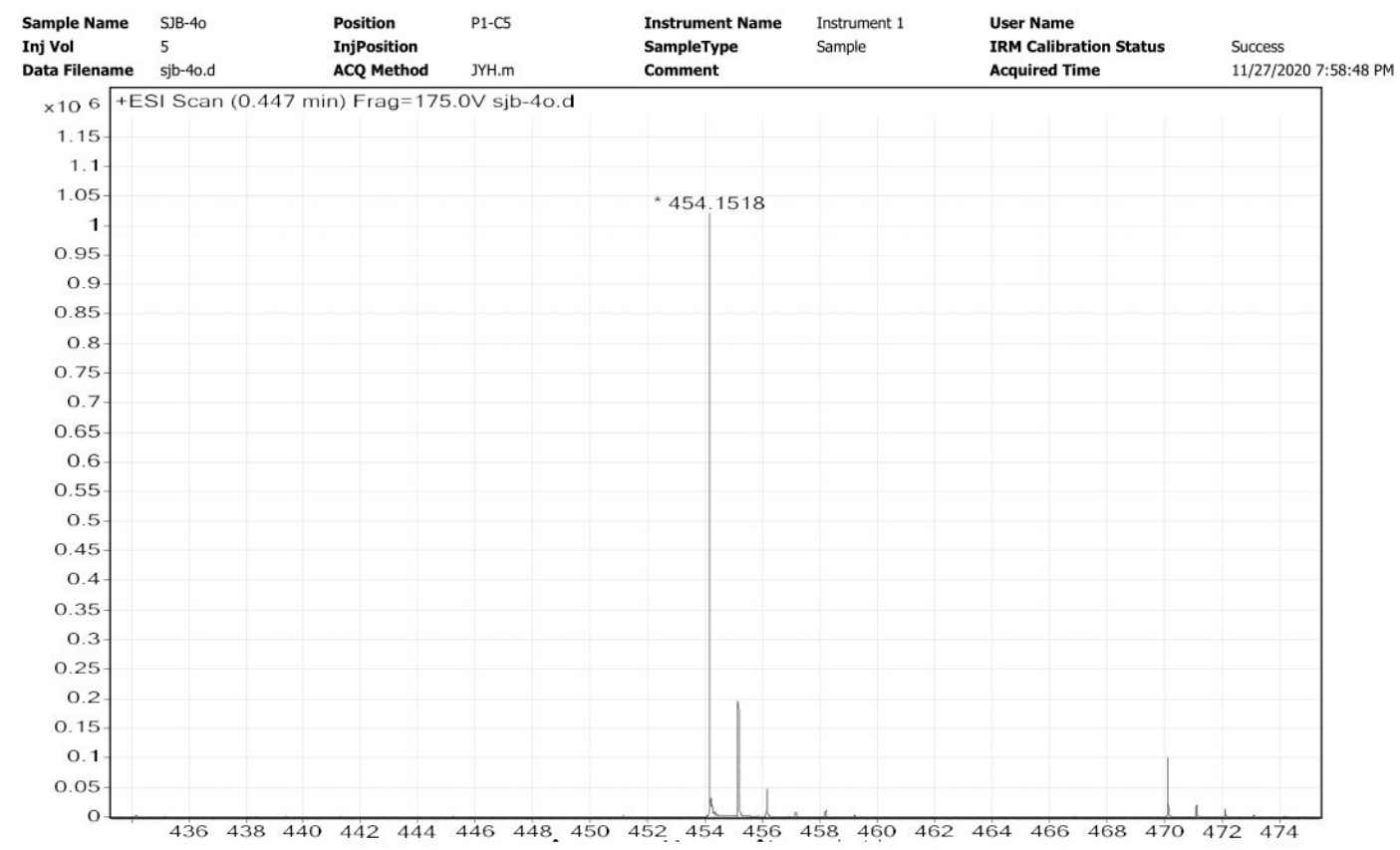

\begin{tabular}{|l|r|r|r|r|r|r|r|}
\hline Formula (M) & Score (MFG) & \multicolumn{1}{c|}{ Mass } & Mass (MFG) & $\mathrm{m} / \mathrm{z}$ (Calc) & Diff (ppm) & DBE & $\mathrm{m} / \mathrm{z}$ \\
\hline C20 H25 N5 O4 S & 99.95 & 431.1626 & 431.1627 & 454.1519 & 0.34 & & 11 \\
\hline
\end{tabular}<smiles>CCCC(CN)N=Nc1ccc(C)cc1</smiles>

\section{$4 \mathbf{t}$}

HRMS (ESI): $[\mathrm{M}+\mathrm{Na}]^{+}$Calculated for $\mathrm{C}_{12} \mathrm{H}_{17} \mathrm{~N}_{5} \mathrm{Na}$ : 254.1376, Found 254.1378.

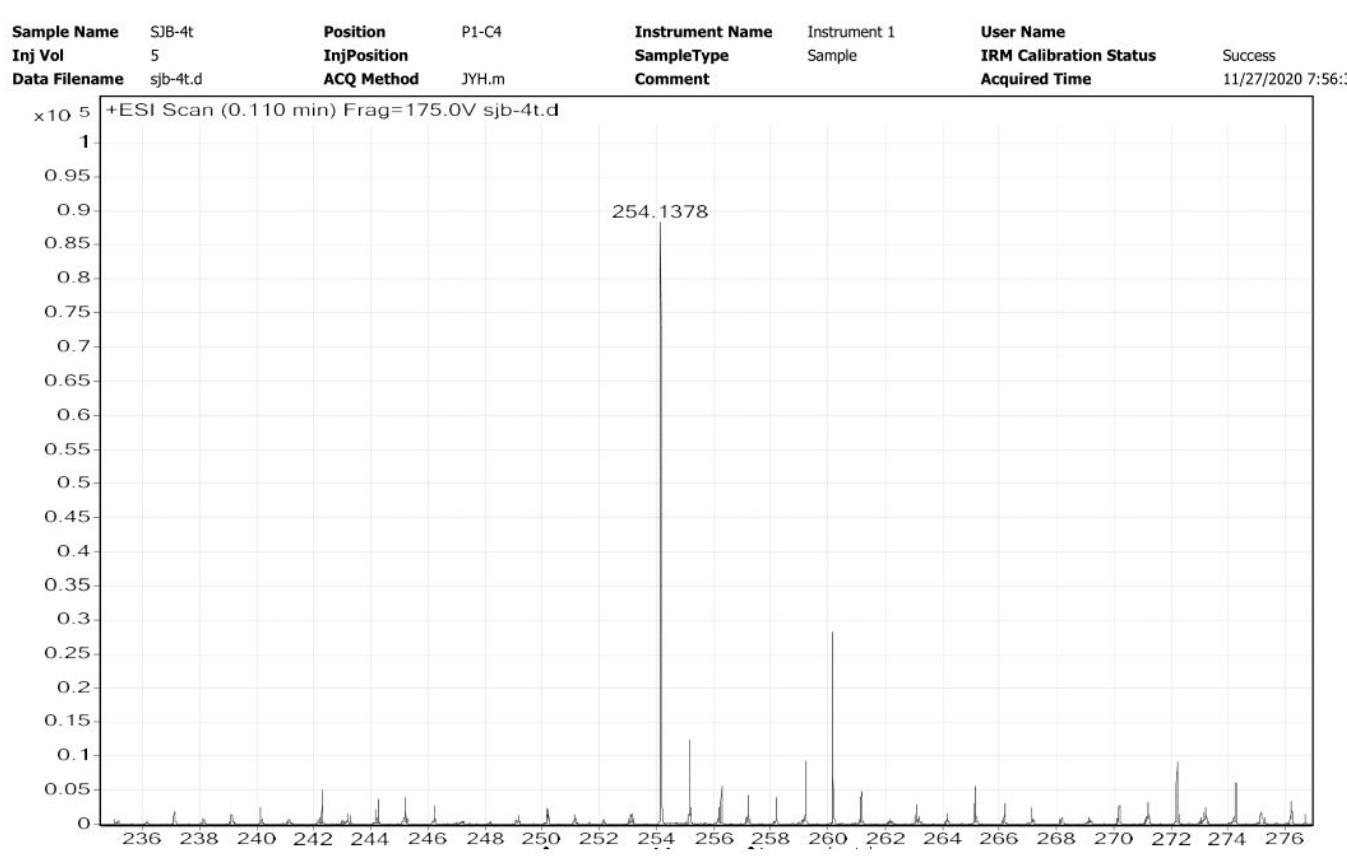

\begin{tabular}{|r|r|r|r|r|r|r|r|}
\hline Formula (M) & Score (MFG) & Mass & Mass (MFG) & $\mathrm{m} / \mathrm{z}$ (Calc) & Diff (ppm) & DBE & $\mathrm{m} / \mathrm{z}$ \\
\hline $\mathrm{C} 12 \mathrm{H} 17 \mathrm{N5}$ & 99.85 & 231.1486 & 231.1484 & 254.1376 & -0.79 & 7 & 254.1378 \\
\hline
\end{tabular}


<smiles>CCCC(CN)N=Nc1ccc(C#N)cc1</smiles>

$4 u$

HRMS (ESI): [M+Na] ${ }^{+}$Calculated for $\mathrm{C}_{12} \mathrm{H}_{14} \mathrm{~N}_{6} \mathrm{Na}$ : 265.1172, Found 265.1174.

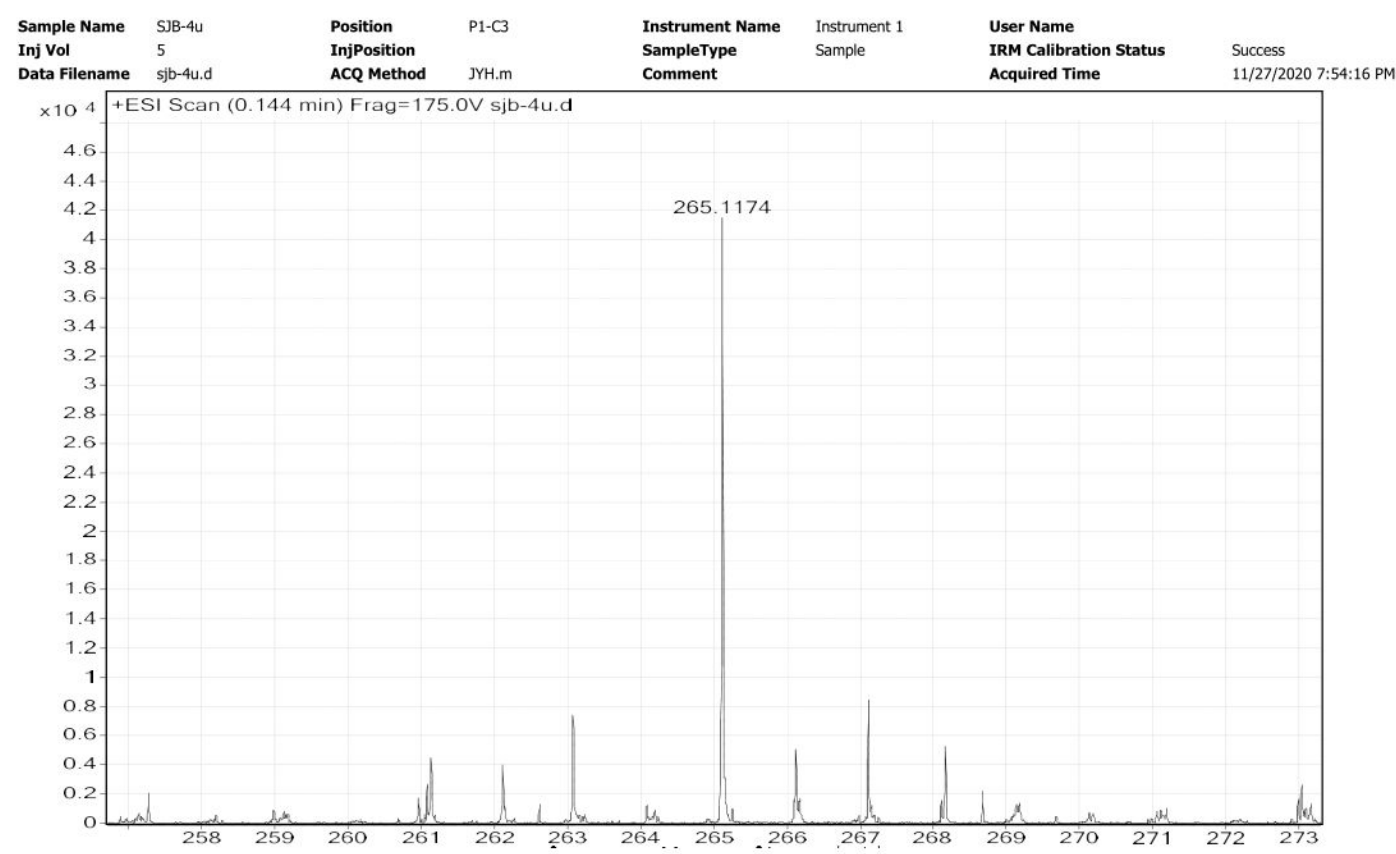

\begin{tabular}{|r|r|r|r|r|r|r|r|}
\hline Formula (M) & Score (MFG) & Mass & Mass (MFG) & $\mathrm{m} / \mathrm{z}($ Calc) & Diff (ppm) & DBE & $\mathrm{m} / \mathrm{z}$ \\
\hline C12 H14 N6 & 99.86 & 242.1282 & 242.128 & 265.1172 & -0.76 & 9 & 265.1174 \\
\hline
\end{tabular}<smiles>Cc1cccc(N=NC(CN)C(C)C)c1</smiles>

$4 x$

HRMS (ESI): [M+Na] $]^{+}$Calculated for $\mathrm{C}_{12} \mathrm{H}_{17} \mathrm{~N}_{5} \mathrm{Na}$ : 254.1376, Found 254.1378. 


\begin{tabular}{|c|c|c|c|c|c|c|c|}
\hline $\begin{array}{l}\text { Sample Name } \\
\text { Inj Vol }\end{array}$ & $\begin{array}{l}\text { SJB-4w } \\
5\end{array}$ & $\begin{array}{l}\text { Position } \\
\text { InjPosition }\end{array}$ & P1-B4 & $\begin{array}{l}\text { Instrument Name } \\
\text { SampleType }\end{array}$ & $\begin{array}{l}\text { Instrument } 1 \\
\text { Sample }\end{array}$ & $\begin{array}{l}\text { User Name } \\
\text { IRM Calibration Status }\end{array}$ & Success \\
\hline Data Filename & SJB-4w.d & ACQ Method & JYH.m & Comment & & Acquired Time & 12/1/2020 7:16:43 PM \\
\hline
\end{tabular}

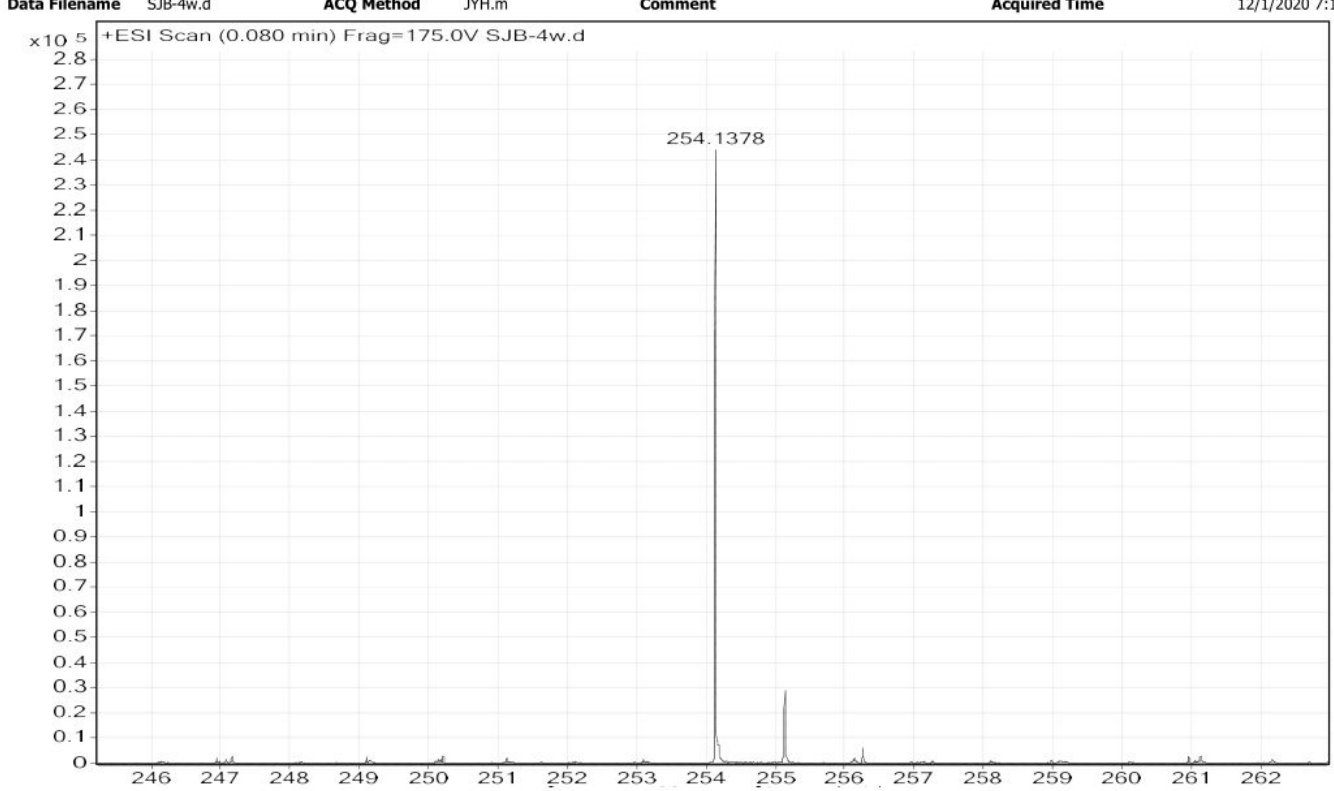

\begin{tabular}{|r|r|r|r|r|r|r|r|}
\hline Formula (M) & Score (MFG) & \multicolumn{1}{c|}{ Mass } & Mass (MFG) & \multicolumn{1}{c|}{$\mathrm{m} / \mathrm{z}$ (Calc) } & Diff (ppm) & DBE & $\mathrm{m} / \mathrm{z}$ \\
\hline C12 H17 N5 & 99.85 & 231.1486 & 231.1484 & 254.1376 & -0.79 & & 254.1378 \\
\hline
\end{tabular}<smiles>CCCC(CN)N=Nc1cccc(F)c1</smiles>

\section{$4 y$}

HRMS (ESI): [M+Na] $]^{+}$Calculated for $\mathrm{C}_{11} \mathrm{H}_{14} \mathrm{FN}_{5} \mathrm{Na}$ : 258.1125, Found 258.1126.

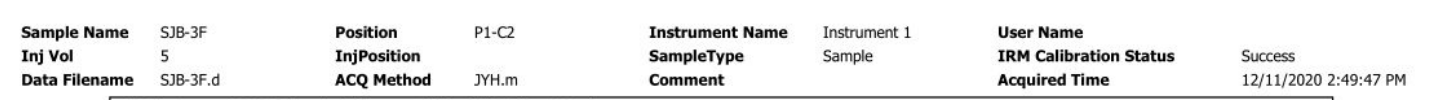

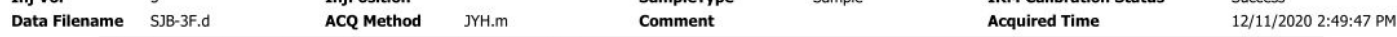

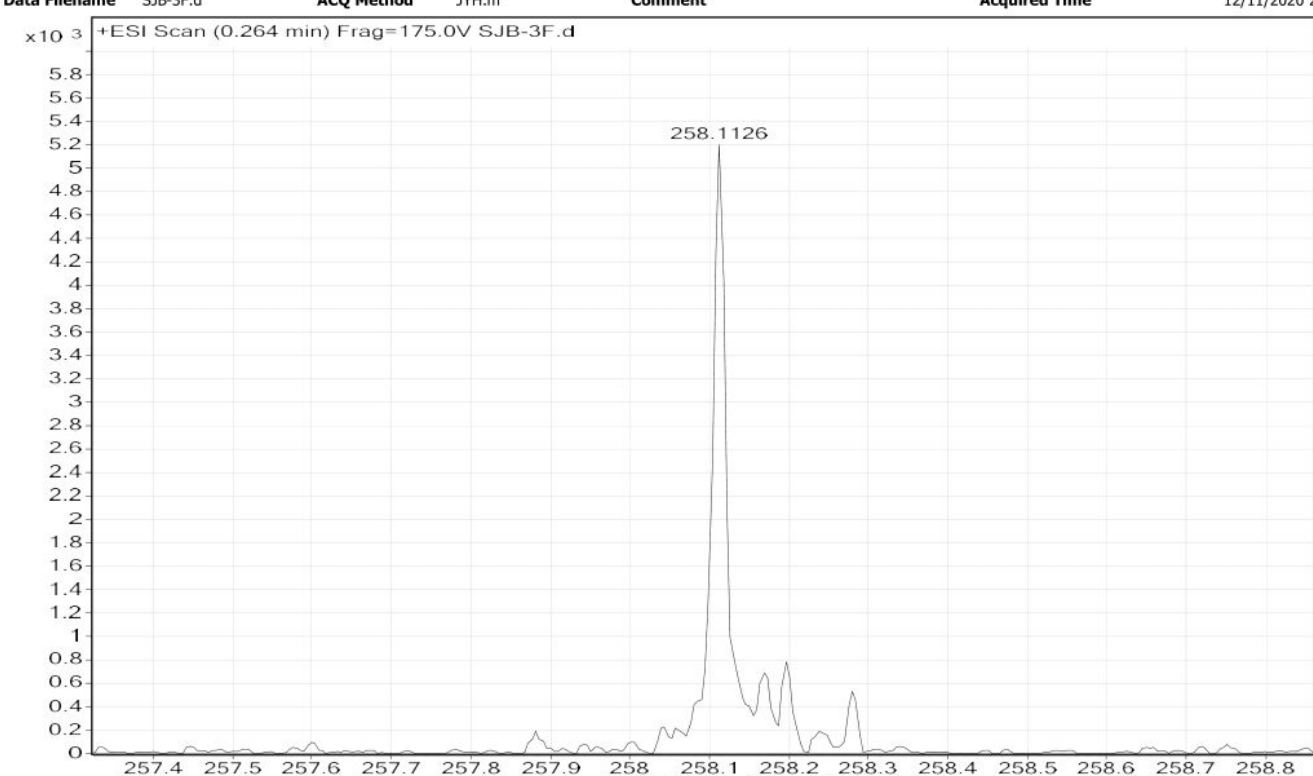

\begin{tabular}{r|r|r|r|r|r|r|r} 
Formula (M) & Score (MFG) & \multicolumn{1}{c|}{ Mass } & Mass (MFG) & $\mathrm{m} / \mathrm{z}$ (Calc) & Diff (ppm) & DBE & $\mathrm{m} / \mathrm{z}$ \\
\hline C11 H14 F N5 & 99.99 & 235.1234 & 235.1233 & 258.1125 & -0.23 & 7 & 258.1126 \\
\hline
\end{tabular}


<smiles>CC(C)C(CN)N=Nc1cccc2ccccc12</smiles>

\section{4ad}

HRMS (ESI): [M+Na] $]^{+}$Calculated for $\mathrm{C}_{15} \mathrm{H}_{17} \mathrm{~N}_{5} \mathrm{Na}$ : 290.1376, Found 290.1374 .

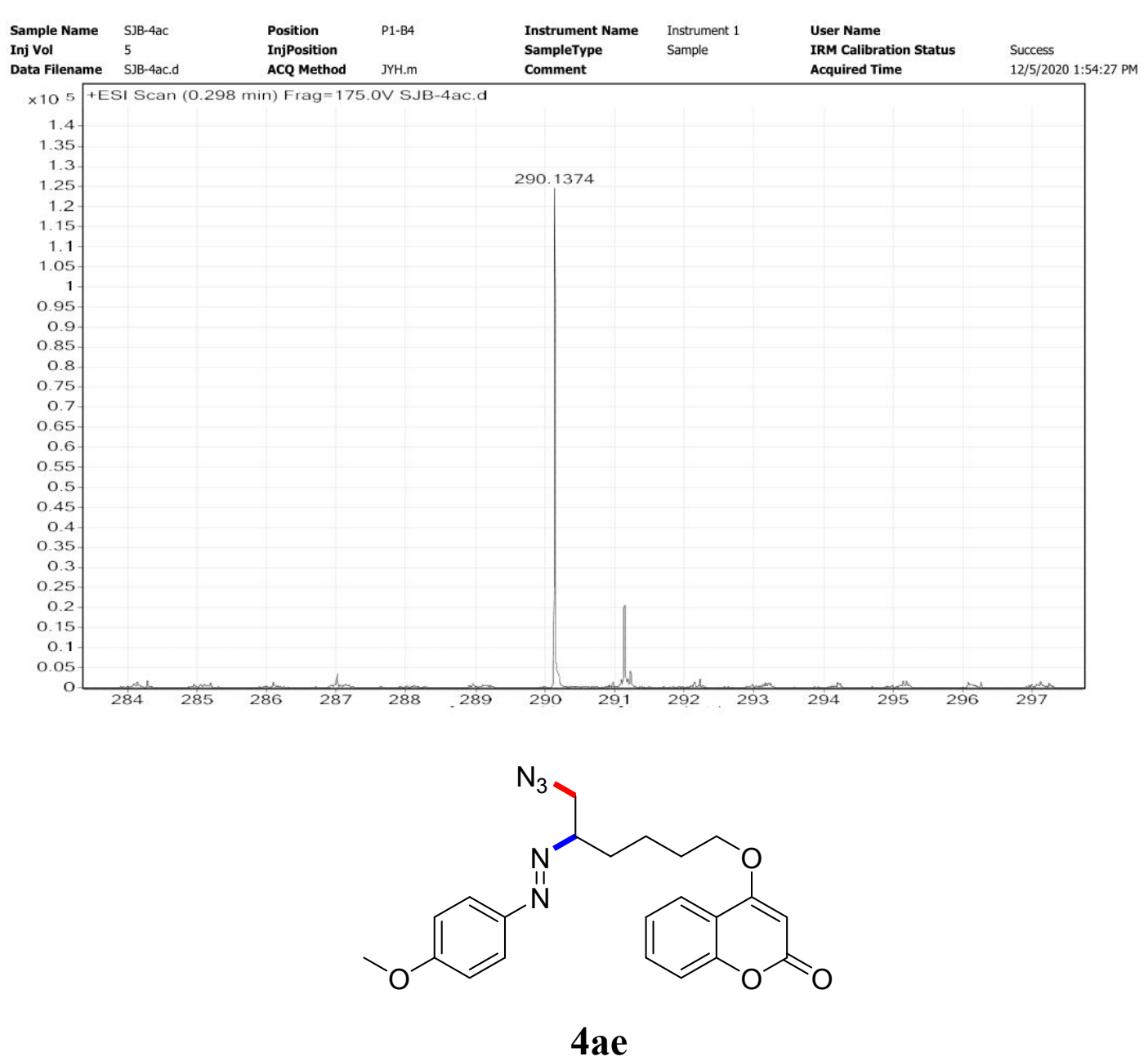

HRMS (ESI): $[\mathrm{M}+\mathrm{Na}]^{+}$Calculated for $\mathrm{C}_{22} \mathrm{H}_{23} \mathrm{~N}_{5} \mathrm{O}_{4} \mathrm{Na}: 444.1642$, Found 444.1633. 

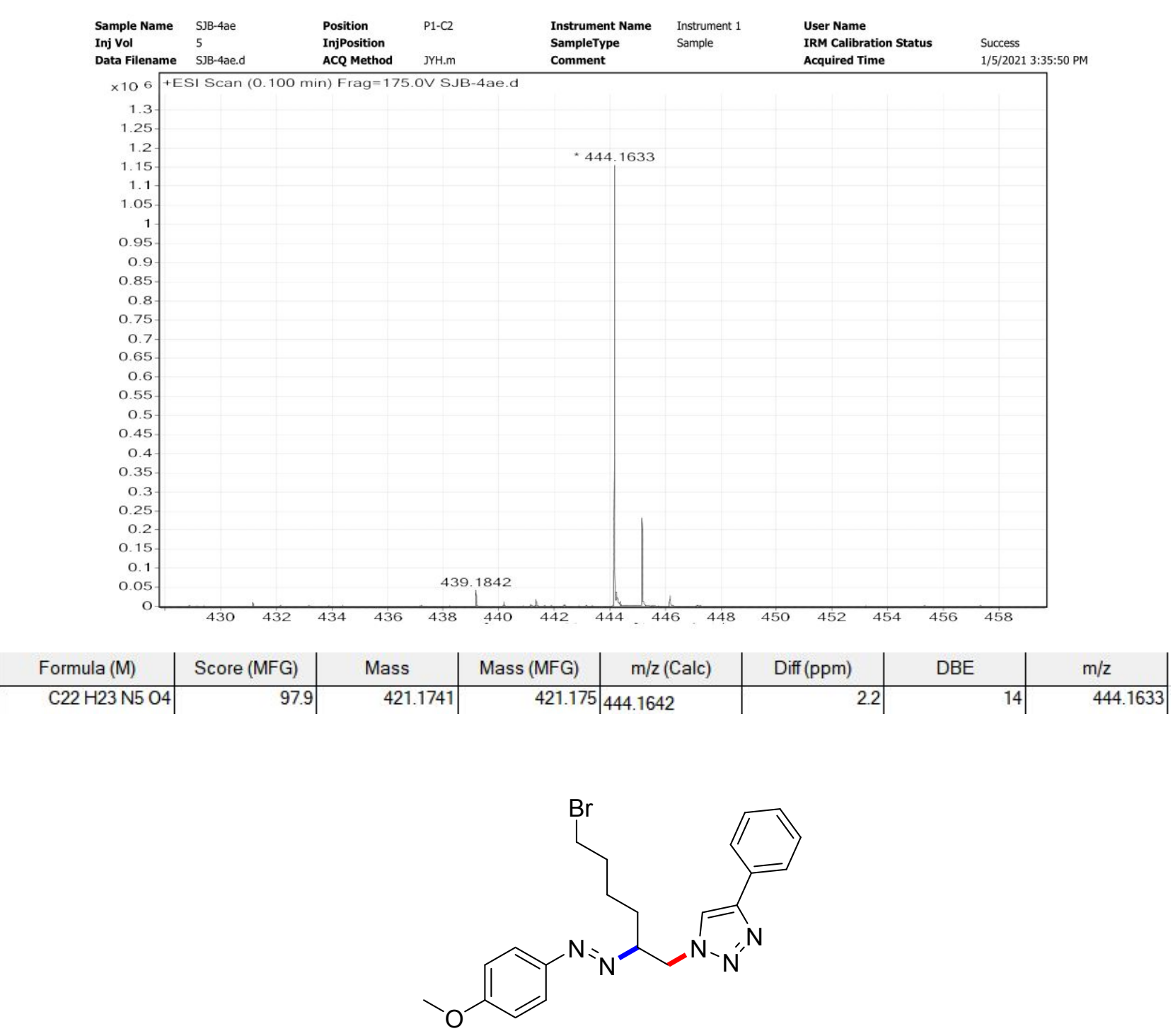

4af

HRMS (ESI): $[\mathrm{M}+\mathrm{Na}]^{+}$Calculated for $\mathrm{C}_{21} \mathrm{H}_{24} \mathrm{BrN}_{5} \mathrm{ONa}$ : 464.1056, Found 464.1051.

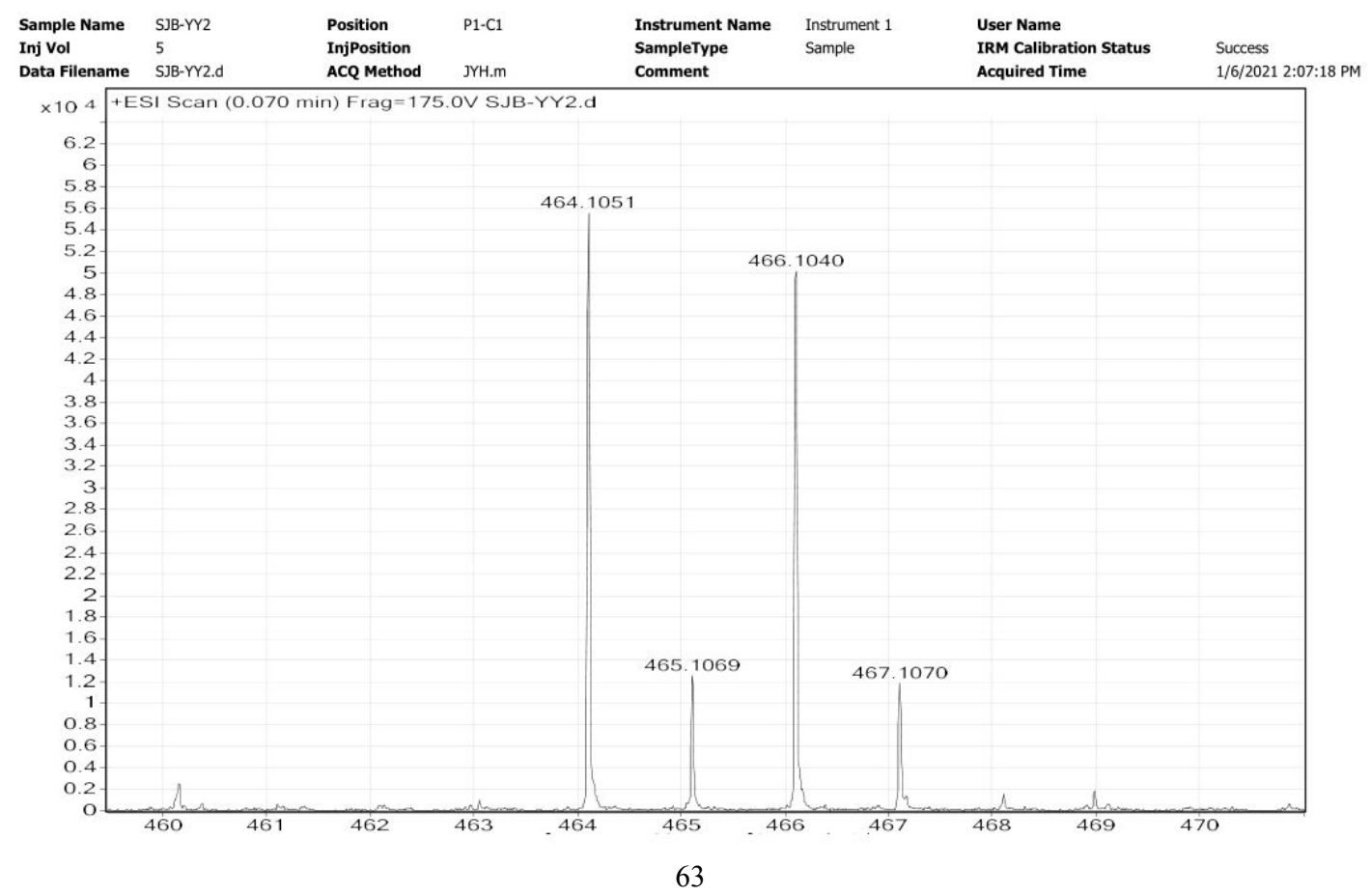




\begin{tabular}{|l|r|r|r|r|r|r|c|} 
Formula (M) & Score (MFG) & \multicolumn{1}{c|}{ Mass } & Mass (MFG) & \multicolumn{1}{c|}{$\mathrm{m} / \mathrm{z}$ (Calc) } & Diff (ppm) & \multicolumn{1}{c|}{ DBE } & $\mathrm{m} / \mathrm{z}$ \\
\hline C21 H24 Br N5 O & 99.3 & 441.1159 & 441.1164 & 464.1056 & 1.23 & 12464.1051
\end{tabular}<smiles>COc1ccc(N=NC(C)(C)C([NH3+])CC[C@H](C)CCO)cc1</smiles>

\section{4ah}

HRMS (ESI): $[\mathrm{M}+\mathrm{Na}]^{+}$Calculated for $\mathrm{C}_{17} \mathrm{H}_{27} \mathrm{~N}_{5} \mathrm{O}_{2} \mathrm{Na}$ : 356.2057, Found 356.2060.

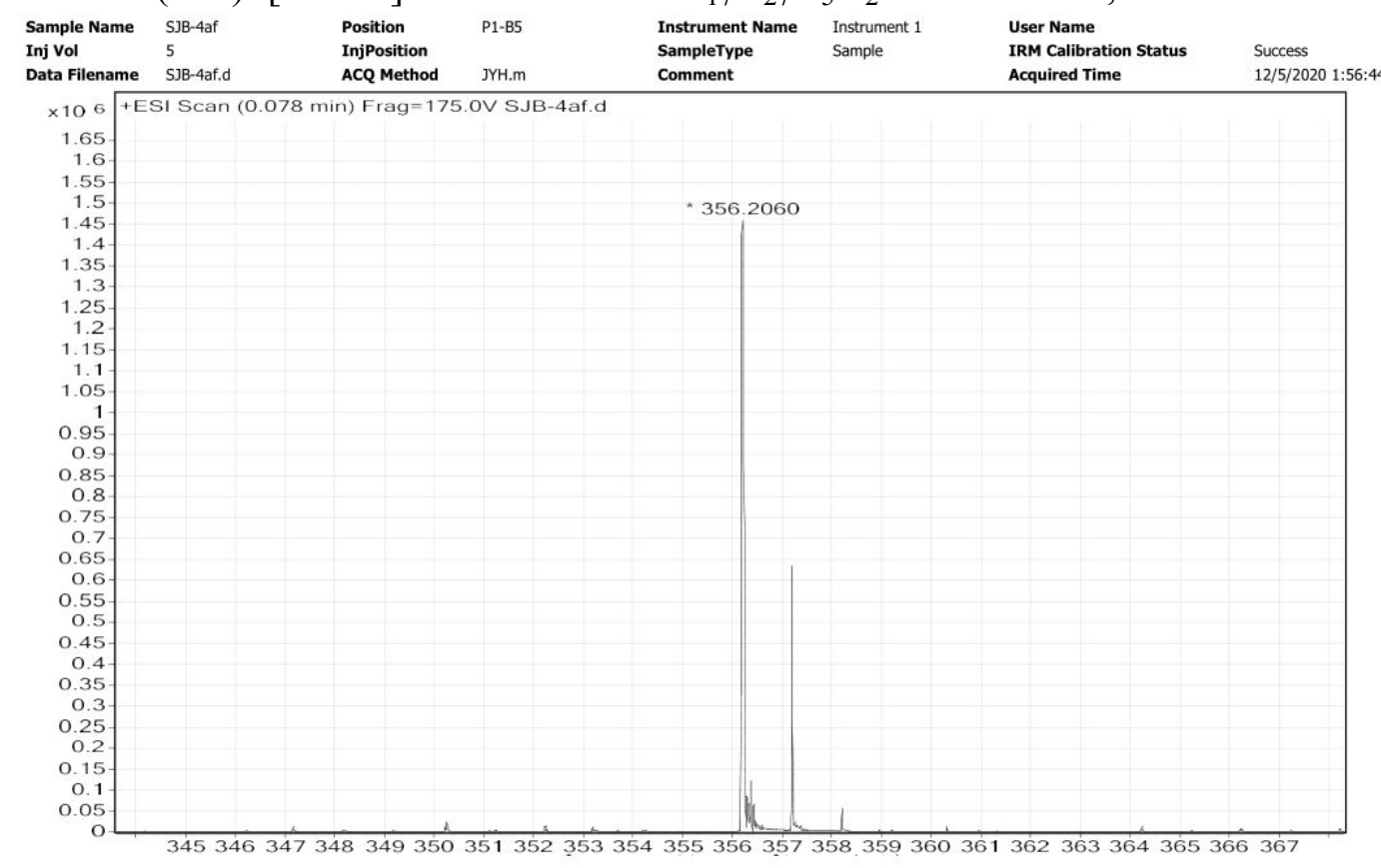

\begin{tabular}{|c|r|r|r|r|r|r|r|}
\hline Formula (M) & \multicolumn{1}{|c|}{ Score (MFG) } & \multicolumn{1}{c|}{ Mass } & Mass (MFG) & \multicolumn{1}{|c|}{$\mathrm{m} / \mathrm{z}$ (Calc) } & Diff (ppm) & \multicolumn{1}{c|}{ DBE } & \multicolumn{1}{c|}{$\mathrm{m} / \mathrm{z}$} \\
\hline C17 H27 N5 02 & 99.71 & 333.2168 & 333.2165 & 356.2057 & -0.91 & 7 & 356.206 \\
\hline
\end{tabular}<smiles>COc1ccc(N=NC(C)(CN)C2CCC3=CC(=O)C[C@H](C)[C@H]3C2)cc1</smiles>

4ai

HRMS (ESI): $[\mathrm{M}+\mathrm{Na}]^{+}$Calculated for $\mathrm{C}_{22} \mathrm{H}_{29} \mathrm{~N}_{5} \mathrm{O}_{2} \mathrm{Na}$ : 418.2213, Found 418.2222. 


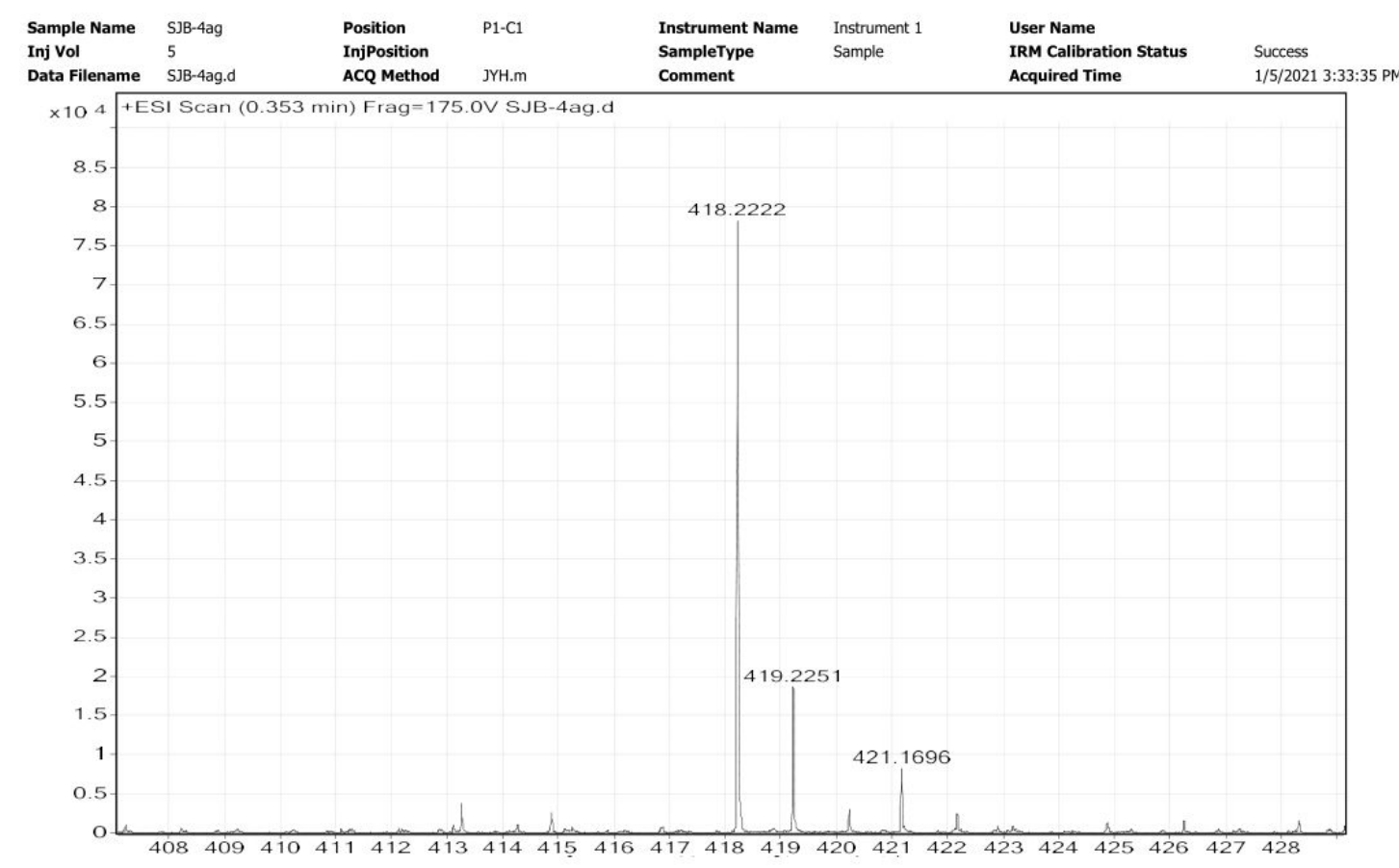

\begin{tabular}{|c|r|r|r|r|r|r|r|r} 
Formula (M) & Score (MFG) & Mass & Mass (MFG) & $\mathrm{m} / \mathrm{z}$ (Calc) & Diff (ppm) & DBE & $\mathrm{m} / \mathrm{z}$ \\
\hline $\mathrm{C} 22 \mathrm{H} 29 \mathrm{~N} 5 \mathrm{O} 2$ & 98.08 & 395.233 & 395.2321 & 418.2213 & -2.16 & 418.2222 \\
\hline
\end{tabular}<smiles>CCCC(CN)ON1C(C)(C)CCCC1(C)C</smiles>

HRMS (ESI): [M+Na] $]^{+}$Calculated for $\mathrm{C}_{14} \mathrm{H}_{28} \mathrm{~N}_{4} \mathrm{ONa}$ : 269.2336, Found 269.2329.

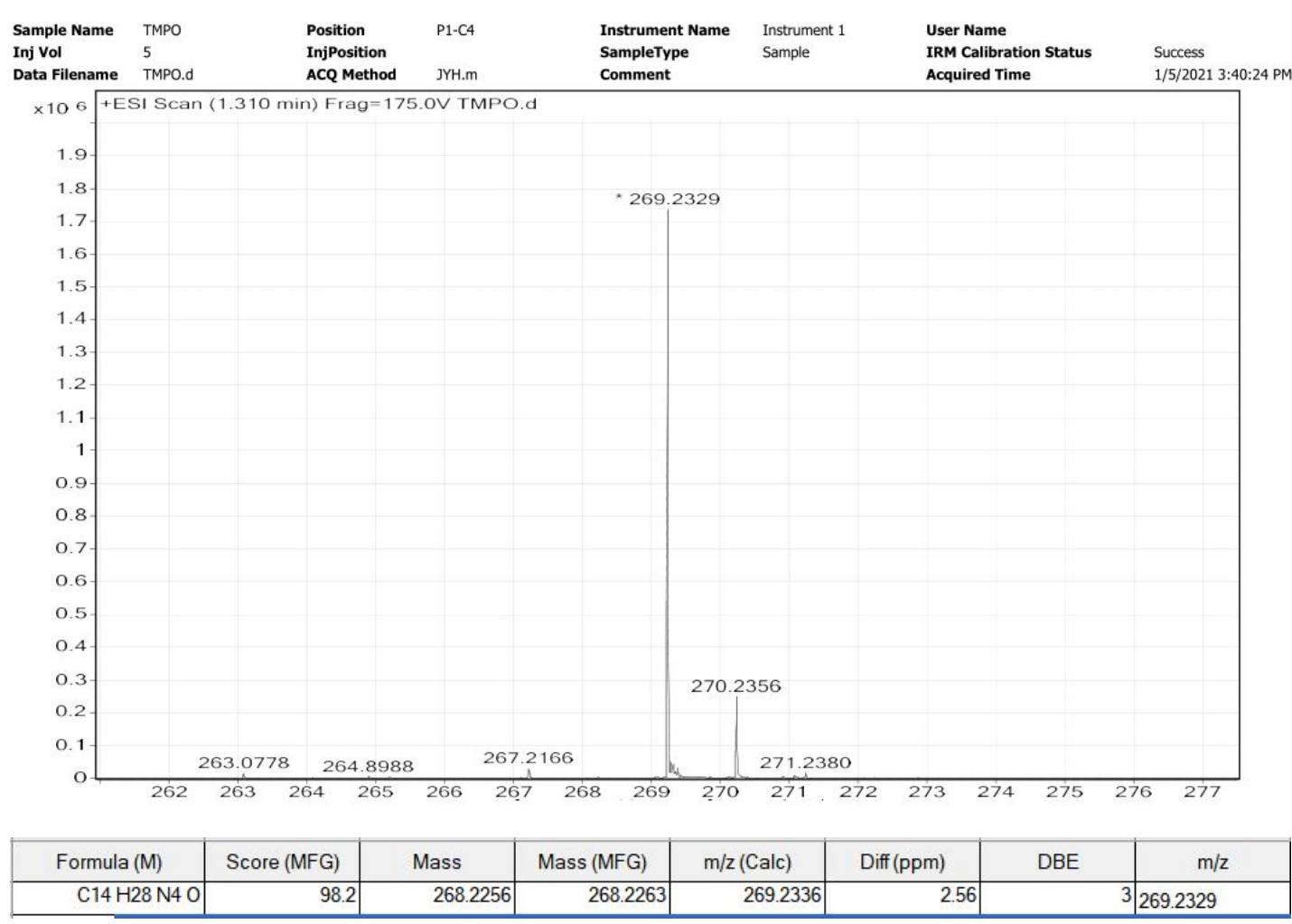




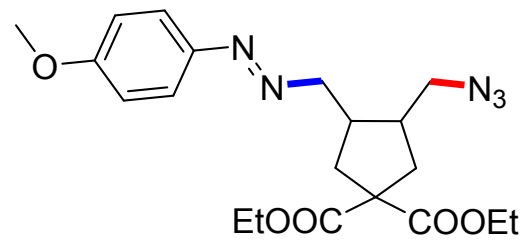

4aj

HRMS (ESI): $[\mathrm{M}+\mathrm{Na}]^{+}$Calculated for $\mathrm{C}_{20} \mathrm{H}_{27} \mathrm{~N}_{5} \mathrm{O}_{5} \mathrm{Na}$ : 440.1904, Found 440.1901 .

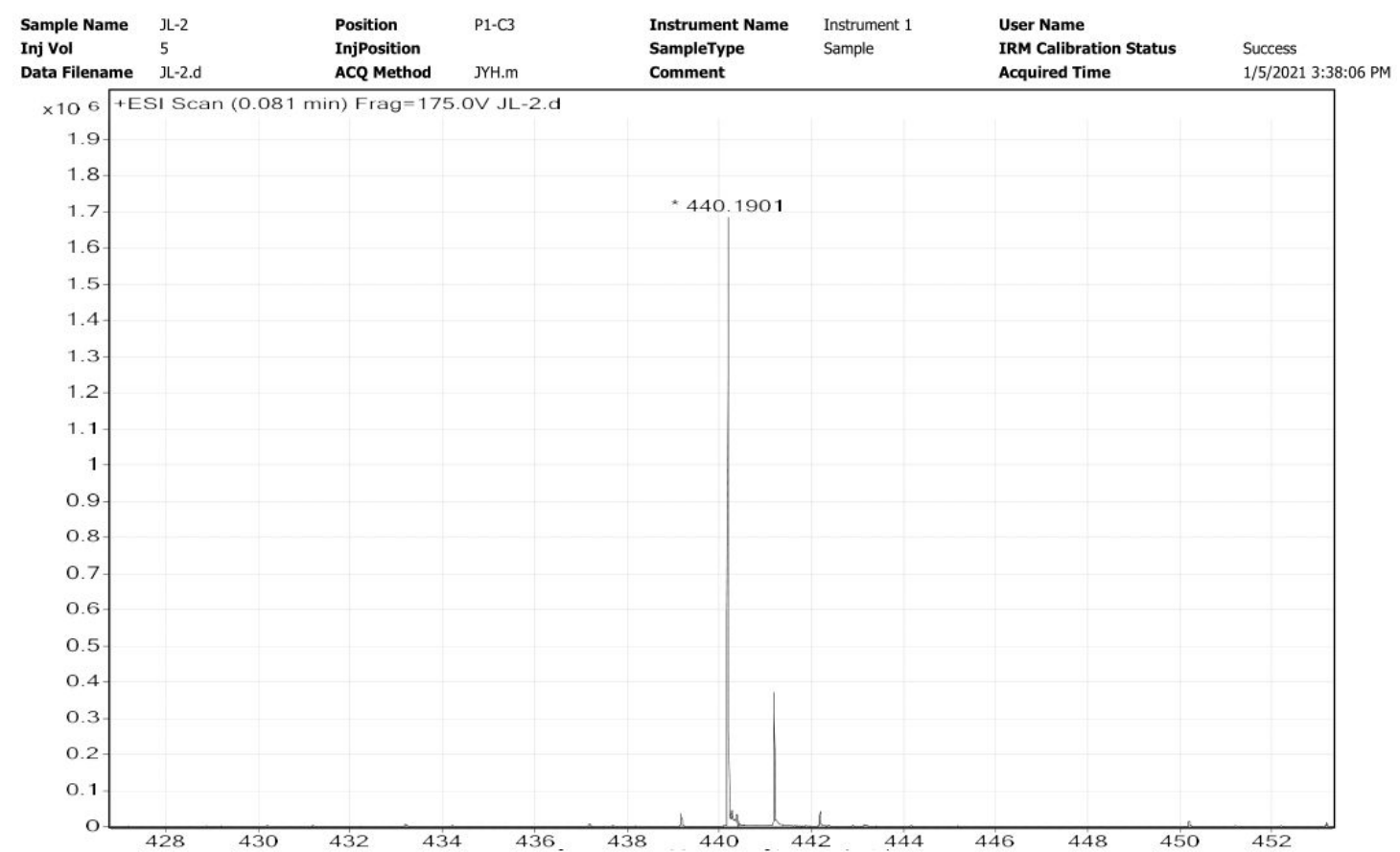

\begin{tabular}{c|r|r|r|r|r|r|c|}
\hline Formula (M) & Score (MFG) & \multicolumn{1}{c|}{ Mass } & Mass (MFG) & $\mathrm{m} / \mathrm{z}$ (Calc) & Diff (ppm) & DBE & $\mathrm{m} / \mathrm{z}$ \\
\hline C20 H27 N5 05 & 99.71 & 417.2009 & 417.2012 & 440.1904 & 0.82 & 10 & 440.1901 \\
\hline
\end{tabular}

